\title{
Portable transparent indenter instrumentation for material surface characterization
}

Julio A. Noriega

West Virginia University

Follow this and additional works at: https://researchrepository.wvu.edu/etd

\section{Recommended Citation}

Noriega, Julio A., "Portable transparent indenter instrumentation for material surface characterization" (2006). Graduate Theses, Dissertations, and Problem Reports. 4251.

https://researchrepository.wvu.edu/etd/4251

This Dissertation is protected by copyright and/or related rights. It has been brought to you by the The Research Repository @ WVU with permission from the rights-holder(s). You are free to use this Dissertation in any way that is permitted by the copyright and related rights legislation that applies to your use. For other uses you must obtain permission from the rights-holder(s) directly, unless additional rights are indicated by a Creative Commons license in the record and/ or on the work itself. This Dissertation has been accepted for inclusion in WVU Graduate Theses, Dissertations, and Problem Reports collection by an authorized administrator of The Research Repository @ WVU.

For more information, please contact researchrepository@mail.wvu.edu. 


\title{
Portable Transparent Indenter Instrumentation for Material Surface Characterization
}

\author{
Julio A. Noriega \\ Dissertation submitted to the College of Engineering and Mineral Resources \\ at West Virginia University \\ in partial fulfillment of the requirements \\ for the degree of
}

\section{Doctor of Philosophy}

In

Mechanical Engineering

\author{
Bruce S. Kang, Ph.D., Chair \\ Ever Barbero, Ph.D. \\ Jacky Prucz, Ph.D. \\ Xingbo Liu, Ph.D. \\ Christopher Johnson, Ph.D. \\ Department of Mechanical and Aerospace Engineering \\ Morgantown, West Virginia \\ 2006
}

Keywords: Transparent Indenter Measurement, Stress-Strain curve, Indentation Stress-Strain curve, Hardness, Instrumented Indentation Testing. 


\section{ABSTRACT \\ Portable Transparent Indenter Instrumentation for Material Surface Characterization}

A portable Transparent Indenter Measurement (TIM) instrument that combines Instrumented Indentation Testing characteristics with optical microscopy through a transparent half ball indenter has been developed. The TIM instrument can provide the following experimental data: a) the load applied as the indenter penetrates the specimen, b) the displacement of the piezoelectric tip, and c) the indented area. All these data are used to obtain the material stress-strain curve, through the determination of the elastic modulus, yield strength, and strain hardening exponent.

Using the TIM instrument and based on Hertz elastic contact theory, a new method to obtain material elastic modulus is developed. A second approach to calculate the material elastic modulus by the use of TIM Instrumented Indentation and the method proposed by Oliver and Pharr is also presented. 
To my family. My wife, Maria Natalia, and my children, Anamaria Rosalina, Julio Raul and Nathalie Lucia. Thanks very much for your love, support and sacrifice. 


\section{ACKNOWLEDGEMENTS}

I would like to thank my committee members Dr. Christopher Johnson, Dr. Xingbo Liu, Dr. Jacky Prucz, and Dr. Ever Barbero. I would like to thank especially my advisor Dr. Bruce S. Kang, for his support and guidance during the past years. It has been a pleasure working with and learning from him. I would like to thank all the members in Dr. Kang's research group, Wayne Lee, John Sakacsi, Erin Lin and Ashook Varadarajan. A special thank to Dr. Chuanyu Feng for his constructive suggestion and idea in the development of this dissertation.

Thanks to my friends Eduardo Perez, Charlotte Arana, Omar Meza, Marlene Dearteano, Francisco Elizalde, Denny Catacora, Hermann Alcazar, Francisco Pino, and Jacinto Solano. I must give special thanks to Dr. Eduardo Sosa and Alex Tsai who read and helped me in the edition of the manuscript.

Finally, I must give my thanks to my family. To my wife Natalia Ovalle. Her love and sacrifice in supporting our home during these three years. To Anamaria, Julio and Nathalie, for their love and sacrifice while I was far from home. 


\section{TABLE OF CONTENTS}

TITLE PAGE

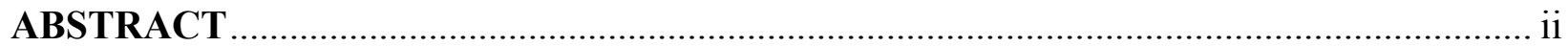

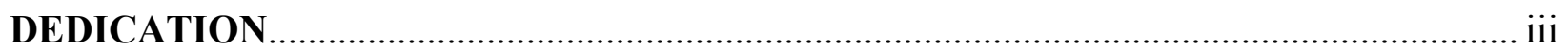

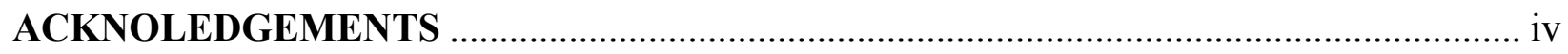

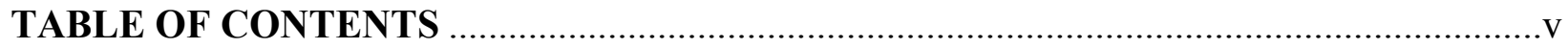

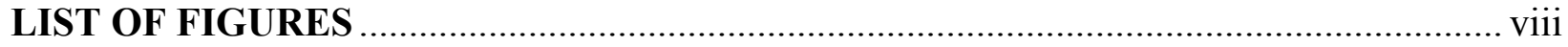

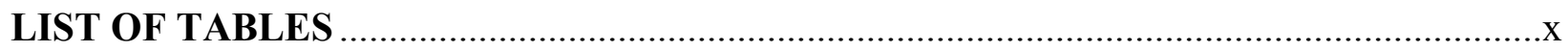

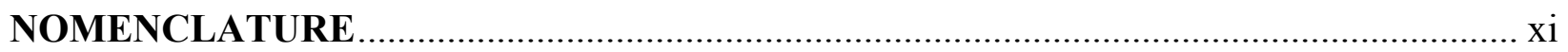

Chapter 1 INTRODUCTION AND RESEARCH OBJECTIVE ......................................... 1

Chapter 2 LITERATURE REVIEW …........................................................................................ 4

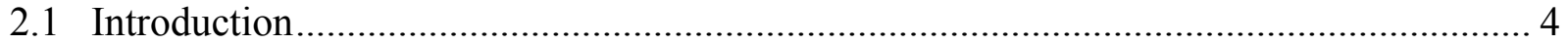

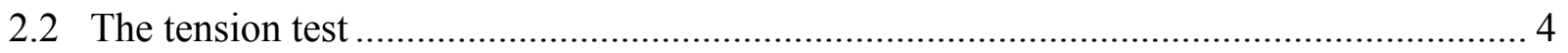

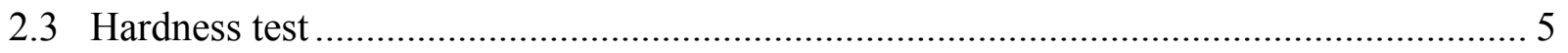

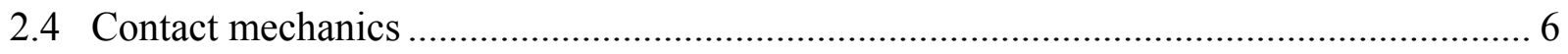

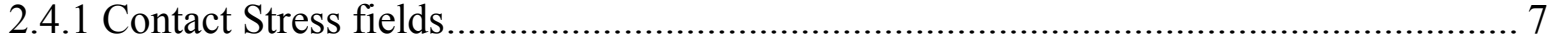

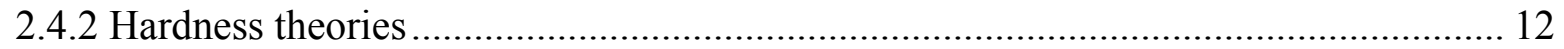

2.5 Instrumented Indentation Test ........................................................................ 22

2.6 Indentation Stress-Strain Response..................................................................... 27

2.7 Experimental method to obtain the indentation stress-strain curve............................... 29

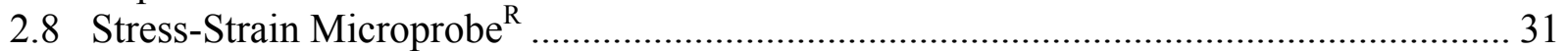

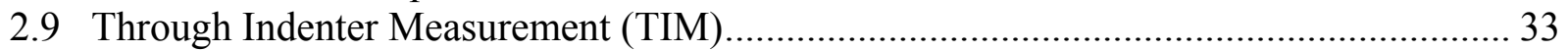

Chapter 3 SIMPLIFIED TIM INSTRUMENT ................................................................... 39

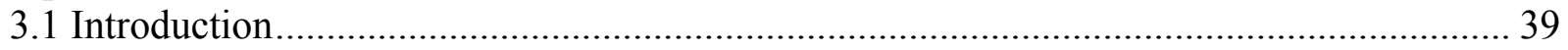

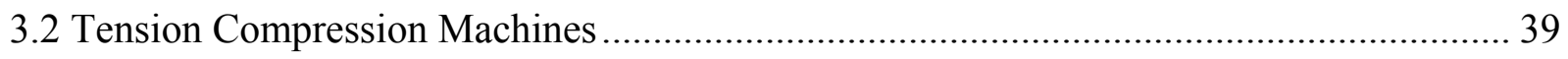

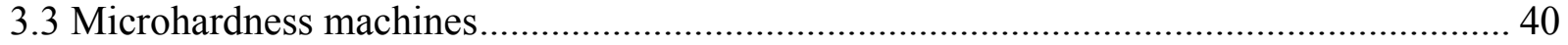

3.4 Instrumented Indentation Test ......................................................................... 42

3.5 Development of a simplified TIM Instrument. .................................................. 43

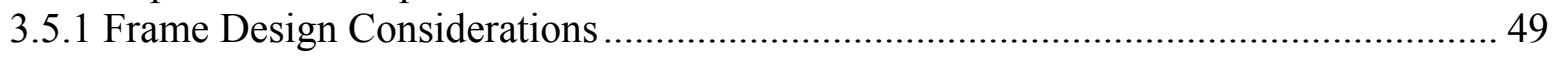

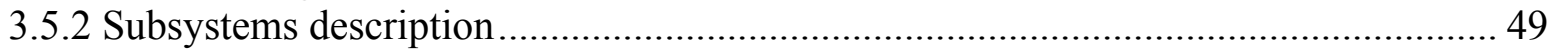

3.5.2.1 Mechanism to align the indenter normal to the specimen surface...................... 50

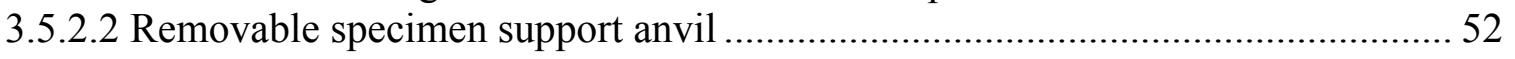

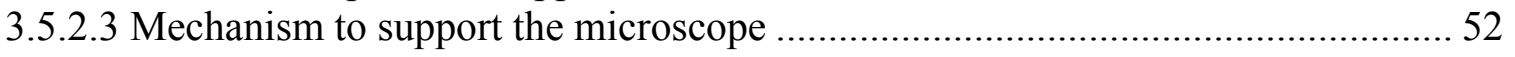

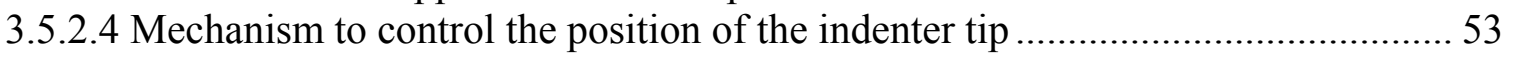

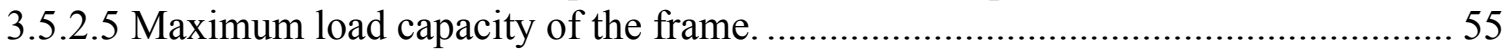

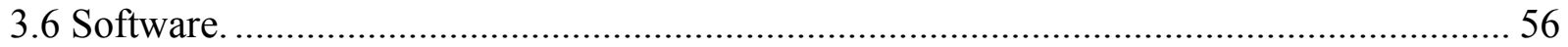

3.7 The Simplified Transparent Indenter Measurement Instrument Prototype. .................... 59

Chapter 4 RESULTS .....................................................................................................6. 61

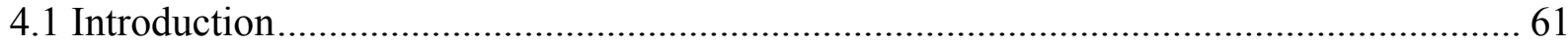

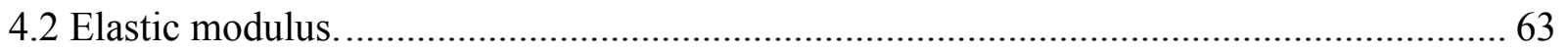

4.2.1 Elastic modulus determination based on the Newton's rings ............................... 63 


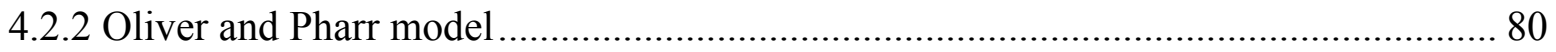

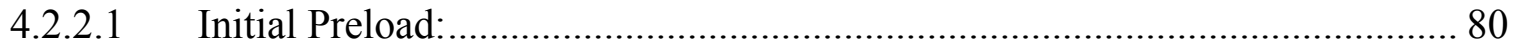

4.2.2.2 Single Cycle of Loading and Unloading.................................................. 81

4.2.2.3 Example of Initial Penetration Correction .................................................. 81

4.2.2.4 Correction by instrument compliance ................................................... 82

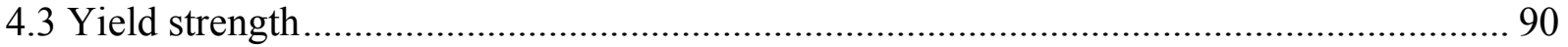

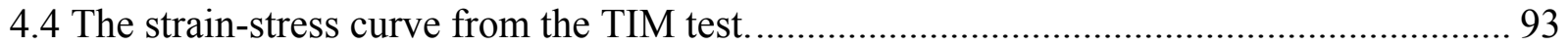

Chapter 5 SUMMARY AND FUTURE WORK................................................................. 95

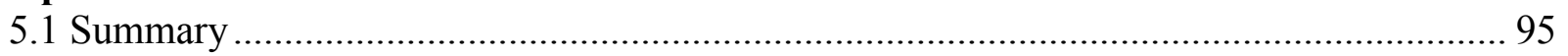

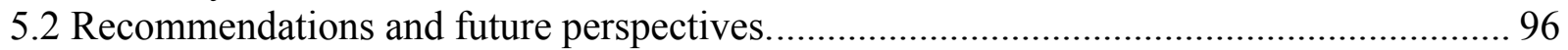

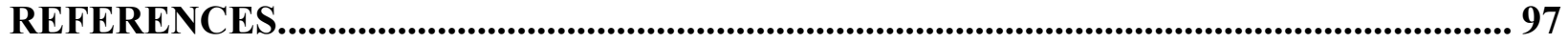

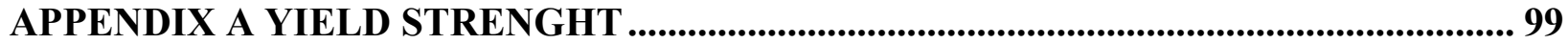

APPENDIX B APPLICATION TO BRITTLE MATERIALS ........................................... 102 


\section{LIST OF FIGURES}

Figure 2.1 Schematic of the interaction between a rigid spherical indenter and a specimen ......... 8 Figure 2.2 Schematic representation of the expanding cavity model. Figure adapted from

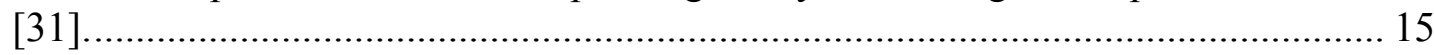

Figure 2.3 Example of the Meyer's exponent calculation using the experimental data provided by the TIM equipment.

Figure 2.4 Load versus displacement curve (Also called Compliance curve). Experimental data from TIM instrument. Material Aluminum 7075-T6. Indenter sapphire 1.5 mm diameter.

Figure 2.5 True Stress- True-Strain curve for Aluminum 6061-T6 compared to experimental data from depth-sensing indentation method. From [18].

Figure 2.6 Indentation stress-strain results from experimental and Finite Element method. Tungsten Carbide balls of different sizes have been used to obtain the experimental data [7]. 30

Figure 2.7 True-stress-True-Strain curve obtained from the ABI method, patented equipment "Stress-Strain Microprobe" [19] .......................................................................... 33

Figure 2. 8 Schematic optical TIM system from Dr. Kang's research group. Figure from

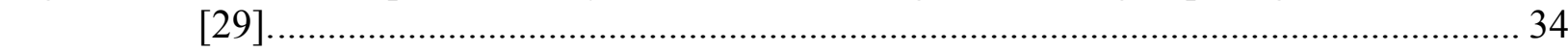

Figure 2.9 TIM testing procedure proposed by Dr. Kang's research group. Graph from [29]..... 35

Figure 2.10 Experimental arrangement to generate the Newton's Rings. Adapted from [30] ..... 37

Figure 2.11 TIM experimental set-up, showing the sapphire transparent half ball tip indenter... 38

Figure 2.12 Schematic to identify the Newton's Ring radius $r_{n}$, and the thickness of the air

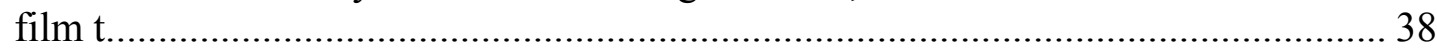

Figure 3.1 The simplified TIM instrument (a) scaled drawing (b) schematic view. .................... 45

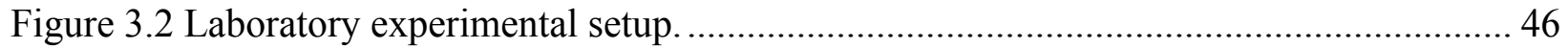

Figure 3.3 Electric, electronic and optical equipment components for the TIM......................... 47

Figure 3.4 Closed-up of the fundamental part of the TIM system............................................... 48

Figure 3. 5 Mechanism to position the indenter normal to the specimen.................................... 50

Figure 3. 6 Control mechanism to adjust the rotation of the indenter. The objective is to position the indenter normal to the specimen surface. ............................................ 51

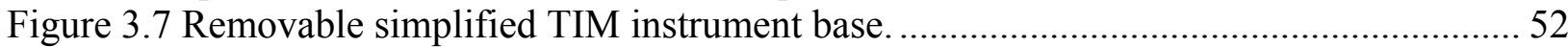

Figure 3. 8 Mechanisms to support the microscope. …………................................................ 53

Figure 3.9 Mechanism to control the position of the indenter...................................................5 54

Figure 3.10 A ProEngineer model of the proposed portable TIM equipment............................55 55

Figure 3.11 Trapezoid wave shape, showing the control parameters .......................................... 57

(Window provided by "Physik Instrumente"). ......................................................... 57

Figure 3.12 Control panel for the piezoelectric actuator, load cell and image acquisition............ 58

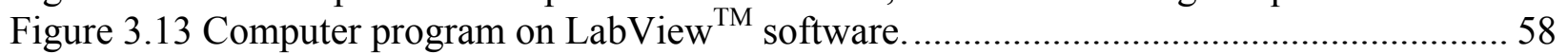

Figure 3.14 The Simplified Transparent Indenter Measurement Prototype............................... 59

Figure 3.15 The simplified TIM instrument prototype and accompanying electronic

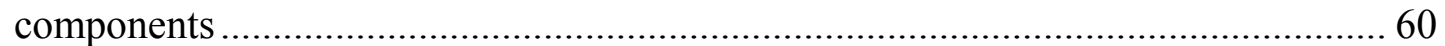

Figure 4.1 Raw data from the TIM instrument. Aluminum 7075-T6 ........................................ 62

Figure 4.2 The TIM instrument provides a) The load-piezoelectric tip displacement, and b) the image of the specimen surface with the radius of the Newton's rings. ............... 64

Figure 4.3 Coordinate system and the non-deformable spherical indenter, the specimen surface under no load is the same as the $\mathrm{x}$-axis. 
Figure 4. 4 Spherical indenter, specimen surface and Newton ring radius. 66

Figure 4. 5 Geometrical relations to determine the specimen surface position from the Newton's rings radius.

Figure 4.6 Simplified TIM's image and the associated graph after the present procedure were applied. 68

Figure 4.7 Aluminum alloy 7075-T6. Graphs (a) to (f) show the position of the specimen surface obtained by the Newton's ring method under different loads. The Hertz surface is superimposed to compare with the experimental data.

Figure 4.8 Contact radius obtained by the Newton's rings method in agreement with the Hertz equation for Aluminum alloy 7075-T6 75

Figure 4. 9 Experimental elastic modulus for Aluminum 7075-T6 obtained by the Newton's ring method...... 75

Figure 4.10 Inconel 783 Graphs (a) to (e) show the position of the specimen surface obtained by the Newton's ring method under different loads. The Hertz surface is superimposed for comparison with the experimental data. 76

Figure 4.11 Contact radius obtained by the Newton's rings method in agreement with the Hertz equation for Inconel 783.

Figure 4.12 Experimental elastic modulus for Inconel 783 obtained by the Newton's rings to determine the specimen surface position. 77

Figure 4. 13 Molybdenum Alloy 695. Graphs (a) to (e) show the position of the specimen surface obtained by the Newton's ring method under different loads. The Hertz surface is superimposed to compare with the experimental method.

Figure 4.14 Contact radius obtained by the Newton's ring method in agreement with the Hertz equation for Molybdenum alloy 695. 79

Figure 4.15 Experimental elastic modulus for Molybdenum Alloy 695 obtained by the Newton'srings method.

Figure 4. 16 Example of raw data from the simplified TIM instrument. 81

Figure 4.17 An example of the calculation of the initial penetration correction due to the initial contact preload of $1.2 \mathrm{~N}$. 82

Figure 4.18 Example of the unloaded part of the graph given as PZT displacement versus load. The raw data is corrected by initial penetration. 85

Figure 4.19 The inverse of contact stiffness is plotted against the inverse of the contact radius. 86

Figure 4.20 Depth-load curve for Aluminum 7075-T6. The raw data was corrected by initial penetration and system compliance.

Figure 4.21 A third of the unload curve corrected by the system compliance. ...................... 87

Figure 4.22 Example of elastic modulus by the Depth Sensing Indentation Method................. 89

Figure 4.23 The TIM experimental elastic modulus compared with the published value........... 89

Figure 4.24 Plot of force against residual impression radius used to calculate the Meyer's exponent. Meyer's Law F $=\mathrm{kd}^{\mathrm{m}}$ Material Aluminum alloy 7075-T6

Figure 4.25 Experimental indentation stress - strain for fully plastic region. Material Aluminum 7075-T6. TIM instrument with a sapphire ball indenter of $1.5 \mathrm{~mm}$ diameter.

Figure 4. 26 Plot of force against residual impression radius used to calculate the Meyer's exponent. Meyer's Law F $=\mathrm{kd}^{\mathrm{m}}$ Material Inconel Alloy 783 
Figure 4.27 Experimental indentation stress - strain. Material Inconel Alloy 783. TIM

instrument with a sapphire ball indenter of $1.5 \mathrm{~mm}$ diameter.

Figure 4.28 Experimental TIM instrument true-stress true strain compared with literature uniaxial true strain true stress curve. Material Aluminum 7075-T6........................ 93

Figure 4.29 Experimental TIM instrument true-stress true strain curve for Inconel Alloy 783 ... 94

Figure A. 1 Loading Slope / Unloading slope against force. Aluminum 7075-T6. 99

Figure A. 2 Loading slope portion of the load-piezoelectric displacement curve against force. Aluminum 7075-T6. 100

Figure A. 3 Loading slope portion of the load-piezoelectric displacement curve against load.

The load is shown until 2.0 N. Aluminum 7075-T6

Figure B. 1 Simplified TIM results for Molybdenum alloy 695. 102

Figure B. 2 Load-piezoelectric displacement curve unloading slope/loading slope versus Force for Molybdenum Alloy 695. 103

Figure B. 3 Loading slope of the load-piezoelectric displacement curve against force. Molybdenum Alloy 695. 103

Figure B. 4 Loading slope portion of the load-piezoelectric displacement curve. The load is shown until 2.0 N. Aluminum 7075-T6.

Figure B. 5 Simplified TIM instrument pictures for Molybdenum Alloy 695. 105 


\section{LIST OF TABLES}

Table 3.1 Example of Hardness methods existent for industrial use, adapted from [25]........... 41

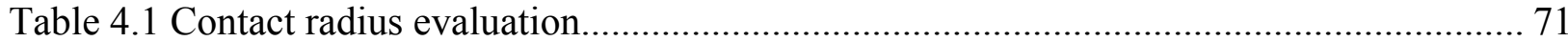

Table 4.2 Mechanical properties of the tested materials..................................................... 72

Table 4.3 Mechanical Properties by the optical method-Newton rings.................................... 94 


\section{NOMENCLATURE}

\section{Roman Symbols}

\begin{tabular}{|c|c|}
\hline a & Contact radius \\
\hline $\mathrm{E}$ & Elastic modulus of the material \\
\hline$E^{\prime}$ & Elastic modulus of the specimen \\
\hline $\mathrm{E}^{*}$ & Reduced Modulus \\
\hline $\mathrm{P}$ & Applied load. \\
\hline $\mathrm{R}$ & Combined radius \\
\hline $\mathrm{R}_{1}$ & Radius of body 1 in contact \\
\hline $\mathrm{R}_{2}$ & Radius of body 2 in contact \\
\hline $\mathrm{p}_{\mathrm{m}}$ & Mean pressure \\
\hline $\mathrm{r}$ & Distance from the center of the indentation \\
\hline $\mathrm{u}_{\mathrm{z}}$ & Displacement $\mathrm{z}$ direction \\
\hline $\mathrm{d}$ & Diameter of the residual impression on the surface of the specimen \\
\hline $\mathrm{H}$ & Hardness \\
\hline $\mathrm{C}$ & Constraint factor \\
\hline $\mathrm{Y}$ & Material's Yield strength \\
\hline $\mathrm{p}$ & Pressure within the core \\
\hline $\ln$ & Natural log \\
\hline A & Projected contact area \\
\hline $\mathrm{S}$ & Contact stiffness \\
\hline $\mathrm{B}$ & Empirically determined fitting parameter \\
\hline $\mathrm{m}$ & Empirically determined fitting parameter \\
\hline $\mathrm{h}_{\mathrm{f}}$ & Final displacement after complete the cycle \\
\hline $\mathrm{h}_{\max }$ & Maximum displacement \\
\hline $\mathrm{D}$ & Indenter diameter \\
\hline$d_{p}$ & Residual impression diameter on the $\mathrm{ABI}$ test \\
\hline $\mathrm{k}^{*}$ & Strength coefficient \\
\hline k' & Stiffness \\
\hline $\mathrm{k}_{\mathrm{t}}$ & Total stiffness \\
\hline $\mathrm{k}_{\mathrm{s}}$ & Sample stiffness \\
\hline $\mathrm{k}_{\mathrm{f}}$ & Frame stiffness \\
\hline $\mathrm{C}_{\mathrm{t}}$ & Total compliance \\
\hline $\mathrm{C}_{\mathrm{s}}$ & Sample compliance \\
\hline $\mathrm{C}_{\mathrm{f}}$ & Frame compliance \\
\hline $\mathrm{h}_{\mathrm{t}}$ & Total deformation \\
\hline $\mathrm{h}_{\mathrm{f}}$ & Frame deformation \\
\hline $\mathrm{h}_{\mathrm{s}}$ & Specimen deformation \\
\hline $\mathrm{h}_{\mathrm{c}}$ & Contact depth \\
\hline $\mathrm{h}$ & Total penetration. \\
\hline $\mathrm{n}$ & Strain hardening exponent. \\
\hline $\mathrm{n}^{*}$ & Material constant \\
\hline e & Material constant \\
\hline
\end{tabular}


y Material constant

\section{Greek Symbols}

$v \quad$ Poisson's ratio of materials

$v$ ' Poisson's ratio of indenter

$\delta \quad$ Material's parameter

$\varepsilon \quad$ Strain

$\varepsilon_{\mathrm{t}} \quad$ True strain

$\varepsilon_{\mathrm{p}} \quad$ Plastic Strain

$\sigma \quad$ Stress

$\sigma_{\mathrm{t}} \quad$ True Stress

$\sigma_{\mathrm{z}} \quad$ Stress on de $\mathrm{z}$ direction

$\beta \quad$ Constant, which depend on the geometry of the indenter

$\rho \quad$ Distance to the indenter center

$\lambda \quad$ Wavelength 


\begin{tabular}{ll} 
& \multicolumn{1}{c}{ Abbreviations } \\
ASTM American Society of Testing of Materials \\
ISO & International Standardization Organization \\
TIM & Transparent Indenter Measurement \\
IIT & Instrumented Indentation Test \\
WC & Tungsten Carbide \\
P/M & Powder Metallurgy \\
ABI & Automatic Ball Indentation \\
SSM & Stress Strain Microprobe \\
LVDT & Linear Variable Differential Transformer \\
DIN & Deutsches Institut für Normung E.V. \\
JIS & Japanese Standards Association \\
BK7 & Optical Glass \\
LCVR Liquid Crystal Variable Retarded \\
WVU & West Virginia University \\
mN & Millinewton \\
kN & Kilonewtons \\
gf & Grams force \\
N & Newton \\
Lbf & Pounds force \\
UTM & Universal testing machine \\
&
\end{tabular}




\section{Chapter 1 INTRODUCTION AND RESEARCH OBJECTIVE}

New applications and the development of materials at the nanoscale level, have motivated researchers to develop alternative material testing methods to the tension test, in order to obtain relevant mechanical properties at the nano or micro-scale level.

As an example of this, the Instrumented Indentation Test (IIT) was developed for the measurement of mechanical properties at small scale. The method was originated by Tabor in the 1950's [12], and it consists of continuous control, monitoring and recording of loads and displacements of material under indentation. On the IIT method, the contact area is determined indirectly by the area function of a well-defined indenter tip shape, as the indenter penetrates the sample surface. During the past decade, the development of the IIT instrument has been greatly improved due to the advancement in software and electronics used in control, sensitivity and data acquisition.

The IIT equipment is sensitive to environmental effects and must be isolated from external sources of vibration and airflow, like the ones from air conditioning sources or from opening and closing doors. These environment constraints of the nanoindenter make it impossible to have a portable instrument.

One major drawback of IIT method is the requirement of accurate depth sensing measurement, which must exclude the effect of system compliance and have the measurement resolution in the nanometer scale. On the other hand, one of the purposes of depth sensing measurement is to determine the indented area. The idea of using a transparent indenter to measure directly the indented area was first conceived by Dr. Kang and subsequently a Transparent Indenter Measurement (TIM) method was developed [29, 37]. 
In this research, a simplified version of the original TIM method was designed and built. White light is used to illuminate the sample surface, through a sapphire spherical indenter tip. The contact area is measured as the piezoelectric actuator moves the optical transparent indenter against the sample surface. The indenter elastically contacts or plastically deforms the material. The experimental data includes the load-piezoelectric tip displacement graph and the indented area.

This dissertation is organized in the following way:

Chapter two includes a review of standards for tension and hardness tests, as described in Sections 2.2 and 2.3. The standards are a source of information and requirements to facilitate the design of the simplified TIM instrument. A brief theoretical background of contact mechanics is presented in Section 2.4. This section describes the application of contact mechanics to indentation, starting with the Hertz theory for elastic contact of solids and followed by the concept of the expanding cavity model for the elastic-plastic region is presented. Special attention is devoted to the depth sensing indentation method, and the theory behind it. The models used in the development of the procedures to obtain the Young's modulus on this research are also presented.

Section 2.5 discusses the current experimental methods to obtain the indentation stressstrain curve. Chapter two includes a review of the fundamental equations and capacities of the commercial equipment, in particular the registered trademark "Stress-Strain Microprobe" (Section 2.8). The method used under this trademark calculates the true stress-true strain is called "Automatic Ball Indentation" [19]. This patented equipment is used to obtain the true stress-true strain curve of a metal using depth sensing indentation concepts, at the macroscopic level. 
Chapter Three is completely dedicated to describe the details of the design of the equipment proposed in this research. This chapter starts with a review of the characteristics of existing tension, microhardness and depth sensing indentation equipment (Sections 3.2 and 3.3). This is relevant information that has been used as a guide in the design process of the frame for the simplified TIM equipment. Descriptions of the frame that supports the piezoelectric actuator, the optical transparent indenter, the microscope and camera, as well as specifications and characteristics of sub mechanisms and their functions, are described in Section 3.5. The frame was designed using ProEngineering ${ }^{\mathrm{TM}}$ (Computer Aided Design software) and is composed of sub-assemblies, which must provide high precision position control of the indenter as well as accurate alignment of the indenter with respect to the test specimen. Section 3.5.2 describes the mechanism that allows the use of different indenters and load cells. This mechanism provides the capability to conduct indentation test on both the elastic and plastic range. The design of the microscope support assures that the microscope objective lens will always be in focus during the indentation test.

Chapter Four describes the test procedure used in this research to obtain the stress-strain curve. A new procedure was developed for the elastic modulus determination, and a second procedure involves the use of concepts of depth sensing indentation.

Finally, some conclusions derived from the results obtained in this work and proposed future works using the simplified TIM instrument, are presented in Chapter Five. 


\section{Chapter 2 LITERATURE REVIEW}

\subsection{Introduction}

For mechanical property measurements, it is desirable to develop new methods, or to modify the existing testing methods to obtain mechanical properties of small-size material samples or thin-film coatings [9].

The traditional methods of tension and hardness tests are reviewed from the point of view

of the testing standards. The testing standards can provide an understanding of specifications and requirement for the development of new material testing instruments. Traditional experimental methods to obtain the indentation stress-strain curve are also discussed. The indentation testing instruments based on the depth sensing indentation concept are presented.

\subsection{The tension test}

The most common test used to find material properties is the "Tension Test" in which a specimen is loaded to failure and material properties such as elastic modulus, yield strength, ultimate tensile strength, modulus of resilience, and toughness are obtained.

The tension test has been standardized $[1,5,6]$ to cover aspects such as testing apparatus, loading rate, gripping devices, displacement measuring devices, and the like. The geometric and other properties of the specimens are also specified by the standard, some of which include material conditions for different sample or specimen shapes like plate-type, sheet-type, round specimen, specimens for sheet, strip, flat wire and plate or rectangular bar [1].

The standard provides specific requirements for the determination of the mechanical properties. For instance, speed of testing for yield strength is normalized. There are normalized methods to determine the yield strength such as the offset method or the extension-under-load 
method. Additionally, there are standard procedures to calculate the yield point such as halt-ofthe-load, autographic diagram, strain rate, and extension-under-load-method.

\subsection{Hardness test}

In the hardness test an indenter is pressed into the surface of the material under a specific load for a definite amount of time. A measurement of the size or depth of the indentation is made [2]. The Hardness value, depend on the applied load in a specific manner for a specified penetration shape and a determined amount of time.

The work of this research is based on the following statement: the hardness test and the tensile test measure the resistance of a metal to plastic flow. Therefore, the results of these tests may be correlated to each other [2].

The hardness tests are standardized. For example, standard test methods for Brinell hardness of metallic materials have the designation ASTM E-10-xx [3]. The ASTM Standard, “Test Method for Microhardness of Materials", ASTM E 384_xx [4], can be used as a guide for the development of the TIM frame support and the procedure to test the samples. The force range for the microhardnes test is from 1 to 1000 grams of force. The scales for the measurement are known as Knoop and Vickers hardness number.

The standard defines aspects of the indentation test such as apparatus and testing machine, load application, and specific details for the test procedure. For example the indenter must contact the specimen, at a velocity between $15-70 \mu \mathrm{m} / \mathrm{s}$ while the time of applied load is to between 10 to 15 seconds [4]. This normalized time will be used as a holding time during the tests on this research. The holding time is the time that the indenter is in contact with the sample at maximum load. 
For the microhardness test, the external conditions are important. For instance, the external vibrations must be controlled, or the sources of environmental vibration must be avoided.

The Standard offers information about indenter characteristics, microscope and ocular objectives, and other specific conditions. For a residual indentation length less than $76 \mu \mathrm{m}$ the magnification minimum value must be 400. Aspects of illumination are also covered.

\subsection{Contact mechanics}

The indentation test is an application of the theory of the contact between solids, that is, the act of bringing two bodies in contact. Two terms are important to this process. The term "indenter" is the body to which the loading force is applied, rigid and non-deformable by the interaction with the second body, which is the "specimen" or the material, whose mechanical properties are to be determined. The mechanical interaction between the indenter and the specimen will be purely elastic, elastic-plastic, plastic or irreversible as a function of the applied load or indenter penetration. To understand and to interpret what is measured during the indentation process, a review of the theories that describe the interaction between forces and material behavior is presented.

The history of contact mechanics starts with studies conducted by Hertz [7]. The fields of electricity and optics were related to the origin of "contact mechanics". In 1880, Heinrich Hertz became Helmontz's assistant. He was interested in the phenomenon of Newton's rings, the possible influence of elastic distortion of contact lenses and the distribution of pressure between the two contacting surfaces [31]. Hertz published his work under the title "On the contact of elastic bodies." Due the importance of this work, the Hertz's boundary conditions are listed below [7]: 
a. "The displacements and stresses must satisfy the differential equations of equilibrium for elastic bodies, and the stress must vanish at a great distance from the contact surface - that is the stresses are localized."

b. "The bodies are in frictionless contact."

c. "At the surface of the bodies, the normal pressure is zero outside and equal and opposite inside the circle of contact."

d. "The distance between the surfaces of the two bodies is zero inside and greater than zero outside the circle of contact."

e. "The integral of the pressure distribution within the circle of contact with respect to the area of the circle of contact gives the force acting between the two bodies."

Conditions "a" to "e" play an important role in understanding the relations between the theoretical and experimental data. The full contact stress field appears to have been first calculated by Huber in 1904 and Fuchs in 1913 [7]. In brittle solids, the stresses responsible for the conical cracks are the radial tensile stresses on the specimen surface. The cracks are called "Hertzian cone cracks" [8].

\subsubsection{Contact Stress fields}

Figure 2.1 shows a cross-section of a frictionless contact of a sphere with radius $\mathrm{R}$ at a normal load $P$ on a flat continuum specimen. The radius of contact is $a, h_{\mathrm{t}}$ is the total depth of penetration, or total displacement of the surface, $h_{e}$ is the distance from the free surface to the radius of contact, and $h_{p}$ is the distance from the radius of contact to the total depth. 


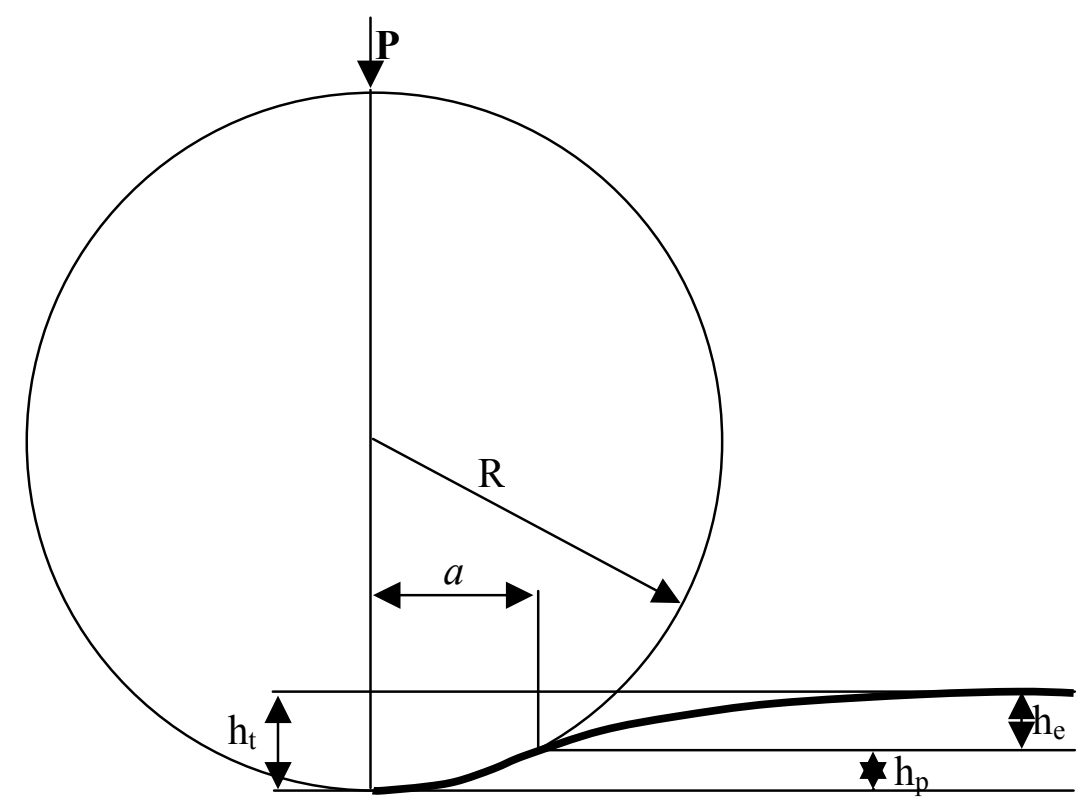

Figure 2.1 Schematic of the interaction between a rigid spherical indenter and a specimen

The Hertz's elastic equations for a sphere of radius $\mathrm{R}$ at a normal load $P$ are well documented [7, 8]. Hertz demonstrated that the contact radius, $a$, is given by Equation (2.1).

$a^{3}=\frac{4 k P R}{3 E}$

where

$E=$ Young's Modulus of the specimen

$P=$ Applied load

$R=$ Combined radius

$\mathrm{k}=\left(\frac{9}{16}\right)\left[\left(1-v^{2}\right)+\left(1-v^{\prime 2}\right) \frac{\mathrm{E}}{\mathrm{E}^{\prime}}\right]$

where

$v=$ Poisson's ratio of the specimen 
$v^{\prime}=$ Poisson's ratio of the indenter

$E^{\prime}=$ Young's modulus of the indenter

The combined radius is determined by the following expression:

$\frac{1}{R}=\frac{1}{R_{1}}+\frac{1}{R_{2}}$

where

$R_{1}=$ radius of the body 1

$R_{2}=$ radius of the body 2

For a rigid indenter, the sample is considered to be a flat surface. From Equation (2.3), $R_{2}$ goes to infinity and the combined radius is equal to the indenter radius.

The concept of reduced modulus was introduced by Tabor [12]. The reduced modulus is a composite value used to account for the non-rigid tip interaction, or the elastic interaction between the tip and the specimen surface. The two interacting materials are assumed to behave as two springs connected in series, and the reduced modulus is expressed as [9]:

$\frac{1}{E^{*}}=\frac{\left(1-v^{2}\right)}{E}+\frac{\left(1-v^{\prime 2}\right)}{E^{\prime}}$

where

$E^{*}=$ Reduced Modulus

Hertz was concerned with the nature of the localized deformation and the distribution of pressures between two elastic solids. By analogy with the theory of electric potential, Hertz deduced that an ellipsoidal distribution of pressure would satisfy the boundary conditions of the problem and found that, for the case of the sphere, the required distribution of pressure is given by the following equation [7]: 
$\frac{\sigma_{z}}{p_{m}}=-\frac{3}{2}\left[1-\frac{r^{2}}{a^{2}}\right]^{1 / 2} \quad r \leq a$

where

$\sigma_{\mathrm{z}}=$ Stress on de $\mathrm{z}$ direction

$\mathrm{p}_{\mathrm{m}}=$ Mean pressure

$a=$ Contact radius

$\mathrm{r}=$ Distance from the center of indentation.

The displacement of points on the surface of the specimen within the contact circle measured with respect to the original specimen's free surface is given by [7]:

$$
u_{z}=\frac{1-v^{2}}{E} \frac{3}{2} p_{m} \frac{\pi}{4 a}\left(2 a^{2}-r^{2}\right) \quad r \leq a
$$

Outside the contact circle the displacement of surface points is given by [7]:

$$
u_{z}=\frac{1-v^{2}}{E} \frac{3}{2} p_{m} \frac{1}{2 a}\left[\left(2 a^{2}-r^{2}\right) \sin ^{-1} \frac{a}{r}+r^{2} \frac{a}{r}\left(1-\frac{a^{2}}{r^{2}}\right)^{1 / 2}\right] r \geq a
$$

For the special case of a rigid indenter, Equation (2.6), at $r=0$ gives the penetration depth beneath the original specimen surface and also the distance of mutual interaction between two points in both the indenter and specimen. An important observation for the calculation of the elastic modulus is that Equation (2.6), the amplitude of the depth $[\mathrm{r}=a]$, is exactly one half of the maximum depth [7].

During the contact time, the contact surface is a function of the two original surfaces and the elastic properties of the two contacting materials. For the case where the indenter and the 
specimen are not rigid, Fisher-Cripps [7] shows that the deformation must account the influence of the non-rigid indenter deformation. Equations (2.6) and (2.7) are modified to:

$$
\begin{aligned}
& u_{z}=\left(\frac{1-v^{\prime 2}}{E^{\prime}}+\frac{1-v^{2}}{E}\right) \frac{3}{2} p_{m} \frac{\pi}{4 a}\left(2 a^{2}-r^{2}\right) \quad r \leq a \\
& u_{z}=\left(\frac{1-v^{\prime 2}}{E^{\prime}}+\frac{1-v^{2}}{E}\right) \frac{3}{2} p_{m} \frac{1}{2 a}\left[\left(2 a^{2}-r^{2}\right) \sin ^{-1} \frac{a}{r}+r^{2} \frac{a}{r}\left(1-\frac{a^{2}}{r^{2}}\right)^{1 / 2}\right] r \geq a
\end{aligned}
$$

The mean contact pressure, a quantity of considerable physical significance is defined as:

$$
\mathrm{p}_{\mathrm{m}}=\frac{4 \mathrm{P}}{\pi \mathrm{d}^{2}}
$$

The mean contact pressure in many books is defined as the Meyer hardness and it is defined as the force divided by the projected area of contact [7].

If Equations (2.1) and (2.2) are combined, the elastic modulus of the material is a function of the contact radius and the material properties of the indenter, then:

$$
E=\frac{1-v^{2}}{\left[\frac{4}{3 R} \frac{a^{3}}{P}-\frac{\left(1-v^{\prime 2}\right)}{E^{\prime}}\right]}
$$

If Equations (2.9) and (2.11) are combined in order to obtain de surface deformation as a function of the contact radius $a$, Equation (2.12) is obtained. Equation (2.12) is only a function of the contact area and is not a function of any material parameter. This equation will be used in the proposed method to find the elastic modulus by the use of the Newton's rings. Equation (2.12) is independent of the elastic parameters of the material under test.

$$
u_{z}=\frac{1}{\pi R}\left[\left(2 a^{2}-r^{2}\right) \sin ^{-1} \frac{a}{r}+r^{2} \frac{a}{r}\left(1-\frac{a^{2}}{r^{2}}\right)^{1 / 2}\right] r \geq a
$$




\subsubsection{Hardness theories}

To continue with the present discussion the concept of constraint factor, related to the concept of hardness, will be introduced. The most famous criterion of hardness was proposed by Hertz [7] who postulated that... "An absolute value of hardness is the least value of pressure beneath the spherical indenter necessary to produce a permanent set at the center of the area of contact". The magnitude of the mean contact pressure $p_{m}$ at which there is no increase on the contact pressure with increasing indenter load is related to the hardness number $H$ of the material. This definition is valid for techniques that utilize the projected contact area. However, experiments show that the mean pressure between the indenter and the specimen is directly proportional to the material's yield, or flow stress in compression and it can be expressed as [7]:

$$
\begin{aligned}
& \mathrm{H} \approx \mathrm{CY} \\
& \text { where } \\
& \mathrm{C}=\text { constraint factor } \\
& \mathrm{Y}=\text { Flow stress of the material }
\end{aligned}
$$

The concept of constraint factor is related to the definition of hardness. The two components of hardness are: (a) a constraint factor for the test and (b) the uniaxial flow stress of the material being tested [10]. Considerable scientific work has been done on the constraint factor, in order to explain its cause and value based on the indenter geometry and mechanical properties of the test specimen [7]. The constraint factor concept was used by Prandtl in 1920 [10], who compared the blunt hardness indenter to the mean stress on a two dimensional punch required for the onset of plastic flow beneath the punch. Hill generalized the concept in 1947 $[10]$. 
In an indentation process, the indentation stress field is bound by the surroundings materials, and there is a considerable hydrostatic component. The mean contact pressure in an indentation test is then higher than the required to initiate yield in a uniaxial compressive test, this is so, since it is the shear component of stress the responsible for plastic flow [7].

For ductile materials, the hardness is 2.8 to 3.2 times their uniaxial flow stress values. The increase is primarily due to the fact that the plastic zone surrounding the indenter is confined within a large volume of material that can be either elastic or rigid [10].

Tabor [12] compares the stress-strain characteristics of metals with the hardness curves, and introduces the concept of "indentation stress" and "indentation strain" when the mean contact pressure, $\mathrm{p}_{\mathrm{m}}$, what can be escalated to indentation stress, is plotted versus the ratio between the residual impression over the indenter radius $(\mathrm{a} / \mathrm{R})$. The curve obtained from the indentation strain versus the indentation stress is similar to the stress-strain curve in the uniaxial tension test.

The following is a discussion of the concepts of indentation stress-strain curve, constraint factor and the uniaxial compressive yield stress $(\mathrm{Y})$ of the material. The indentation response of the material can be divided into three regions [7].
a. $\mathrm{p}_{\mathrm{m}}<1.1 \mathrm{Y}$
b. $1.1 \mathrm{Y}<\mathrm{p}_{\mathrm{m}}<\mathrm{CY}$
c. $\mathrm{p}_{\mathrm{m}}=\mathrm{CY}$.

In region (a), the response is fully elastic, it corresponds to the initial application of the load and the elastic response can be predicted by the Hertz equations [7].

$$
\mathrm{p}_{\mathrm{m}}=\left(\frac{3 \mathrm{E}}{4 \pi \mathrm{k}}\right) \frac{\mathrm{a}}{\mathrm{R}}
$$




$$
k=\frac{9}{16}\left[\left(1-v^{2}\right)+\left(1-v^{\prime 2}\right) \frac{E}{E^{\prime}}\right]
$$

In regime (a) no damage or permanent deformation is done on the surface, once the load is removed. For regions (b) and (c), Tabor [12] applied the Tresca or the Huber-Von Misses yield criterion and found that the maximum shear stress occurs at a depth of half of the center of the circle of contact $(0.5 a)$, with a principal shear stress approximately equal to $0.47 p_{m}$, where $p_{m}$ is the mean contact pressure. The plastic flow will occur when the shear stress equals $Y / 2$. That is when $0.47 p_{m}$ equal $0.5 Y$ so that the plastic deformation starts when [12]

$$
\mathrm{p}_{\mathrm{m}}=1.1 \mathrm{Y}
$$

For region (b), the onset of plastic deformation according to Tabor [12] begins when $p_{m}=$ $1.1 Y$, and occurs at a localized region beneath the surface constrained by the surrounding elastic material. The rest of the material is deformed elastically.

In region (c), the plastic zone increases the size as the indenter penetrates the material and the contact area grows in size. The plastic zone eventually reaches the material's surface. The contact area increases the size at a rate, that, an increase in indenter load produces no additional change in the mean contact pressure. This condition has been called a definition of hardness when the projected contact area is used as a method for hardness measurements [7].

The characteristics of the indenter and the assumed response of the material under test are the base for the theoretical approaches of hardness [7]. For blunt indenters, the specimen responds in an elastic-plastic manner, and plastic flow is usually described in terms of the elastic constraint offered by the surrounding material [7]. We discuss here only the expanding cavity model as a theoretical approach for the elastic-plastic region. 
As the indenter tip contacts the specimen surface, an elastic response at low loads is expected. When the indenter penetrates the surface, its volume must be accommodated and the type of response depends on the indenter tip type, whether it's blunted or sharp. In the expanding cavity model the volume under the indenter is represented by a hemispherical core subjected to an internal pressure, which is directly related to the mean contact pressure [7].

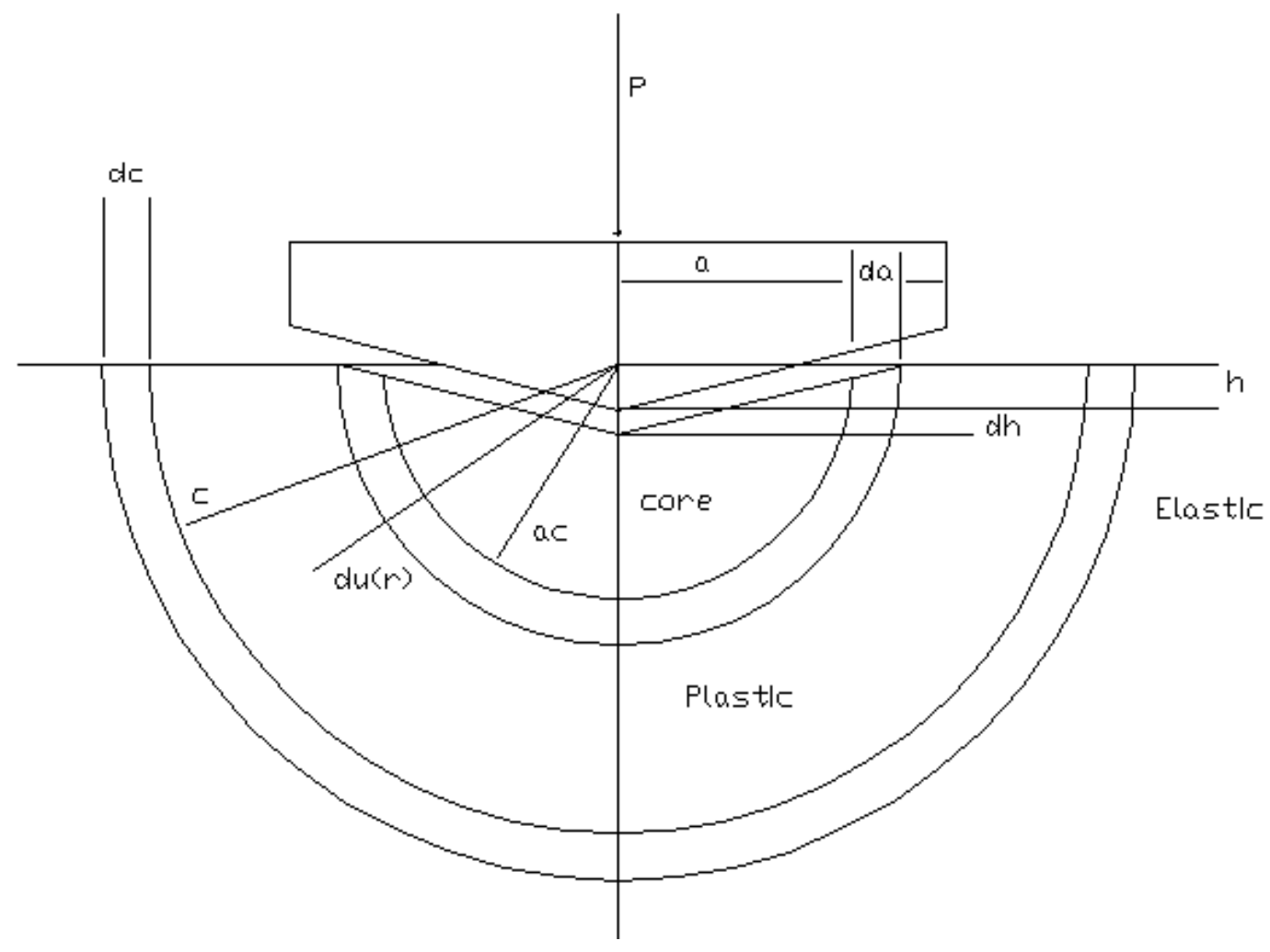

Figure 2.2 Schematic representation of the expanding cavity model. Figure adapted from [31].

As the tip is further indented into the surface by a quantity, $d h$, the contacting area between the indenter and the sample increases, producing an increment in the hydrostatic core of radius $a_{c}$. The core is confine by a plastic zone of radius (c) and that plastic zone is constrained by the surrounded material in an elastic state of deformation. The core grows, in size $\left(d_{a}\right)$ and is associated to the penetration of the indenter tip. The volume displaced by the indenter 
accommodated by radial movement of particles du(r) increases the size of the plastic zone at the core boundary [7].

The expanding cavity model is based on the experimental observation, where the deformation follows a hemispherical radial mode. The cavity is replaced by an incompressible hemispherical core of material located beneath the indenter [7]. The radius core $\left(a_{c}\right)$ is equal to the radius of the circle of contact between the indenter and the material. Encircling the core, there is a deformable plastic zone of radius $c$. As the indenter penetrates the material surface an amount, $d h$, the core radius expands da, and the plastic zone expands dc, as shown in the Figure 2.2. Using the concept of constant volume, as the indenter penetrates the material, there is a radial outward movement of particles located at the core boundary that causes the expansion of the plastic zone. For the case of spherical indenters, the elastic stress distribution within the specimen is directly proportional to a [7]:

$$
\frac{p}{Y}=\frac{2}{3}\left[1+\ln \left[\frac{\left(\frac{E}{Y}\right) \frac{1}{2} \frac{a}{R}+4(1-2 v)}{6\left(1-v^{2}\right)}\right]\right]
$$

Where

$p=$ Pressure within the core

$\ln =$ Natural $\log$.

Hertz attempted to use his theory to give a definition of hardness of a solid in terms of the contact pressure that initiates plastic yielding, but failed due to the difficulty of detecting the point of first yield under the action of contact stress [31]

From the onset of plastic deformation, the yield strength can be assessed [31]. Johnson [31] proposed that the load at which plastic yield begins is related to the yield point of the softer material in a simple tension or shear test, through an appropriate yield criterion. For axis- 
symmetric contact of solids of revolution, using the Tresca criterion, the value of $p_{o}$ for yield is given by [31]

$$
p_{0}=\frac{3}{2} p_{m}=3.2 k=1.60 Y
$$

where

$\mathrm{k}=$ Constant

using the von Mises criterion, the value of $p_{o}$ is:

$$
p_{o}=2.8 k=1.60 Y
$$

The load at which yield is initiated is related to the maximum contact pressure as:

$$
P_{y}=\frac{\pi^{2} R^{2}}{6 E^{*^{2}}}\left(p_{0}\right)_{y}^{3}
$$

where

$p_{o}=$ Maximum contact pressure

Using the Johnson critical threshold values for load and depth, Wolf [32] developed two interesting relations for the contact radius and the depth

$$
\begin{aligned}
& r_{c}=\left(\frac{\pi R}{2 E}\right)\left(\frac{Y}{0.31}\right)=5.07\left(\frac{Y}{E}\right) R \\
& \delta=\frac{r_{c}}{R}=26\left(\frac{Y}{E}\right)^{2} R
\end{aligned}
$$

where

$$
\delta=\text { Depth }
$$

Wolf [32] concludes that for spherical indentation, Equation (2.19) implies that the yield starts at a definite value, this is consistent with the Kick's theorem of proportional material 
resistance (similarity theorem) which states that: The material response to impressions with constant ratio i.e. contact radius/sphere radius, is always the same.

From the point of view of experimental work, Hertz's question of “exactly how can we estimate when the sample has yielded?" remains unanswered to this day. Herbert [18] explored three methods. These methods look for a deviation from the Hertz elastic behavior. All three methods were equally poor performers in terms of providing any conclusive evidence of the yield point [18].

The first is the relation between the total displacement in the sample and the contact depth. This is an application of Equation (2.6). The relation $h_{c} / h=0.5$ should be constant until the material yield [18], given by:
a.) $\frac{h_{c}}{h}=0.5$
where
$\mathrm{h}_{\mathrm{c}}=$ Contact depth
$\mathrm{h}=$ Total penetration.

The TIM method directly measures the area of indentation, not the depth of penetration. Errors will be introduced if this approach is used to calculate the yield strength of the material using the TIM method.

b.) For small displacements $\left(2 h_{c} R>>h_{c}{ }^{2}\right)$, this equation is used to determine when the yield has occurred [18]

$$
\mathrm{h}_{\mathrm{c}}=\frac{\mathrm{P}^{2 / 3}}{\left((8 \sqrt{2} / 3) \mathrm{E}_{\mathrm{r}}{ }_{\mathrm{r}} \mathrm{R}\right)^{2 / 3}}
$$

where 
$\mathrm{h}_{\mathrm{c}}=$ Contact depth

Again this method is based on penetration depth measurement and is not suitable for the TIM method.

c) Loading/unloading slope

The last method is easily implemented on the TIM instrument and will be investigated.

The finite element investigation by Mesarovic and Fleck [14], indicate that the first evidence of yielding occurs when $\mathrm{p}_{\mathrm{m}} / \sigma_{\mathrm{y}}=1.6$ in contrast to Tabor relation of $\mathrm{p}_{\mathrm{m}} / \sigma_{\mathrm{y}}=1.1$. The experimental results of [18] agree with Mesarovic's work for the determination of the yield strength of Aluminum 6061-T6.

A method proposed recently by Konda [21], which uses the magnitude of the force and the diameter of the residual impression to calculate the strain-hardening exponent by the use of the Meyer's equation. The material strain hardening value is substituted in the power law model of the strain-stress curve and this determines the yield strength of the material. This procedure was implemented for Aluminum 7075-T6 having good agreement.

The yield strength and the strain-hardening exponent were derived from experimental data by using the contact diameter and applied load during the loading and unloading cycles of indentation, through the following models:

Meyer's law

$$
\mathrm{P}=\mathrm{ed}^{\mathrm{n} *}
$$

where

$\mathrm{P}=$ Load

$\mathrm{d}=$ Contact diameter 
$\mathrm{n}^{*}, \mathrm{e}=$ Material's constants

Tabor's empirical equations:

$$
\begin{aligned}
& \varepsilon=0.2 \frac{d}{D} \\
& \sigma_{i}=\frac{p_{m}}{2.8} \\
& p_{m}=\frac{4 P}{\pi d^{2}}=\text { Meyer hardness } \\
& \mathrm{n}^{*}=\mathrm{n}+2
\end{aligned}
$$

where

$\varepsilon=$ True strain

$\sigma=$ True stress

$\mathrm{n}=$ Strain hardness exponent

The intersection of the linear elastic line with the power fit stress-strain curve gives the yield stress of the material.

$$
Y=\left(\frac{\alpha}{E^{n}}\right)^{\left(\frac{1}{1-n}\right)}
$$

where

$\alpha=$ Material constant

$\mathrm{n}=$ Strain hardening exponent.

The true stress - true strain curve can now be generated from the experimental data of the simplified TIM instrument. Figure 2.3 shows the application of Konda's approach [21]. The 
graph shows the calculation of the Meyer's coefficient by plotting the force versus the residual impression. A value of 2.0867 was obtained for the Meyer's exponent.

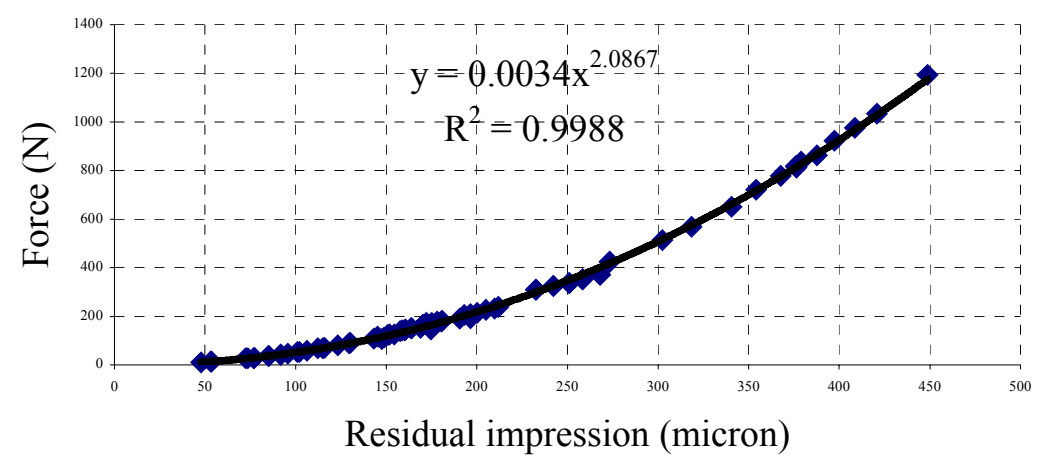

Figure 2.3 Example of the Meyer's exponent calculation using the experimental data provided by the TIM equipment.

In Konda's approach a variation of the previous method was implemented showing improvement in the results. The true stress correlated with the mean pressure underneath the indenter $p_{m}$ proposed by Tabor. Has been modified to the following form [17]

$\sigma_{t}=\frac{p_{m}}{\psi}$

where

$\psi=$ Constraint factor

The constraint factor $\psi$, is represented as a function to another parameter $\Phi[17]$

$$
\psi=\left\{\begin{array}{lr}
C_{1} & \Phi \leq 1 \\
C_{1}+C_{2} \ln \Phi & 1 \prec \Phi \leq C_{3} \\
\psi^{c} & \Phi \succ C_{3}
\end{array}\right\}
$$

Taljat [17] defines the constants as: 


$$
\mathrm{C}_{1}=1.11
$$$$
\mathrm{C}_{2}=0.536
$$

$\mathrm{C}_{3}=27.3$

$\Phi=\tan (\vartheta) \mathrm{E} / \sigma_{\mathrm{t}}=4 \mathrm{Eh}_{\mathrm{ps}} /\left(\mathrm{d}_{\mathrm{t}} \sigma_{\mathrm{t}}\right)$

$\vartheta=$ Angle between the tangent to the indenter at the indentation edge and the original surface.

$\mathrm{h}_{\mathrm{ps}}=$ depth

$d_{t}=$ Calculated diameter of the intersection between the indenter and the original material surface under loading.

$\sigma_{\mathrm{t}}=$ True stress

$\psi^{\mathrm{c}}=$ Constraint factor for the fully plastic region.

Reference [17] shows that the constraint factor for fully plastic region is dependent on the strain hardening exponent, $n$. For a strain hardening material this can be written as [17]:

$$
\psi^{c}=\frac{p_{m}}{\sigma_{t}}=\frac{6}{2+n}\left(\frac{40}{9 \pi}\right)^{n}
$$

Equation (4.25) will be used in Section 4.4 to calculate the constraint factor in order to obtain the true stress from the mean pressure provided by the TIM experimental data.

\subsection{Instrumented Indentation Test}

A relative new and powerful mechanical testing method employs high-resolution instrumentation to continuously control, monitor and record the loads and displacement during an indentation process. During the indentation cycle, the indenter loads and penetrates the material and unloads or withdrawn from the material. Depth Sensing Indentation is also known 
as instrumented indentation testing (IIT), continuous-recording indentation, ultra low load indentation and nanoindentation. Typical mechanical properties can be derived from the indentation load-displacement curve analysis obtained during the test.

Using the load-displacement curve, Oliver and Pharr [13] developed a method to calculate the hardness and the elastic modulus of the materials with an error of $10 \%$ or better [20]. Other methods have been developed to calculate the yield stress and strain hardening exponent of the materials $[18,19]$. The method of IIT is widely applied in many fields to find mechanical properties of ceramics, polymers, dental implantations, biological tissues and cells. Researchers use the depth sensing indentation method in many fields, and most material properties that can be measured in a uniaxial tension or compression test can also be conceivably measured, or estimated using the Depth sensing indentation method [20].

Topics related to the Depth Sensing Indentation such as testing equipment, types of tip indenters, aspects related to experimental practice and calibrations, are now standardized [22].

As seen in Figure 2.1, as the indenter is driven into the material, both elastic and plastic deformation processes occurs. The indenter penetration produced a deformation that is conformed to the shape of the indenter with a radius of circle of contact, a, and depth $\mathrm{h}_{\mathrm{c}}$. When the load is removed, only the elastic portion of the indentation displacement is recovered.

Figure 2.4 represent the displacement-load indentation process obtained from the TIM equipment for one cycle of indentation. The quantities are maximum load $\left(\mathrm{P}_{\max }\right)$. The maximum depth $\left(\mathrm{h}_{\max }\right)$, final or residual depth after unloading $\left(\mathrm{h}_{\mathrm{r}}\right)$, depth of residual impression for un equivalent punch $\left(h_{p}\right)$ and slope of the upper portion of the unloading curve $S=d P / d h$, are called the indentation stiffness. The hardness and the Young's modulus are derived from these quantities. 


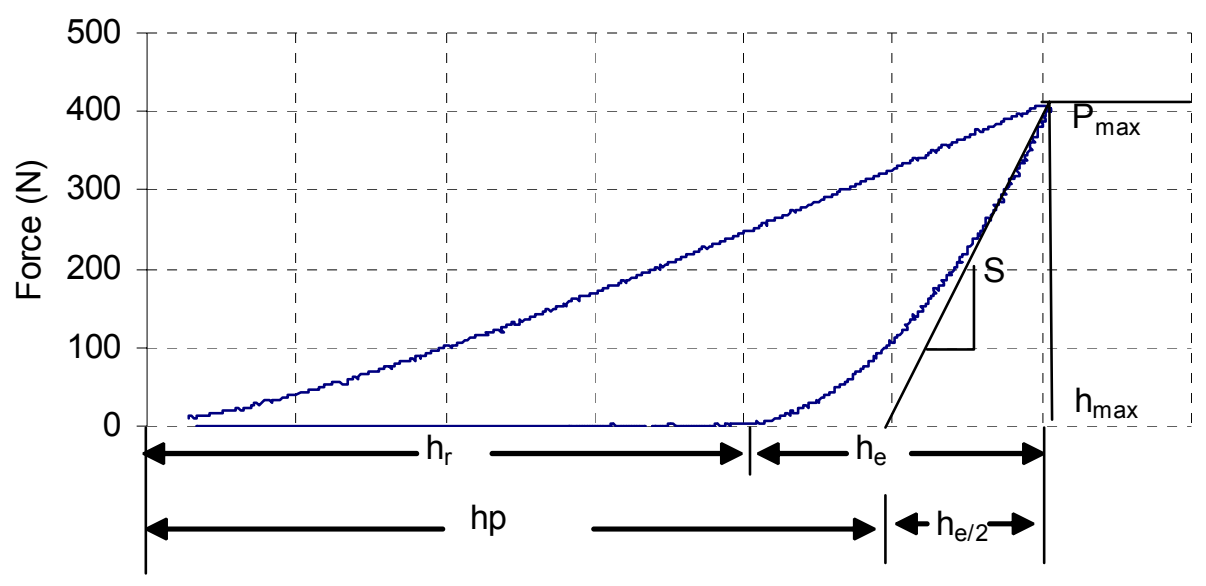

Figure 2.4 Load versus displacement curve (Also called Compliance curve). Experimental data from TIM instrument. Material Aluminum 7075-T6. Indenter sapphire $1.5 \mathrm{~mm}$ diameter.

The fundamental relations for hardness and the reduced modulus are given by [20].

$$
\begin{aligned}
& H=\frac{P}{A} \\
& E^{*}=\frac{\sqrt{\pi}}{2 \beta} \frac{S}{\sqrt{A}}
\end{aligned}
$$

where

$\mathrm{P}=\mathrm{Load}$

$\mathrm{A}=$ Projected area of contact

$\beta=$ Constant depending on the geometry of the indenter

$\mathrm{S}=$ Contact stiffness

This definition of hardness should not be confused with the traditional definition of hardness. In the traditional definition of hardness, the area is calculated with the projected area of 
contact of the residual impression. The traditional definition produces wrong results when the indentation is in the elastic range, giving infinite values of hardness.

The $E^{*}$, reduced modulus, was defined by Tabor [12] as:

$$
\frac{1}{\mathrm{E}^{*}}=\frac{1-v^{2}}{\mathrm{E}}+\frac{1-v^{\prime 2}}{\mathrm{E}^{\prime}}
$$

To determine the contact area, the most widely used method was established by Oliver and Pharr [13]. This method begins fitting the unloading portion of the load-displacement data to the power law relation given by [13]:

$$
\begin{aligned}
& \mathrm{P}=\mathrm{B}\left(\mathrm{h}-\mathrm{h}_{\mathrm{f}}\right)^{\mathrm{m}} \\
& \text { where } \\
& \mathrm{B} \text { and } \mathrm{m} \text { are empirical fitting parameters } \\
& \mathrm{h}_{\mathrm{f}}=\text { Is the final displacement after completing the cycle. }
\end{aligned}
$$

The contact stiffness (S) is established by analytically differentiating the Equation (2.37) and evaluating the result at the maximum depth of penetration, $\mathrm{h}=\mathrm{h}_{\max }$, that is:

$$
\begin{aligned}
& \mathrm{S}=\left(\frac{\mathrm{dP}}{\mathrm{dh}}\right)_{\mathrm{h}=\mathrm{h}_{\max }}=\mathrm{Bm}\left(\mathrm{h}_{\text {max }}-\mathrm{h}_{\mathrm{f}}\right)^{\mathrm{m}-1} \\
& \text { where } \\
& \mathrm{h}_{\text {max }}=\text { Maximum depth of penetration }
\end{aligned}
$$

To calculate the contact stiffness, the unloading part of the load-displacement curve is used at maximum load. The percentage of the curve used to calculate the slope, depends on the equipment. Typically ranges from 25 to $50 \%$ are suggested in ref [20]. Other values used by 
existent equipment in the market are on the range of $95 \%$ to $50 \%$ of the unload curve. Others equipment use the $1 / 3$ of the unloaded curve.

The following step in the procedure is to determine the contact depth $\left(h_{c}\right)$, which for elastic contact is less than the total depth of penetration $\left(h_{\max }\right)$. The contact depth is estimated by [20]:

$$
h_{c}=h_{\max }-\varepsilon \frac{P}{S}
$$

where

$\varepsilon=$ Constant depending on the indenter geometry

$\mathrm{P}=\mathrm{Load}$

$\mathrm{S}=$ Contact stiffness

The last step in the calculation of the projected contact area is performed by evaluating an empirically indenter area function $A=f(d)$ at the contact depth $h_{c}$. For a Berkovich indenter, the area function is given by [12]:

$$
\mathrm{A}=\mathrm{f}\left(\mathrm{h}_{\mathrm{c}}\right)=24.5 \mathrm{~h}_{\mathrm{c}}^{2}+\mathrm{C}_{1} \mathrm{~h}_{\mathrm{c}}^{1 / 2}+\mathrm{C}_{2} \mathrm{~h}_{\mathrm{c}}^{1 / 2}+\mathrm{C}_{3} \mathrm{~h}_{\mathrm{c}}^{1 / 4}+. .+\mathrm{C}_{8} \mathrm{~h}_{\mathrm{c}}^{1 / 128}
$$

where

$\mathrm{h}_{\mathrm{c}}=$ Contact depth

$\mathrm{C}_{1}$ to $\mathrm{C}_{8}=$ Constants

The area function relates the cross-sectional area of the indenter (A) to the penetration distance $\left(h_{c}\right)$ measured from its tip. The contact area is a derived quantity, not a measured quantity, and it is the source of uncertainties in the Depth Sensing Indentation Method. 
Figure 2.5 shows the results obtained by a group of researchers in the area of nanotechnology [18]. The objective of the research group was to establish a correlation between the data obtained by spherical indentation and the published data for Aluminum $6061 \mathrm{~T} 6$.

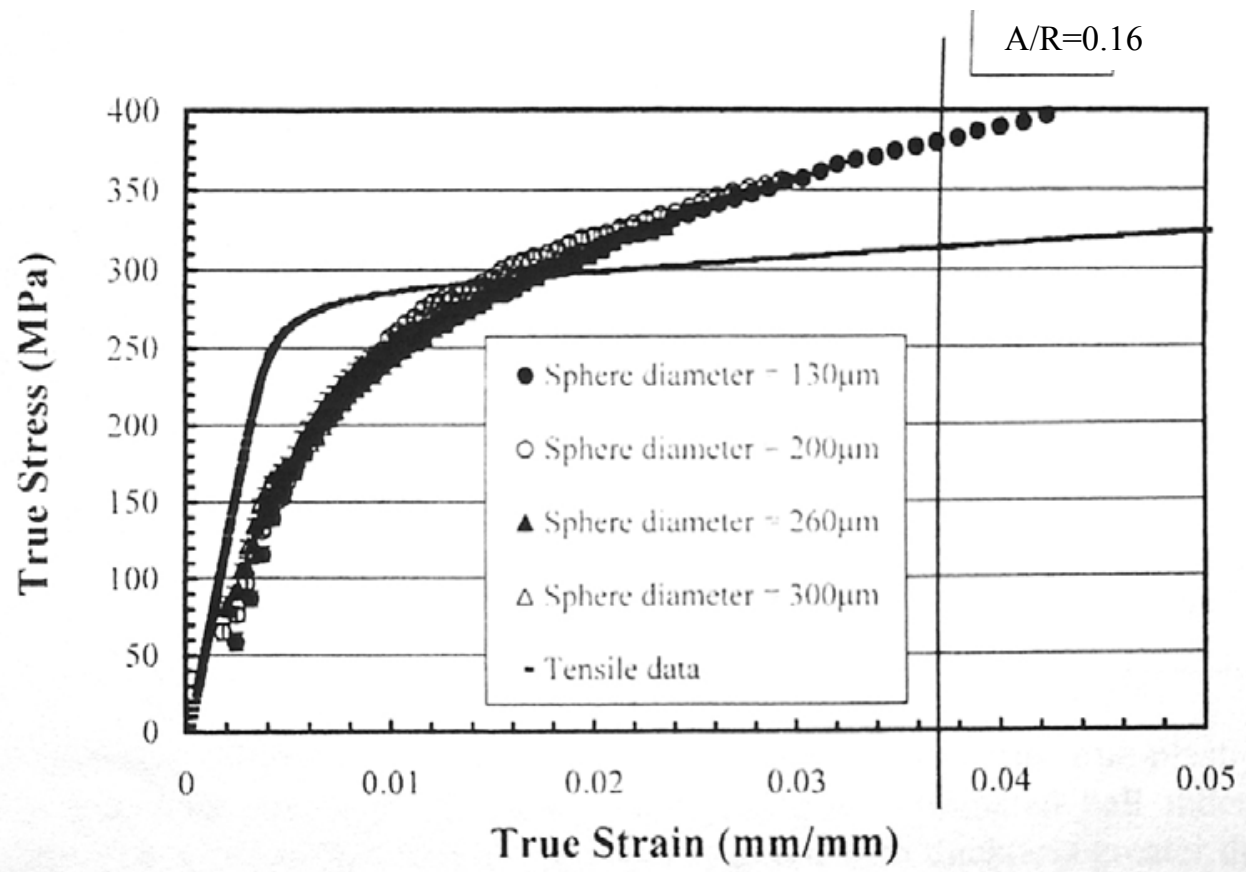

Figure 2.5 True Stress- True-Strain curve for Aluminum 6061-T6 compared to experimental data from depth-sensing indentation method. From [18].

Reference [18] reports a comparison between uniaxial tension test and spherical indentation experiment on aluminum 6061-T6 using the Instrumented Indentation Test. The results show that spherical indentation test can be used to estimate the Young's Modulus and the yield strength, but do not reproduce the shape of the uniaxial stress-strain curve.

\subsection{Indentation Stress-Strain Response}

The indentation method has been used to determine mechanical properties of materials in which the tension test cannot be applied with satisfactory results. The earliest experiments were devoted to develop methods and procedures to obtain similar relations as the tension stress strain 
curve. The classical work of Tabor in the 1950s has been the reference for all the posterior research on the indentation field [12]. The type of indenter used by Tabor was a hardened ball indenter [13], applied to two types of metals: materials with a well defined yield stress; and metals where there is not a defined yield stress.

Tabor [12] suggested that at large plastic deformation, there will be a "representative" value of the indentation stress, say $Y_{r}$, which can be related to the spherical contact pressure $p_{m}$ by a relation of $\mathrm{p}_{\mathrm{m}}=\mathrm{CY}_{\mathrm{r}}$, where $\mathrm{C}$ is a constant with a value of about 3 . Tabor also suggested that there is a relation between the contact diameter of the impression and the radius of curvature of the same impression $\mathrm{R}(\mathrm{a} / \mathrm{R})$. He also assumed that for all indentations with the same value of $\mathrm{a} / \mathrm{R}$, the amount of deformation would be the same because the strain is dimensionless. He concluded that in general $\mathrm{R}=\mathrm{D}$, where $\mathrm{D}$ is the indenter diameter and defined the indentation strain as:

$$
\varepsilon_{1}=\mathrm{f}(\mathrm{a} / \mathrm{R})
$$

From the relation $\mathrm{p}_{\mathrm{m}}=\mathrm{CY}_{\mathrm{r}}$, the mean pressure depends on $\mathrm{a} / \mathrm{R}$. Tabor defined $\mathrm{p}_{\mathrm{m}}$ as:

$$
\mathrm{p}_{\mathrm{m}}=\frac{\mathrm{P}}{\pi \mathrm{a}^{2}}=\varphi(\mathrm{a} / \mathrm{R})
$$

After a series of experimental work on annealed copper and mild steel, Tabor found that there is a relation between the size of the impression $(\mathrm{a} / \mathrm{R})$ and the percentage of deformation corresponding the $\mathrm{Y}_{\mathrm{e}}$, from the relation $\mathrm{p}_{\mathrm{m}}=\mathrm{CY}_{\mathrm{e}}$. The strain can be approximated as:

$$
\varepsilon \approx 0.2 \frac{\mathrm{a}}{\mathrm{R}}
$$


Tabor [12] concluded that if $\mathrm{p}_{\mathrm{m}}$ is plotted against $\mathrm{a} / \mathrm{R}$, a single monotonic curve for all the loads and the entire ball diameters used can be obtained, and that it is possible to compare the results to the tension stress-strain curve.

As for a linear elastic response, a straight-line relationship for these normalized quantities is predicted from the Hertz relation:

$$
\mathrm{p}_{\mathrm{m}}=\left[\frac{3 \mathrm{E}}{4 \pi \mathrm{k}}\right] \frac{\mathrm{a}}{\mathrm{R}}
$$

\subsection{Experimental method to obtain the indentation stress-strain curve}

Using the Tabor's relations, Fisher-Kripps [7] presented a method to obtain the stress/strain curve. The method measures the indentation stress and indentation strain by recording the indenter load and measuring the corresponding diameters of the residual impressions of the polished specimens coated with a thin gold layer. This is used to make it easier to read the diameter using an optical microscope. The method has been implemented into a worksheet that is easy to implement [7]. The following equations are used to calculate the load [7]

$$
\begin{aligned}
& \mathrm{p}_{\mathrm{m}}=\frac{4}{3} \mathrm{E}\left(\frac{1}{\left(1-v^{2}\right) \mathrm{k} \pi} \frac{\mathrm{a}}{\mathrm{R}}\right) \\
& \mathrm{k}=\frac{9}{16}\left[\left(1-v^{2}\right)+\left(1-v^{\prime 2}\right) \frac{\mathrm{E}}{\mathrm{E}^{\prime}}\right] \\
& \mathrm{p}_{\text {suggested }}=\mathrm{p}_{\mathrm{m}} \pi\left(\frac{\mathrm{a}}{\mathrm{R}} \mathrm{R}\right)^{2}
\end{aligned}
$$


Figure 2.6 shows the stress-strain curve obtained by the implementation of the procedure proposed by Fisher-Cripps [7]. The method works only in the plastic region since it is based on measurement of the residual impression.

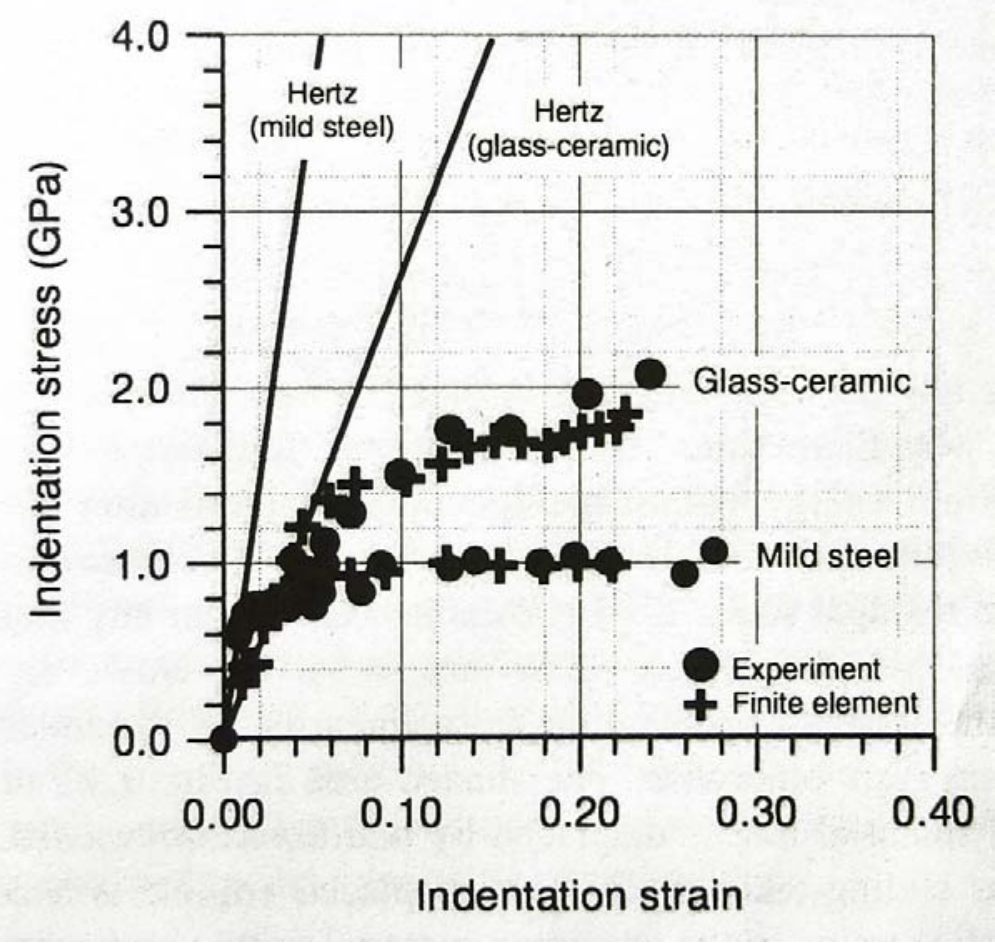

Figure 2.6 Indentation stress-strain results from experimental and Finite Element method. Tungsten Carbide balls of different sizes have been used to obtain the experimental data [7].

Considerable amount of experimental data has been published on the calculation of the modulus of elasticity based on the nanoindentation method using Berkovich indenter. However, only few experimental data are available for Young's modulus evaluation, using spherical indentation [18]. On the other hand, much of the knowledge about the behavior of the spherical indentation has been obtained by finite element simulations $[14,15,16,17]$. 
There is commercial equipment called "Stress-Strain Microprobe ${ }^{\mathrm{R}}$ ". This equipment generates the stress-strain curve of materials, by using a Tungsten Carbide ball indenter with sizes of $0.254 \mathrm{~mm}, 0.508 \mathrm{~mm}, 0.762 \mathrm{~mm}, 1.575 \mathrm{~mm}$ [19]. The characteristics of this equipment are described in the next section.

\subsection{Stress-Strain Microprobe ${ }^{\mathrm{R}}$}

Using spherical indentation, Advanced Technology Corporation has developed a laboratory test instrument to generate stress-strain curve of metallic materials. The technique is called "Automated Ball Indentation (ABI)" and the equipment is called "Stress-Strain Microprobe ${ }^{\mathrm{TM}}$ (SSM)". The procedure performs "strain-controlled" multiple indentations at a single penetration location with a spherical indenter of size in the range of 0.25 to $1.57 \mathrm{~mm}$. The indentation depth is progressively increased with intermediate partial unloading. The technique permits determination of yield strength, stress-strain curve, and the strain-hardening exponent. The specimen surface must be well polished. The following equations are used on the calculation of the stress-strain curve by the SSM instrument [10].

The homogeneous plastic flow portion of the true stress $\left(\sigma_{t}\right)$ - true plastic-strain $\left(\varepsilon_{p}\right)$ curve is represented by the familiar power law equation:

$$
\begin{aligned}
& \sigma_{\mathrm{t}}=\mathrm{k} \varepsilon_{\mathrm{p}}^{\mathrm{n}} \\
& \text { where } \\
& \mathrm{n}=\text { strain-hardening exponent } \\
& \varepsilon_{\mathrm{p}}=\text { true strain. } \\
& \mathrm{k}=\text { strength coefficient }
\end{aligned}
$$


The Stress-Strain Microprobe uses a computer program to solve the following equations to determine the flow curve from the Automated Ball Indenter Test (ABI).

$$
\begin{aligned}
\varepsilon_{\mathrm{P}} & =0.2 \frac{\mathrm{d}_{\mathrm{p}}}{\mathrm{D}} \\
\sigma_{t} & =\frac{4 P}{\pi d_{p}^{2} \delta} \\
d_{p} & =\left(.5 C D\left\{\frac{h_{p}^{2}+0.25 d_{p}^{2}}{h_{p}^{2}+0.25 d_{p}^{2}-h_{p} D}\right\}\right)^{\frac{1}{3}}
\end{aligned}
$$

where

$\mathrm{h}_{\mathrm{p}}=$ plastic indentation depth

$$
C=5.47 \mathrm{P}\left(\frac{1}{\mathrm{E}_{1}}+\frac{1}{\mathrm{E}_{2}}\right)
$$

$$
\begin{aligned}
& \delta=\left\{\begin{array}{lr}
1.12 & \varphi \leq 1 \\
1.12+\tau \ln \varphi & 1 \leq \varphi \leq 27 \\
\delta_{\max } & \varphi \geq 27
\end{array}\right\} \\
& \varphi=\frac{\varepsilon_{\mathrm{p}} \mathrm{E}_{2}}{0.43 \sigma_{\mathrm{t}}} \\
& \delta_{\max }=2.87 \alpha_{\mathrm{m}}
\end{aligned}
$$

where

$\alpha_{\mathrm{m}}=$ Constrain factor index

$\tau=\left(\delta_{\max }-1.12\right) / \ln (27)$

where

$\mathrm{E}_{1}=$ Young's Modulus of the specimen 
$\mathrm{E}_{2}=$ Young's Modulus of the indenter

$\mathrm{D}=$ Indenter diameter

$\mathrm{d}_{\mathrm{p}}=$ Contact diameter

The SSM [19] is a patented instrument, which utilizes an electro-mechanical driven indenter, high-resolution penetration transducer (LVTD) and load cell, a personal computer, 16bit data acquisition/control unit and proprietary ABI software. Figure 2.7 is the true stress-true strain curve obtained by the "Stress-Strain Microprobe" instrument.

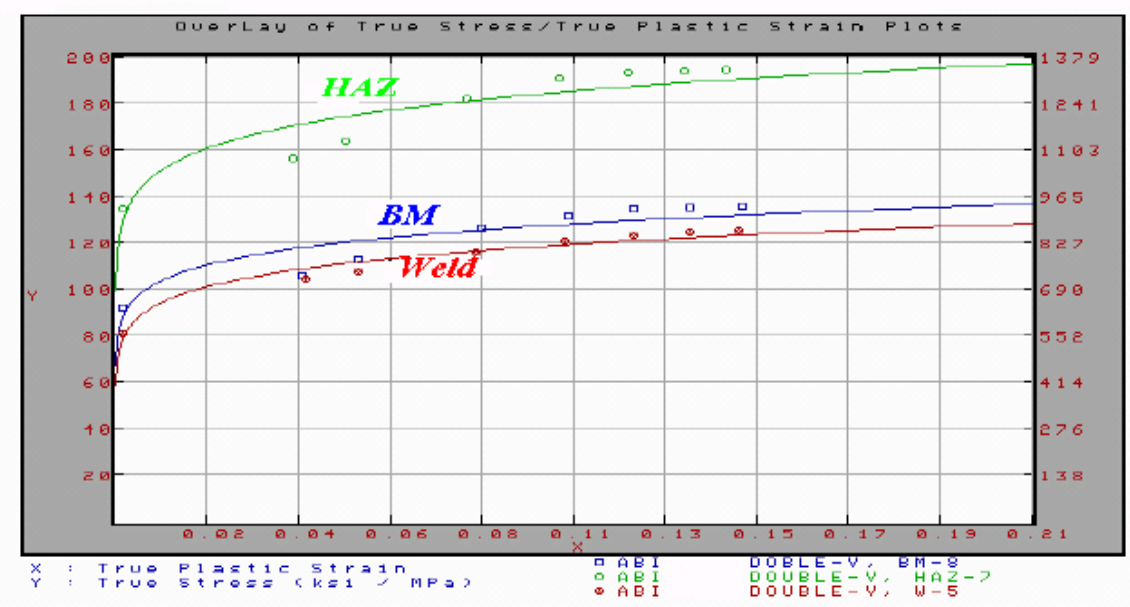

Figure 2.7 True-stress-True-Strain curve obtained from the ABI method, patented equipment "Stress-Strain Microprobe" [19]

\subsection{Through Indenter Measurement (TIM)}

Based on spherical indentation, recently, Dr. Kang's group has developed a Transparent Indenter Measurement (TIM) method [29] for material mechanical property determination.

The operation of the mechanism is as described on reference [29]. Figure 2.8 shows a schematic drawing of the TIM instrument. The TIM instrument uses two kinds of light source, laser light for the interferometry and white light for indentation impression radius measurement. 


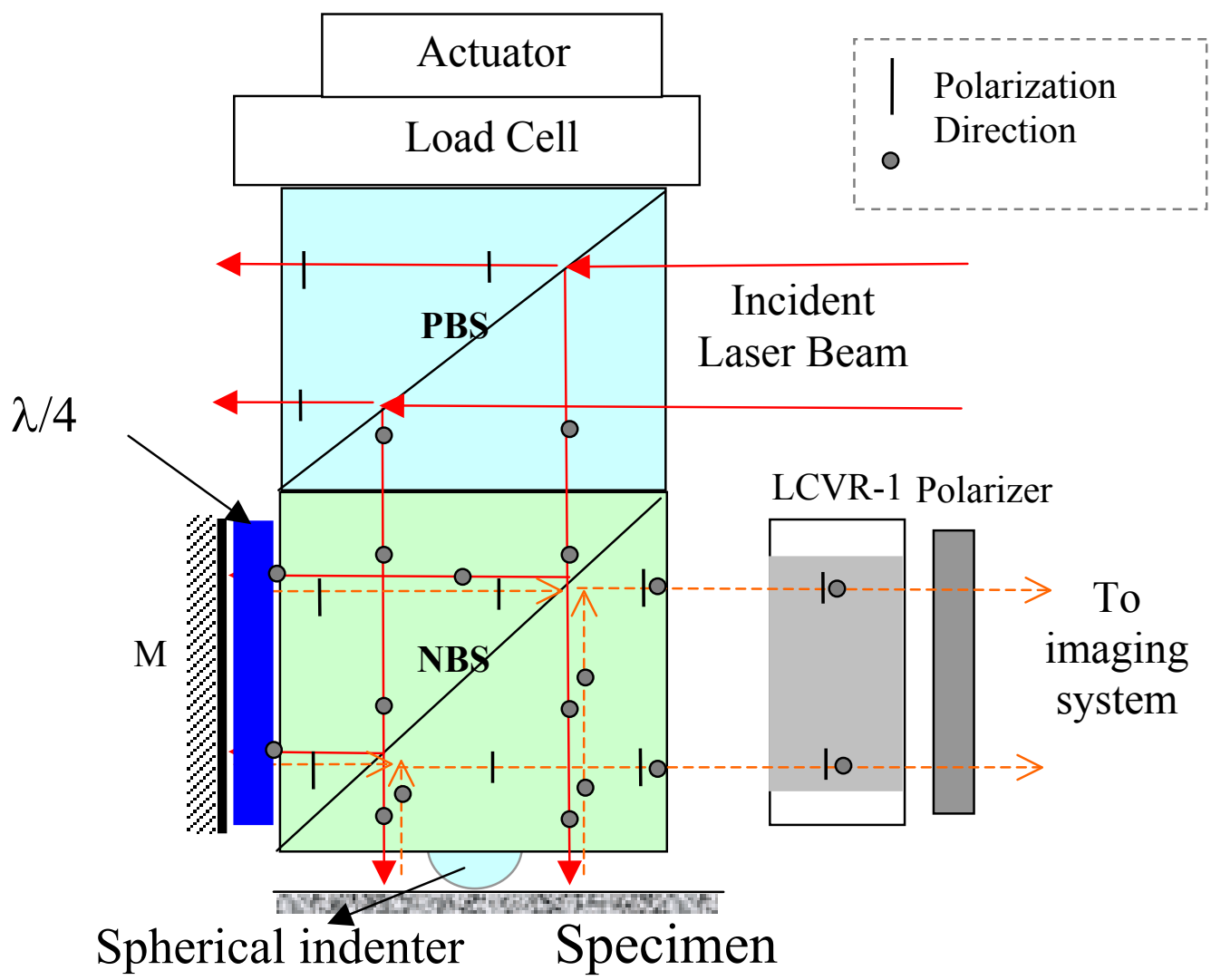

Figure 2. 8 Schematic optical TIM system from Dr. Kang's research group. Figure from [29].

Figure 2.9 is the testing procedures proposed in [29] and involves two steps of loading and unloading. The first cycle of loading is to measure the elastic modulus of the material and the second is to determine the material yield strength and post-yielding stress-strain data. 


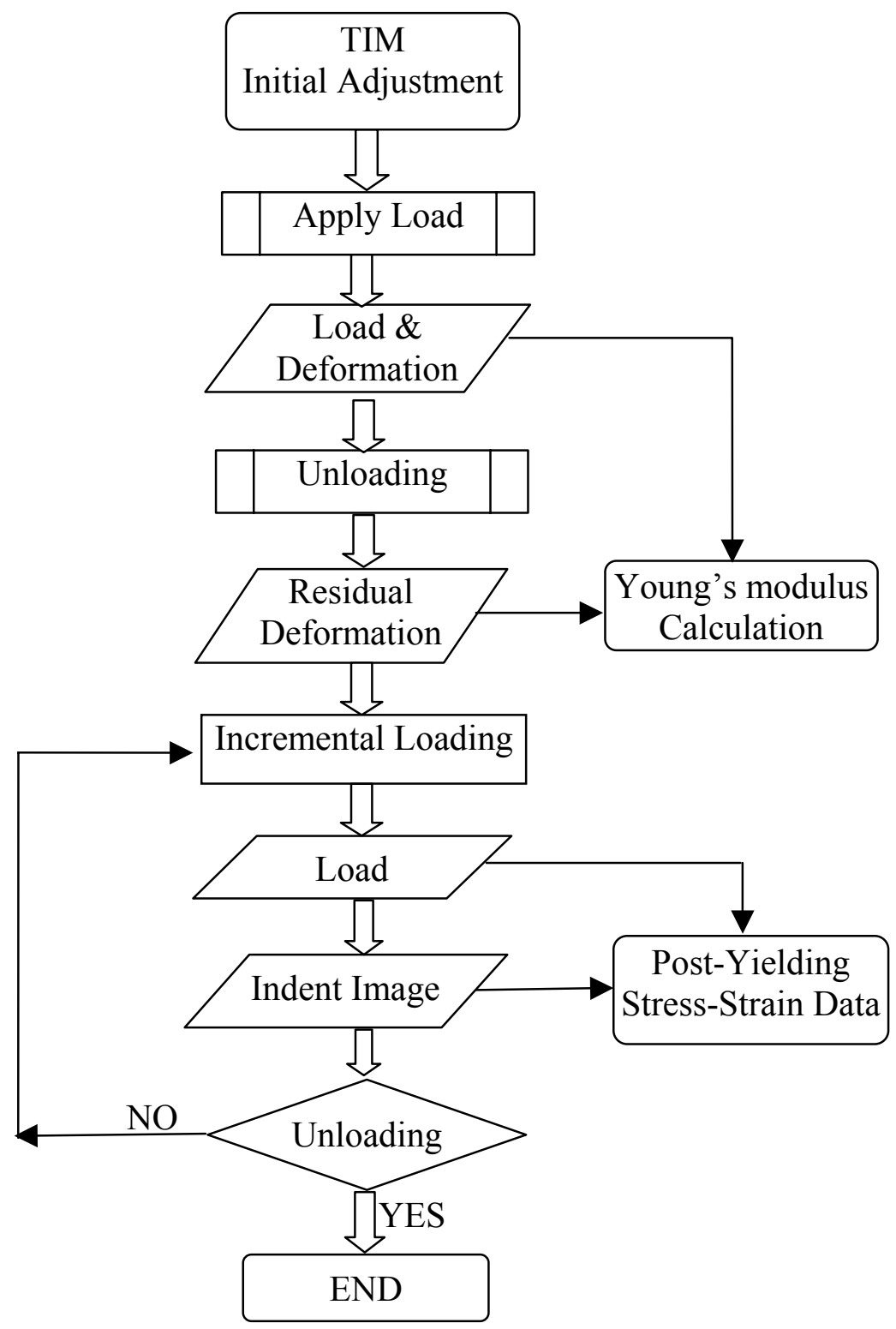

Figure 2.9 TIM testing procedure proposed by Dr. Kang's research group. Graph from [29]

The testing procedure is as follow [29]: In the first step the instrument is adjusted to position and the quality of the image is set-up. At the second step the TIM instrument is used to acquire the initial fringe pattern, without load. The load is applied at the third step. At the fourth step the TIM instrument is used to acquire the out-of-plane fringe pattern. The fifth step 
corresponds to unload the sample. And at the sixth step the data is analyzed to obtain the mechanical properties of the material under test [29]

\subsection{Newton's Rings}

The components of the transparent indenter are a glass (BK7) rod of $5 \mathrm{~mm}$ in diameter and $2 \mathrm{~mm}$ in height and a sapphire half ball glued to the rod $1.5 \mathrm{~mm}$ in diameter, and the specimens are polished to $0.1 \mu \mathrm{m}$ diamond solution, that produce a like mirror surface. These are the ideal conditions for the Newton's rings formation as shown in Figure 2.11. Figure 2.10 shows the experimental arrangement for Newton's Rings formation.

When the transparent indenter with its spherical convex surface is placed on a flat surface of the tested material, at the point of contact, the gap is zero and no air gap in between the two objects in contact. The air gap increases gradually in thickness outward from the point of contact. Let monochromatic light passes through the transparent indenter, the air gap and is reflected back through the transparent indenter, alternating bright and dark concentric rings that are seen around the point of contact will be observed. These concentric rings are due to the interference between the light waves. If a light ray is incident on the upper surface of the lens, the light is reflected and refracted. When the refracted ray strikes the surface, it undergoes a phase change of $\pi$ radians upon reflection. Interference occurs between the reflected and refracted waves, which interfere constructively if the path difference between the light rays is $(m+1 / 2) \lambda$ and destructively if path difference between them is $m \lambda$, where $m$ is order of refraction and $\lambda$ is the light wavelength [30]. Figure 2.11, shows the two quantities of interest for us that are the radius of the $\mathrm{n}$ ring (ring radius) and the distance from the surface to the sphere surface (t). The equations that relate these distances and the wavelength used in this research are [30]: 
$t=\left(\frac{1}{2}\right)\left(m+\frac{1}{2}\right) \lambda$

$r_{n}=\sqrt{\left(R \lambda\left(m+\frac{1}{2}\right)\right)}$

Where

$\mathrm{t}=$ Thickness of the air film

$\mathrm{m}=$ Refraction order

$\lambda=$ Wave length of the light

$\mathrm{R}=$ Radius of curvature of lens

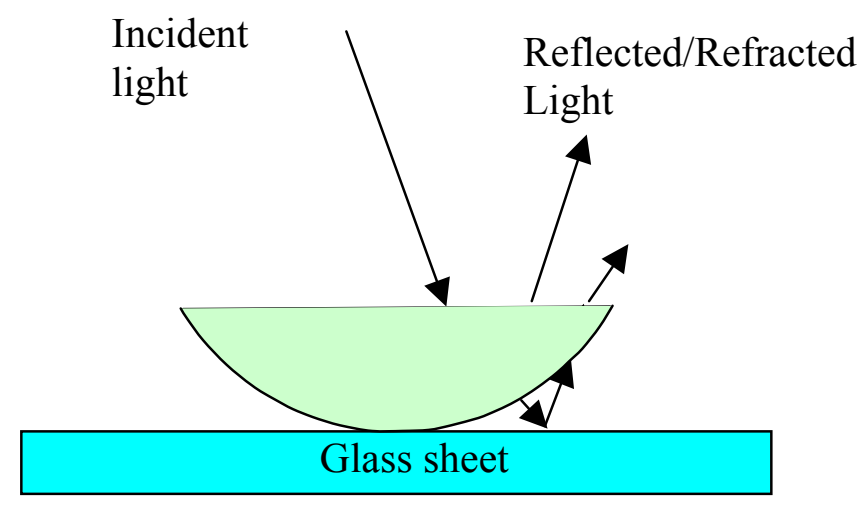

Figure 2.10 Experimental arrangement to generate the Newton's Rings. Adapted from [30]. 


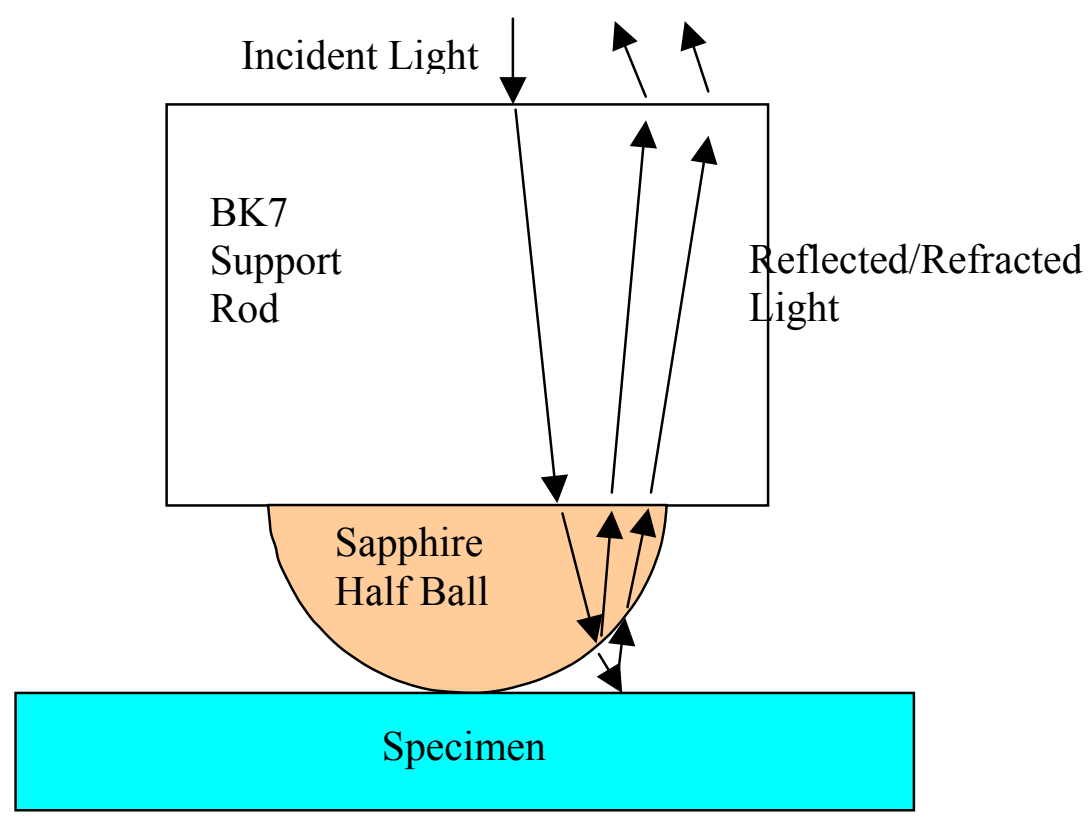

Figure 2.11 TIM experimental set-up, showing the sapphire transparent half ball tip indenter.

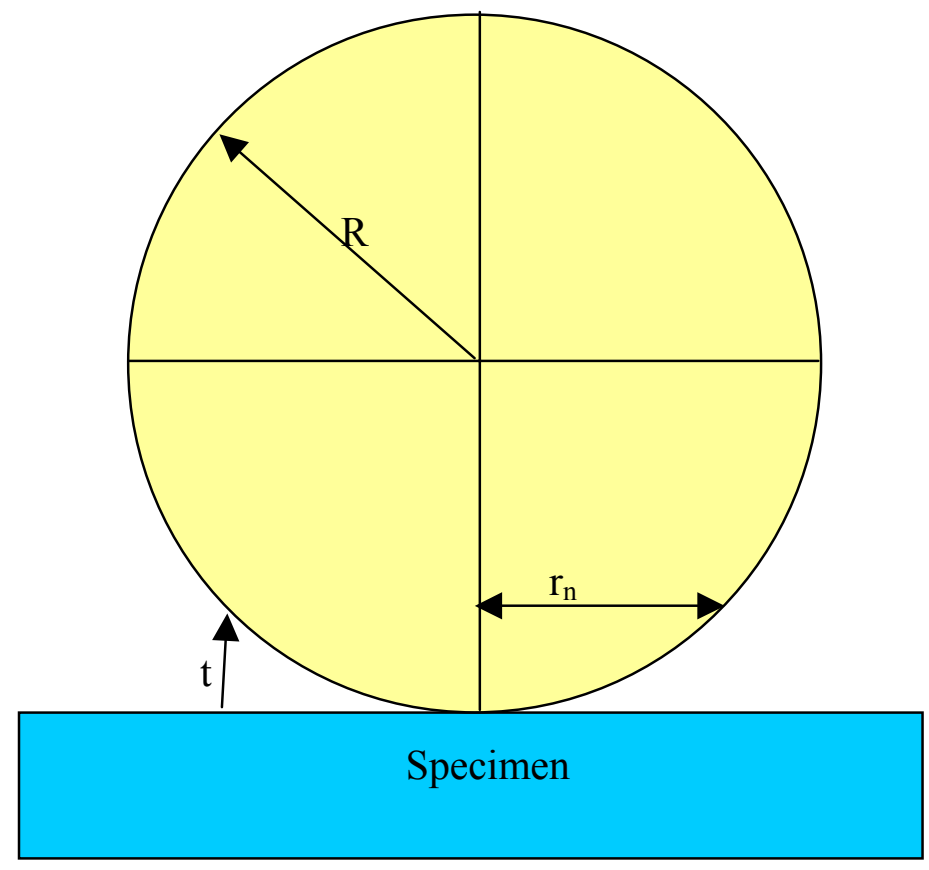

Figure 2.12 Schematic to identify the Newton's Ring radius $r_{n}$, and the thickness of the air film $t$. 


\section{Chapter 3 SIMPLIFIED TIM INSTRUMENT}

\subsection{Introduction}

In this chapter, the characteristics of the traditional material testing equipment will be discussed. The proposed frame and subassemblies are described further in detail. The subassemblies are mechanisms designed for specific functions, i.e., adjust of the position of the indenter normal to the sample. This design strategy adds flexibility to the TIM, and allows using different indenters and loading cells, for different load conditions. Another important mechanism is the microscope support. The function of this mechanism is to assure that the microscope displaces the same distance as the tip indenter. This automates the adjusting of the focus.

\subsection{Tension Compression Machines}

The manufacturers of mechanical testing machines design and build their products in compliance with the requirements of the American Society of Testing of Materials (ASTM) standards or International Standardization Organization (ISO), Deutsches Institut für Normung (DIN), Japanese Standards Association (JIS).

The manufactures differentiate machines according the method used to power the machine. They can be powered either electromechanical or hydraulically. The components of the universal tension-compression machine are: main frame, power device, fixed crosshead, moving crosshead, load cell, extensometer, grips, the data acquisition system and the software to control the movement of the crossheads.

A direct current motor supplies the torque to the precision ball screws, powered an electromechanical machine. The frame crosshead linear motion is due to the rotation of the ball screws. The forces necessary for testing the specimens come from the reaction of the crossheads 
against the base of the system. Typically electromechanical systems are displacement controlled and rarely force controlled. The system capacity varies from $0.5 \mathrm{kN}$ to $600 \mathrm{kN}$ (112 lbf to $135,000 \mathrm{lbf}$ ) and the available models vary from a single column tabletop to dual column high capacity floor models.

The hydraulic machine uses a hydraulic power supply to apply uniaxial or multiaxial loads to the specimen. A closed loop servohydraulic mechanism controls the operation of the machine. This hydraulic machine is force-controlled. The application of the servohydraulic machine is for fatigue testing. The load range varies from $1 \mathrm{~N}$ to $100 \mathrm{MN}$ and the frequency of operation from very low to as high as $200 \mathrm{~Hz}$ or more. High-speed digital electronics provide the right, continuously self-correcting action required to assure controlled testing.

\subsection{Microhardness machines}

Hardness is not recognized as a physical or mechanical property. The measurement of hardness is essentially arbitrary, and it cannot be defined in terms of fundamental units of length, mass or time. In practice hardness is measured in terms of the size of an impression or depth penetration, made on a specimen by an indenter of a defined shape, when a specified force is applied under a normalized time [1].

Hardness standardized calibration blocks manufactured in the United States, Europe, Japan and Australia produce different hardness values. There is no uniformity of values to compare hardness values among different countries. The International Standardization Organization and National Laboratories are working to produce a uniform standard [24] to solve this problem. This is due to the arbitrary nature of hardness, and the Standards are critical in securing uniformity of measurements. Previous to the use of any hardness equipment it is necessary to calibrate the equipment with approved standard blocks. 
Indentation hardness values are given by measuring depth or the area of the indentation using one of over twelve different methods [25]. The existence of these methods is due to the arbitrary nature of hardness over different materials. The hardness tests have a wide application in the industry and research areas. In industry, the hardness method has been applied in plates to built ships, dental ceramics, woods, plastics, rubbers and recently on soft human tissues. Table 3.1 provides an example of the different scales existent for industrial use.

Table 3.1 Example of Hardness methods existent for industrial use, adapted from [25].

\begin{tabular}{|c|c|c|c|c|c|}
\hline Test & Method & $\begin{array}{l}\text { Applied } \\
\text { Force }\end{array}$ & $\begin{array}{c}\text { Indenter } \\
\text { Type }\end{array}$ & $\begin{array}{l}\text { ASTM Test } \\
\text { Method }\end{array}$ & $\begin{array}{l}\text { Measure } \\
\text { Method }\end{array}$ \\
\hline Rockwell & $\begin{array}{l}\text { Rockwell } \\
\text { Standard }\end{array}$ & $\begin{array}{c}60,100,150 \\
\text { kgs. }\end{array}$ & $\begin{array}{c}1 / 16,1 / 8,1 / 4,1 / 2 \\
\text { WC ball, } \\
\text { Diamond cone }\end{array}$ & E18-xx & Depth \\
\hline & Superficial & $15,30,45 \mathrm{kgs}$ & $\begin{array}{l}\text { WC balls and } \\
\text { diamond cone }\end{array}$ & E8-xx & Depth \\
\hline $\begin{array}{c}\text { Rockwell } \\
\text { microhardnes }\end{array}$ & & $\begin{array}{l}500,3000 \\
\text { grams }\end{array}$ & $\begin{array}{c}\text { Truncated } \\
\text { Cone Diamond }\end{array}$ & N/A & Depth \\
\hline Brinell & Optical & $\begin{array}{l}500 \text { to } 3000 \\
\mathrm{kgs}\end{array}$ & $\begin{array}{c}10 \mathrm{~mm} \mathrm{WC} \\
\text { ball }\end{array}$ & E10-xx & Area \\
\hline Shore & Regular & $\begin{array}{c}822(\mathrm{~A}), \\
4550(\mathrm{D}) \\
\text { grams }\end{array}$ & $\begin{array}{l}35^{\circ} \text { Cone (A), } \\
30^{\circ} \text { Cone (D) }\end{array}$ & D 2240 & Depth \\
\hline Microhardness & Vickers & $\begin{array}{l}5 \text { to } 2000 \\
\text { Grams }\end{array}$ & $\begin{array}{l}136^{\circ} \text { Pyramid } \\
\text { Diamond }\end{array}$ & E384-xx & Area \\
\hline Microhardness & Knoop & $\begin{array}{l}5 \text { to } 2000 \\
\text { Grams }\end{array}$ & $\begin{array}{c}1300 \times 1720^{\circ} \\
\text { Diamond }\end{array}$ & E384-xx & Area \\
\hline
\end{tabular}

Hardness testing machines use dead weights to create the operating force. The force can be amplified by means of mechanisms of levers or by a mix of dead weight and hydraulic pressures. Microhardness testers stack the dead weight directly on top of the indenter (10-2000 
gf.). This eliminates the magnification error and other drawbacks, such as knife-edges and hanging weights. Other machines utilize screw drives to apply the force, and load cells to control the amount of force applied [4].

From the point of view of manufacturers, the microhardness equipment presents three problems: accuracy, repeatability, and correlation. The three problems are related to the following causes: machine, operator, environment, sample preparation and calibration [4].

\subsection{Instrumented Indentation Test}

The equipment used for performing instrumented indentation tests consists of three basic components: a) an indenter of specific geometry usually mounted to a rigid column through which the force is transmitted, b) an actuator for applying the force and c) a sensor for measuring the displacement [28]. These three basic components for the Depth Sensing Indentation are similar to those used on the tensile machines. These machines can be adapted for instrumented indentation tests. The depth sensing indentation machines used for calibration purposes are mounted on tensile machine frames $[24,28]$.

The equipments commercially available differ in the ways in which the force is applied and the displacement is measured [27]. Small forces can be generated by the following ways: a) electromagnetically, this is by means of a coil and magnet assembly, introduced by Pethica in 1981 [28], b) electrostatically, using a capacitor with fixed and moving plates, as proposed by Lilleodden et al. in 1995 [28], c) with piezoelectric actuators, actuation through the use of springs. There are different methods that use piezoelectric actuators and cantilever or leaf springs. The magnitudes of the forces are usually inferred from the voltages or currents applied

to the actuators. In piezoelectric driven instruments, a separate load cell is often included to provide a direct measure of the force. 
Displacement is measured by a variety of means including capacitive sensors, linear variable differential transformers (LVDTs), and laser interferometry [28]. The specific device employed that generates the force or displacement determines the range and resolution of the instrument.

\subsection{Development of a simplified TIM Instrument}

The main goal of this research work is to develop simple and reliable indentation equipment that incorporates the TIM method. The main components of the system are: the frame that support the specimen, electronic and optical parts, a closed loop motion generator piezoelectric device, a force sensor or load cell, the optical components i.e. as microscope and camera, stepper motor for fine adjustment of the microscope focus, and the transparent indenter ball that allows the direct measurement of the indentation process and the projected area when the specimen is loaded.

The experimental data and the procedure used to obtain the indentation stress-strain curve discussed in this work have been generated in a laboratory experimental setup. The laboratory experimental fixture consists of an existent tension-compression frame. The frame has a movable crosshead with micrometer resolution. The movable crosshead allows mounting different load cells and indenters of variable diameters to different load conditions and samples of variable size. Another advantage of the movable crosshead is that it has been used to determine the Newton's rings as a method for detecting the contact between the indenter tip and the surface of the specimen.

Some of the system components, such as the piezoelectric actuator, load cell, transparent indenter, and optical setup, have been mounted on the tension compression frame. To control the microscope motion, a $x y z$ micrometric stage is used, in addition to a tilt adapter, used to position 
the microscope normal to the indenter support. The microscope's focus is controlled by a motorized stage.

Figure 3.1 shows a schematic drawing of the simplified TIM developed in this work. The simplified TIM instrument uses only white light to determine the region of contact between the spherical indenter and the surface sample. The drawing also shows the piezoelectric actuator modified with a displacement sensing device serving as a strain gauge. This modification provides displacement close loop control to the actuator. The other mayor parts are the load cell, the adapter that support the mirror, and the sapphire half ball attached to a rod of glass.

The white light is transmitted through the microscope and its direction is changed 90 degrees by the mirror, located at the center of the metal adapter. The white light beam goes through the transparent rod and the half ball indenter, and then reaches the material surface. The same path is followed when the white light comes back after impacting the sample surface, returning to the image system. 


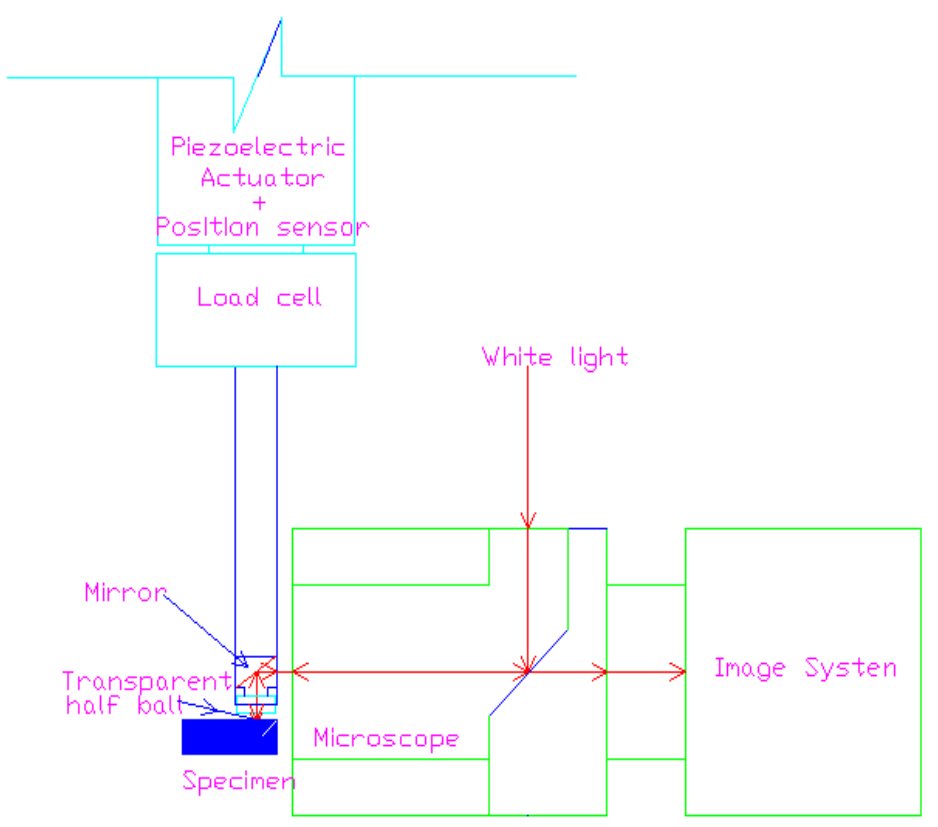

(a)

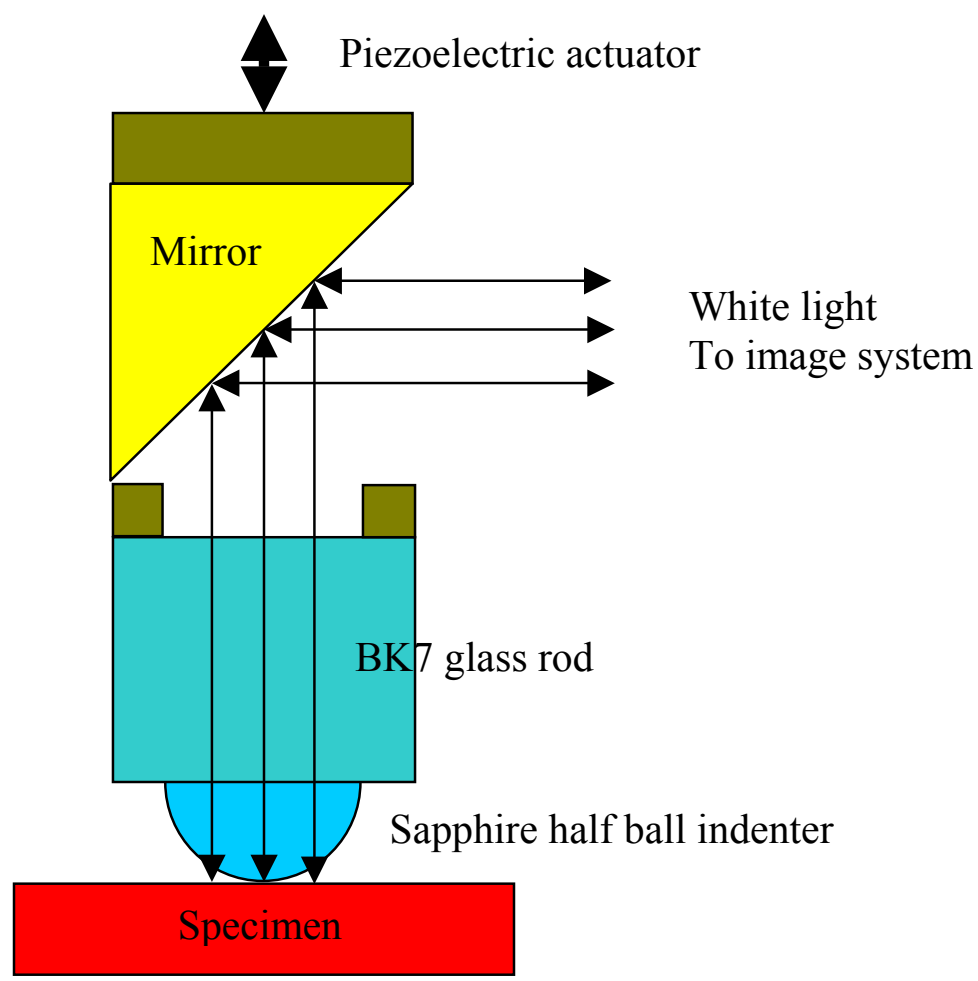

(b)

Figure 3.1 The simplified TIM instrument (a) scaled drawing (b) schematic view. 
Figure 3.2 shows the existent laboratory TIM test fixture from which the results and the procedure for the stress-strain curve generation have been obtained.

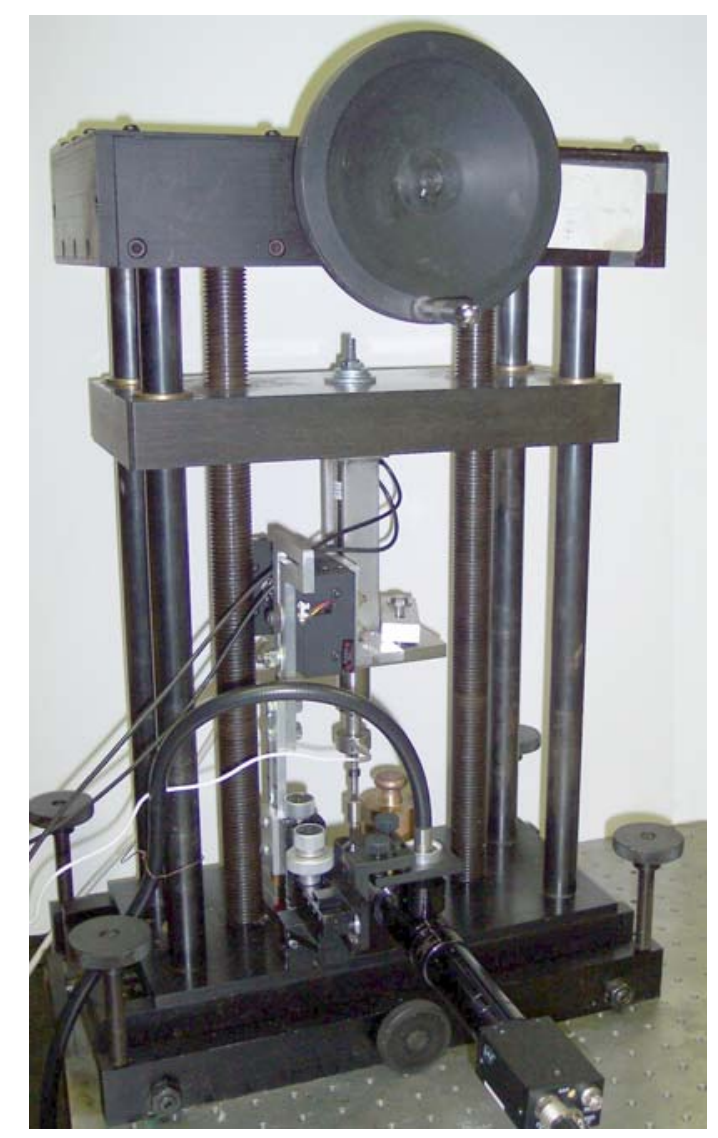

Figure 3.2 Laboratory experimental setup.

The components of the laboratory experimental setup are: 1) frame, 2) stainless steel support for the piezoelectric actuator and stepper motor, 3) steel adapters, 4) load cell, 5) beam splitter, 6) half ball transparent indenter, 7) xyz stage, 8) long working distance microscope, 9) camera, 10) specimen support and 11) specimen.

Figure 3.3 shows the electronic equipment that controls motion of the depth sensing indentation equipment, as well as the white light source, stepper motor control, load cell signal conditioner, and computer that contains the data acquisition card and motion card. The computer 
program that controls the motion and data acquisition of the data and image was programmed in the Labview ${ }^{\mathrm{TM}}$ Software.

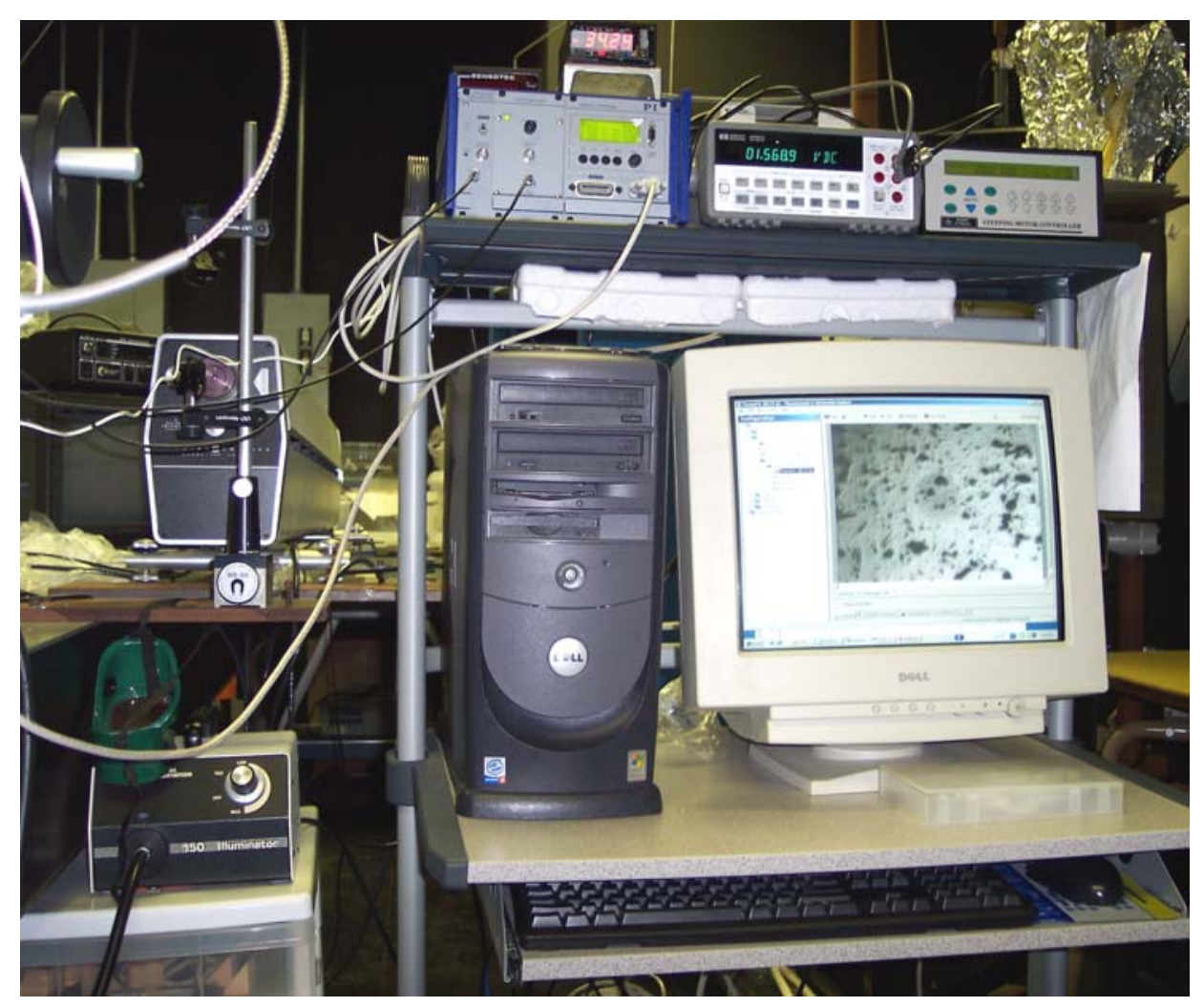

Figure 3.3 Electric, electronic and optical equipment components for the TIM.

Components for the TIM include: 1) high voltage PZT controller, closed loop controller and interface for PZT, 2) load cell signal conditioner, 3) voltmeter, 4) stepper motor controller, white light generator and 5) computer.

Figure 3.4 shows the transparent indenter. A sapphire half ball mounted on a beamsplitter is used as the indenter. Through this mechanism the surface of contact between the specimen surface and the spherical indenter can be measured directly with the use of microscope. The half ball sapphire indenter can have different sizes, thus allowing work over different load ranges. The microscope's magnification can be used to adapt various regimes of load. 


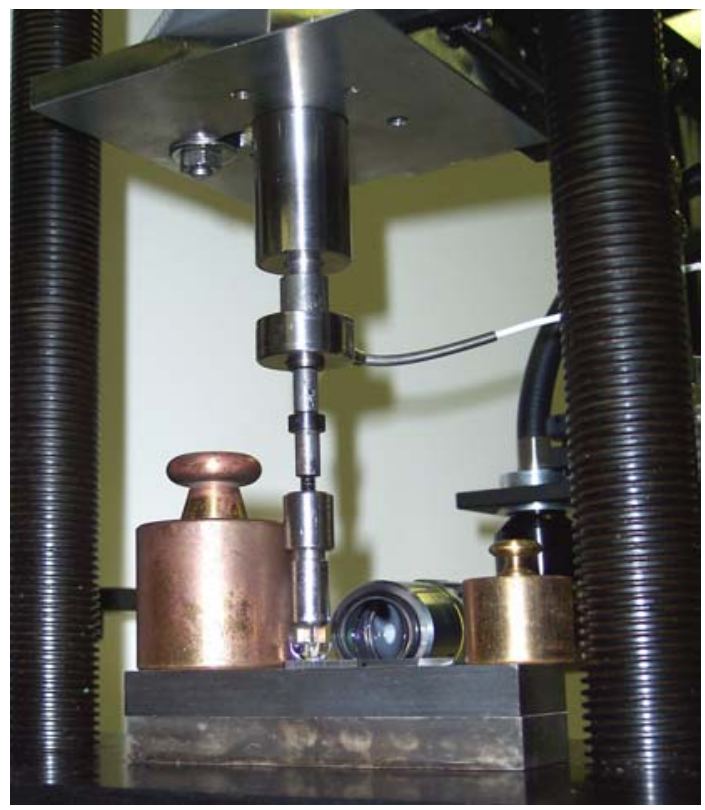

Figure 3.4 Closed-up of the fundamental part of the TIM system.

The white light is generated by a light source and transmitted to the microscope by a fiber optic cable. The white light is diffracted on the beam splitter and reflected by the specimen's surface. If the sample surface is without deformation all the light is reflected to the microscope. When the surface is deformed, not all the light is reflected to the microscope and this condition produces an image of the indentation. The equipment can be easily adapted to different loaddisplacement conditions without the need to buy special adapters.

The manufacture methods used to machine the stainless steel parts of the frame impose severe limitations on the precision required by micrometer resolution equipment. The precision obtained by machining the stainless steel part is in the range of micrometers or less. Another characteristic is that the assembled parts were design to be screwed, for easy mounting and adjusting to different loading conditions. The limitation of this assembly is that there is a reduction in the rigidity of the equipment. The frame must be as rigid as possible in order to avoid deflections of any kind during the indentation process. 


\subsubsection{Frame Design Considerations}

The main constraints, or critical aspects to obtain a precise and accurate mechanical property measurement, as proposed by Herbert [13], are: 1) the correct load-time algorithm; 2) the method to establish the point of contact between the indenter and the sample and 3) the design of the instrument with the appropriate stiffness [13]. These three aspects are considered in this research to design the portable TIM frame and test procedure. The design of the frame is conditioned by the following constrains:

a. The system must be portable.

b. The system must be capable of having a manual adjustment.

c. The frame must accept load cells of different capacities and have interchangeable indenter tips.

d. A compromise between the frame stiffness and portability must be reached.

e. The system must provide a mechanism of indenter alignment normal to the specimen's surface.

\subsubsection{Subsystems description}

The design of the simplified TIM instrument has been done in stages. Each stage corresponds to the design of a subsystem with a specific function. The main subsystem is the mechanism that support and controls the piezoelectric actuator, load cell and transparent indenter tip. The microscope support, the sample support, and the position indenter/sample are the other mechanisms. 


\subsubsection{Mechanism to align the indenter normal to the specimen surface}

The most important feature of portable TIM is the mechanism that allows the indenter tip to be positioned normal to the sample surface. Figure 3.5 shows a schematic diagram of the mechanism. The transparent indenter, the indenter support and load cell are screwed to the piezoelectric actuator, and the piezoelectric actuator is attached to its floating support.

The floating support can rotate about a spherical hardened metallic section, while the tip indenter position is calculated at the center of the sphere. The normal force can be assured to be normal at the point of contact between the indenter and the specimen. The method used to verify the normality, is the presence of concentric Newton rings when the indenter makes physical contact with the specimen surface.

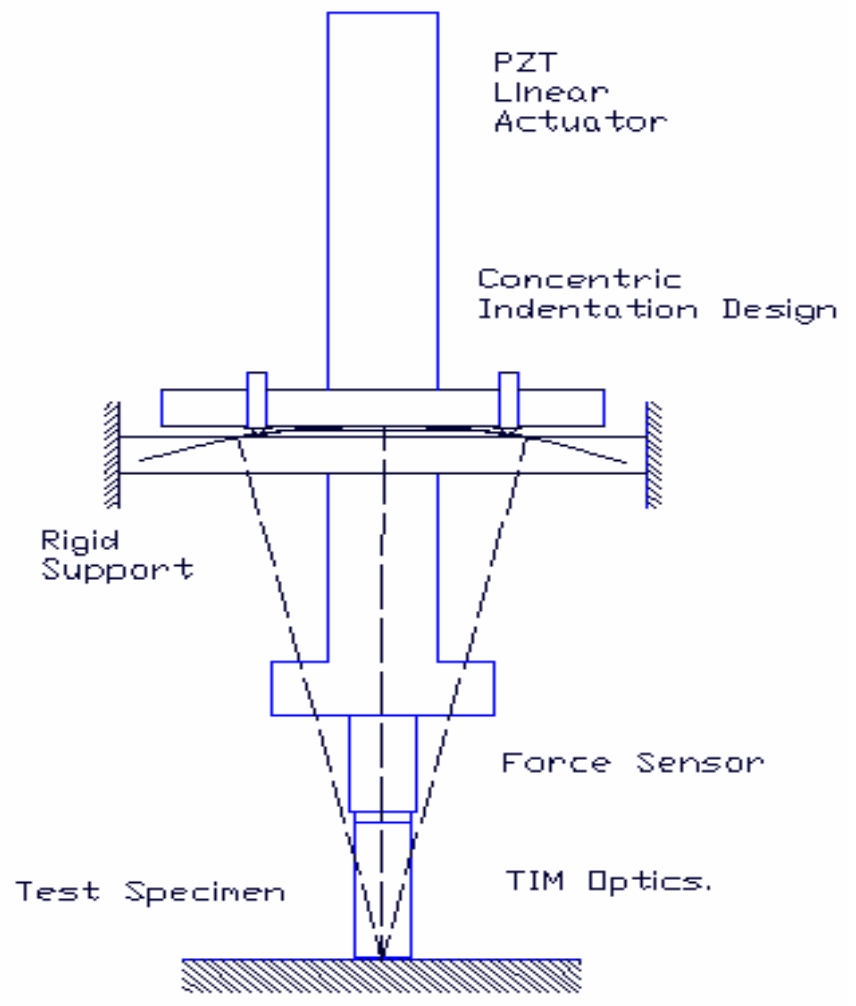

Figure 3.5 Mechanism to position the indenter normal to the specimen. 
The piezoelectric support is floating during the adjusting time of eight plungers. The plungers are located in the following way: four in the base, and four plungers located in the side of the frame. The springs allow the support to be adjusted by the rotation of the ultra fine pitch screws, located on the piezoelectric support. The piezoelectric rotate over a sphere ring section mounted over the base.

Figure 3.6 shows the control position-normal mechanism. Two columns attached to a reference beam support the concentric indentation mechanism. The supporting columns have two slots that allow to the concentric mechanism to be adjusted in height. This is the space that allows the change of different capacity load cells or force sensors. After the concentric mechanism has been positioned normal to the surface, the mechanism is clamped by eight screws.

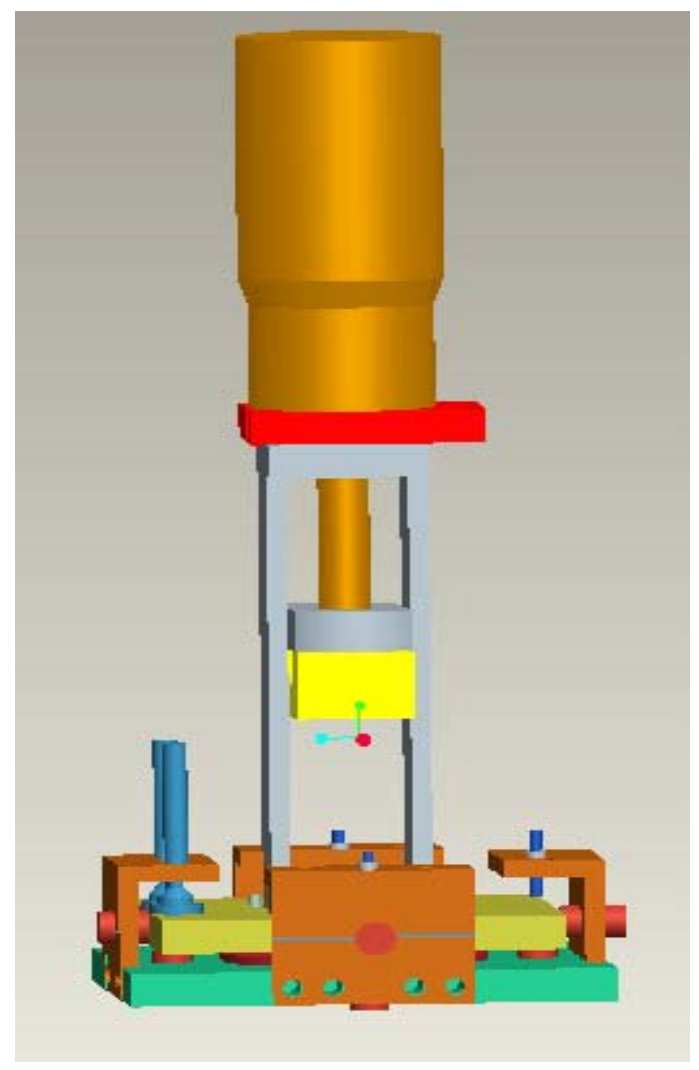

Figure 3. 6 Control mechanism to adjust the rotation of the indenter. The objective is to position the indenter normal to the specimen surface. 


\subsubsection{Removable specimen support anvil}

The anvil shown in Figure 3.7 was designed to support small samples to be tested. The samples are pasted to the anvil with glue and a trapezoidal screw is used for the upward and downward motion of the sample until, the sample is at the indenter position.

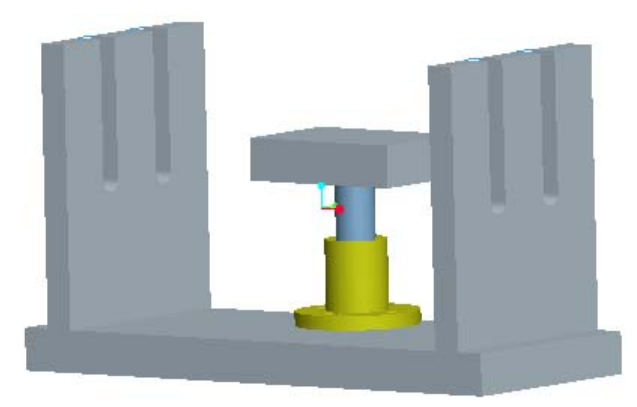

Figure 3.7 Removable simplified TIM instrument base.

The simplified TIM instrument, allows the mounting a dismounting of the base, when used with relative small samples. Rotating the hexagonal screw can move up and down the anvil.

\subsubsection{Mechanism to support the microscope}

The mechanism that supports the microscope is attached to the piezoelectric tip, as shown of Figure 3.8. This mechanism allows the microscope's motion to be the same as the indenter's tip motion. The microscope working distance does not change during the indentation process, which avoid the need of adjustment of the microscope's focus. In this way the indented radius can be measured in a continuously. 
The microscope and the microscope support weight, will induce a bending moment over the piezoelectric crystal that will damage or destroy the piezoelectric crystal (PZT). This dangerous condition is avoided by using a linear bearing, assembled over the case, which allows the bending moment to react over the piezoelectric case and not over the piezoelectric crystal. This feature is illustrated in Figure 3.8.

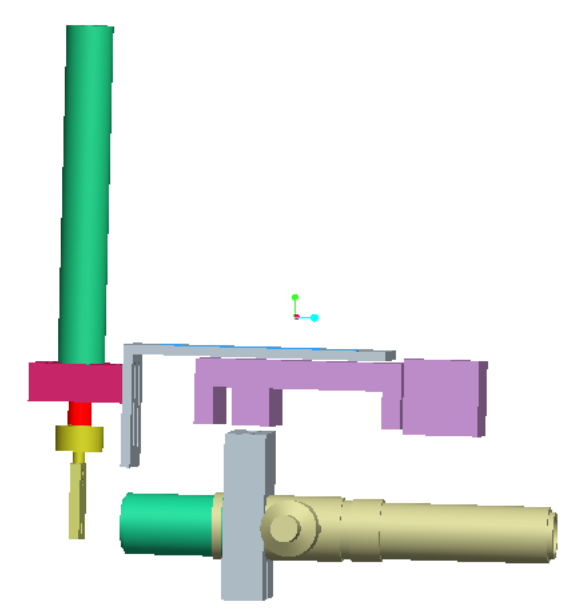

Figure 3.8 Mechanisms to support the microscope.

\subsubsection{Mechanism to control the position of the indenter tip}

The load cells and indenters can be replaced allowing work on the elastic, elastic-plastic or fully plastic regime indentations. An adjustment mechanism must be added to the system to accomplish this function. The adjustment mechanism consists of a Mitutoyo micrometer with a resolution of two microns, a clamp to secure the micrometer, and a bearing to allow the rotation of the screw and fixes the piezoelectric actuator. A diagram of this device is shown in Figure 3.9. 
This advantage has its own risk on the stiffness of the system, because the bearing support is less rigid than a fixed joint.

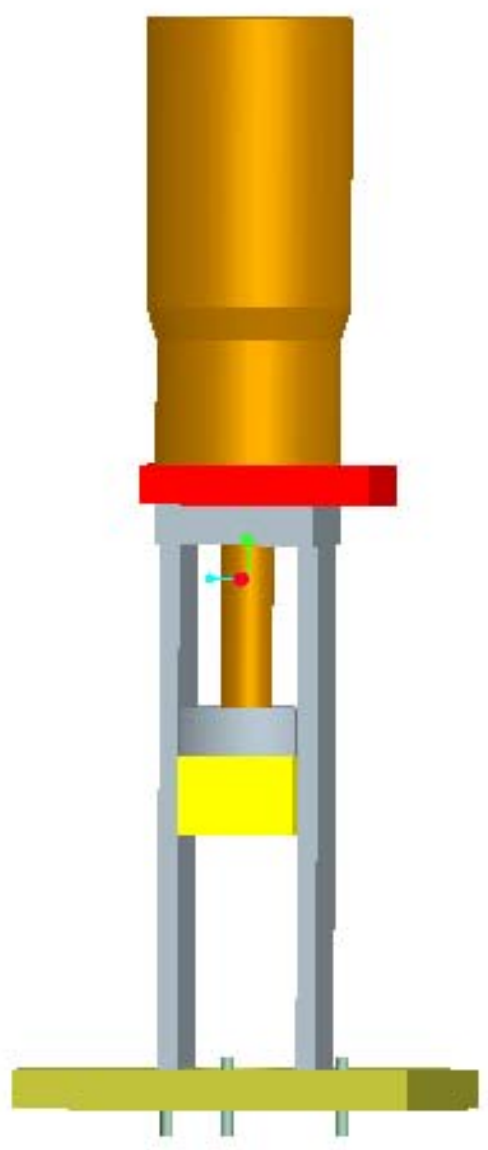

Figure 3.9 Mechanism to control the position of the indenter.

Figure 3.10 shows a $3 \mathrm{D}$ model of the proposed portable TIM system. All the parts have been machined using 304 stainless steel. By changing the base, the frame is adjusted to accommodate different conditions of operation. For example, to round specimens, tubular or non-planar surfaces a cylindrical adapter can be attached. 


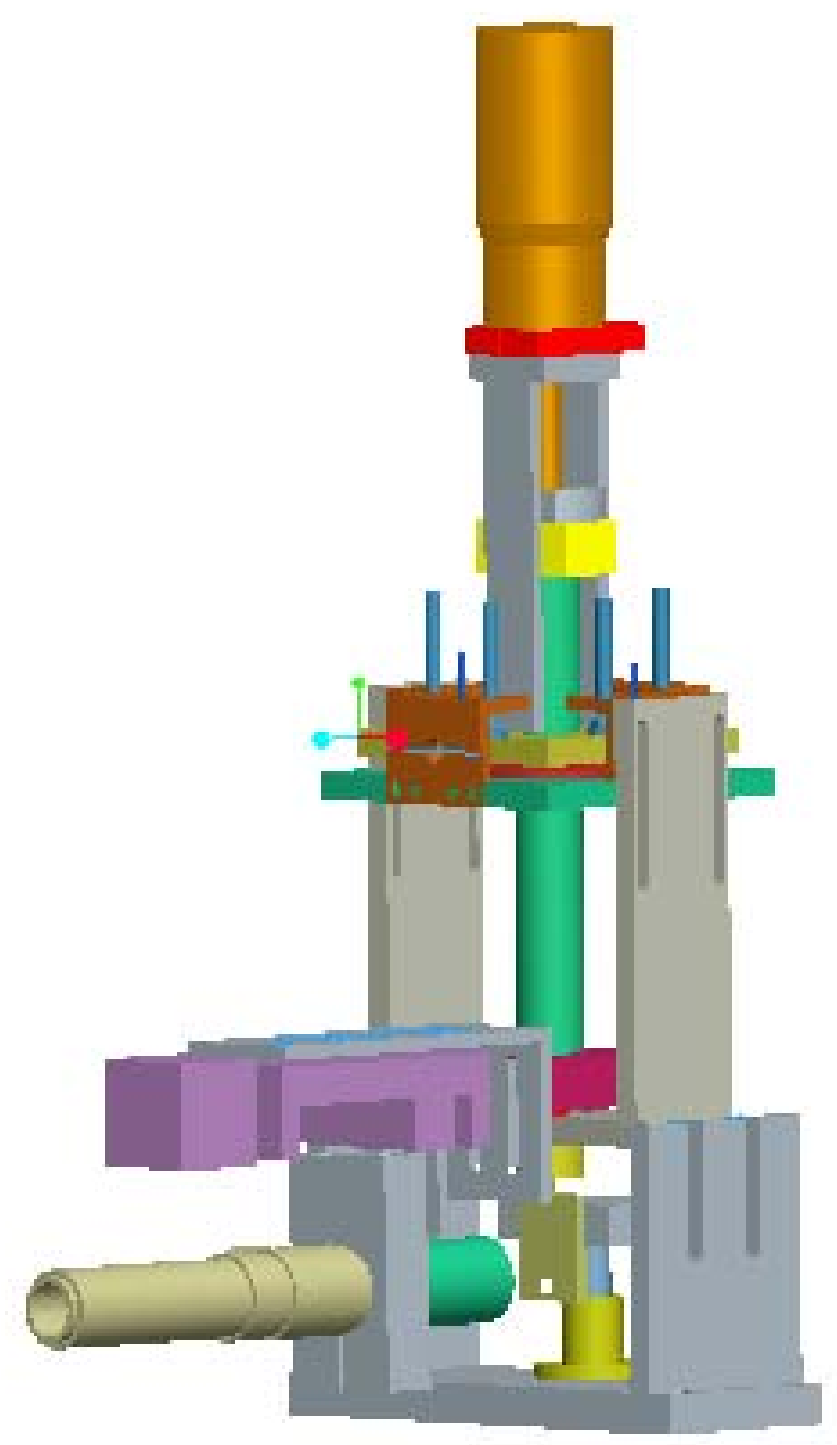

Figure 3.10 A ProEngineer model of the proposed portable TIM equipment.

\subsubsection{Maximum load capacity of the frame}

The main concern in the design process was the stiffness of the instrument. An instrument with flexibility for different load cell looses rigidity due to the positioning mechanism. The stiffness of the frame must be as high as possible, for accurate measurements of depth of penetration, force and contact radius. 
The maximum loading capacity of the frame is limited by the deformation of the adjusting-clamping screw, located at the concentric indentation mechanism. A new design of the mechanism that supports the piezoelectric has been designed. The maximum load with out deformation is about $25 \mathrm{~N}$.

\subsection{Software}

The piezoelectric actuator system includes customized software to control the motion of the actuator, and a strain gauge module that measures the displacement of the crystal tip, which is controlled by a closed loop controller module. A trapezoidal wave is chosen to control the tip piezoelectric motion as shown in Figure 3.11. The hold time for all the cycles has been selected as 15 seconds. This time is the time recommended on microhardness standard [4], and used on nanoindenter equipment by Hysitron ${ }^{\mathrm{TM}}$ and others [9]. The wave shape parameters that define the piezoelectric motion of the tip are:

1. Piezoelectric displacement (in microns)

2. Rising time (milliseconds, seconds)

3. Displacement velocity (microns/second)

4. Duration of the hold period (seconds)

5. Falling time (milliseconds, seconds)

6 . Number of periods to execute the motion. 


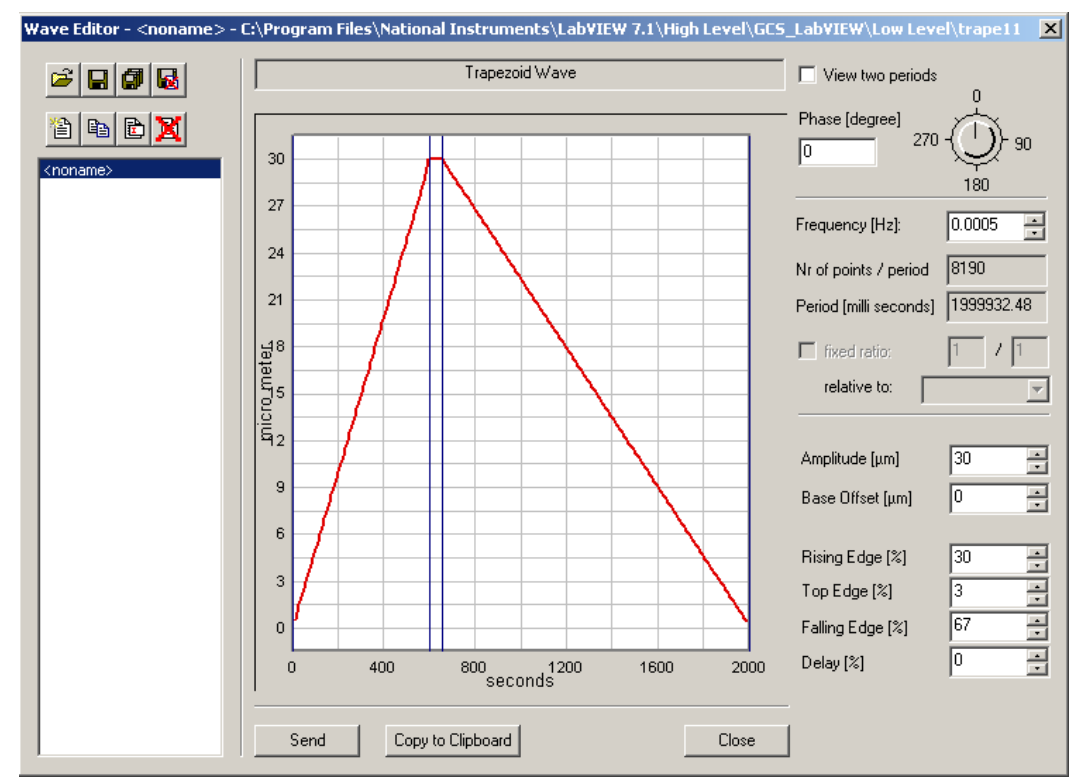

Figure 3.11 Trapezoid wave shape, showing the control parameters

(Window provided by "Physik Instrumente").

Figure 3.11 shows an example of a trapezoidal wave used to control the piezoelectric motion. The velocity used for all indentation is 0.1 micron/second of piezoelectric tip motion.

A Virtual Instrument (VI's) on LabView ${ }^{\mathrm{TM}}$ software provided by Physik Instrumente $^{\mathrm{TM}}$, has been modified to include the force signal from the load cell and the indentation contact image from a camera which is fitted with a long working distance microscope in order to measure the radius of the spherical indentation.

Figures 3.12 and 3.13 show the computer control panel used to control and monitor the function of the simplified TIM instrument, and a portion of the Labview program modified to acquire the load and the signal from the image acquisition subsystem. 


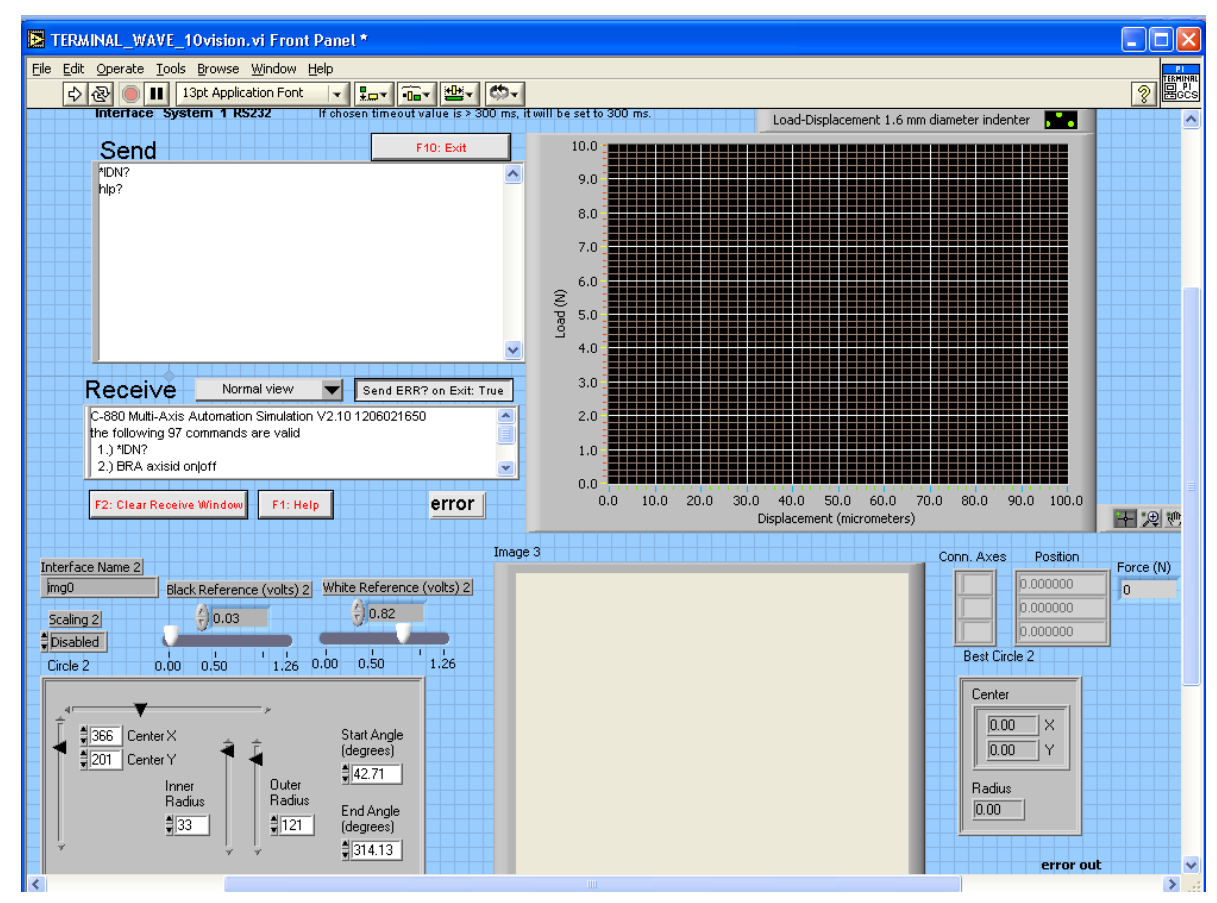

Figure 3.12 Control panel for the piezoelectric actuator, load cell and image acquisition.
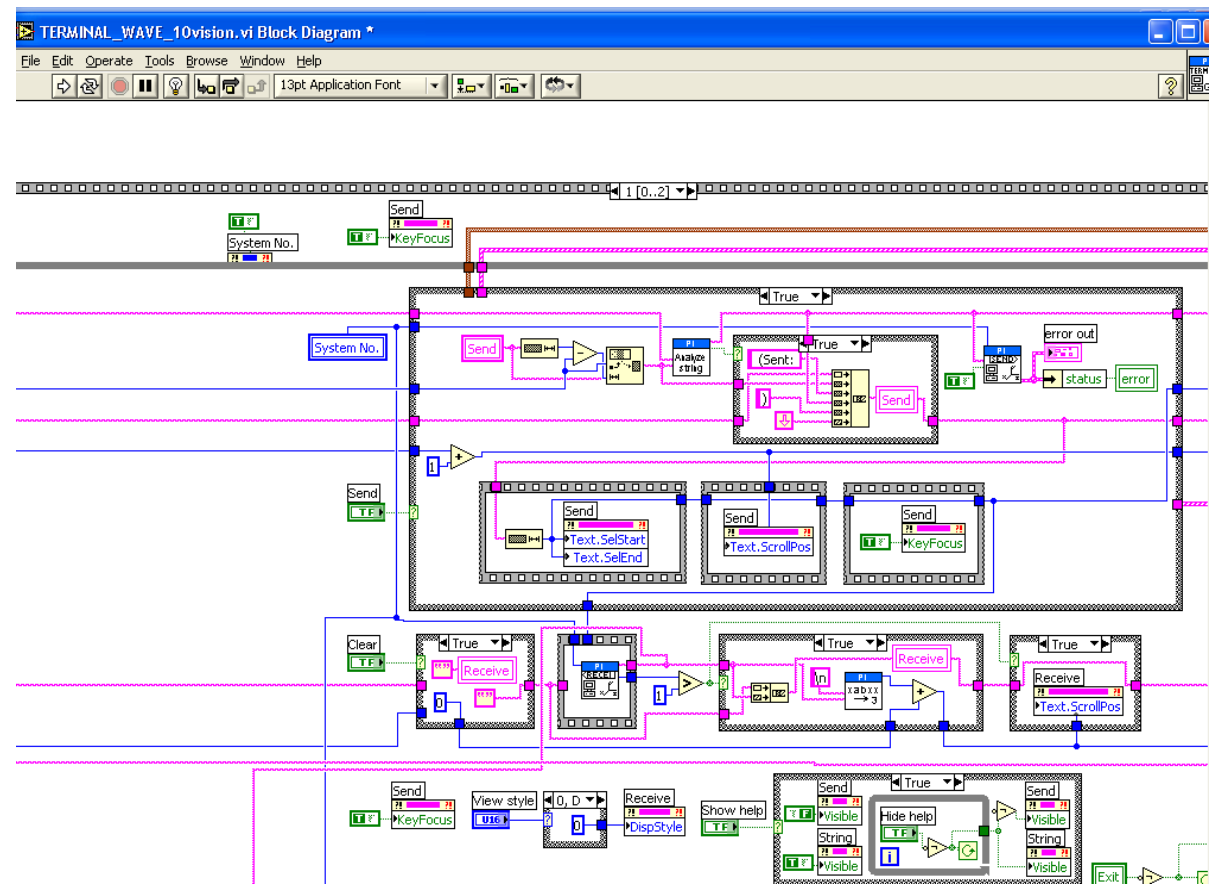

Figure 3.13 Computer program on LabView ${ }^{\mathrm{TM}}$ software. 


\subsection{The Simplified Transparent Indenter Measurement Prototype}

Figure 3.14 and Figure 3.15 show the prototype of the simplified TIM instrument. Figure 3.14 shows the details of the components of the simplified TIM instrument. The frame was manufactured on Stainless Steel 304. The displacement of the piezoelectric actuator is 40 micrometers of displacement. The load cell shown has a capacity of $22.24 \mathrm{~N}$ and the microscope magnification is $10 \mathrm{X}$. Figure 3.15 shows the simplified TIM instrument, the computer interface, the piezoelectric closed loop control and the high voltage piezoelectric controller. The load cell signal conditioner and the voltmeter are also shown.

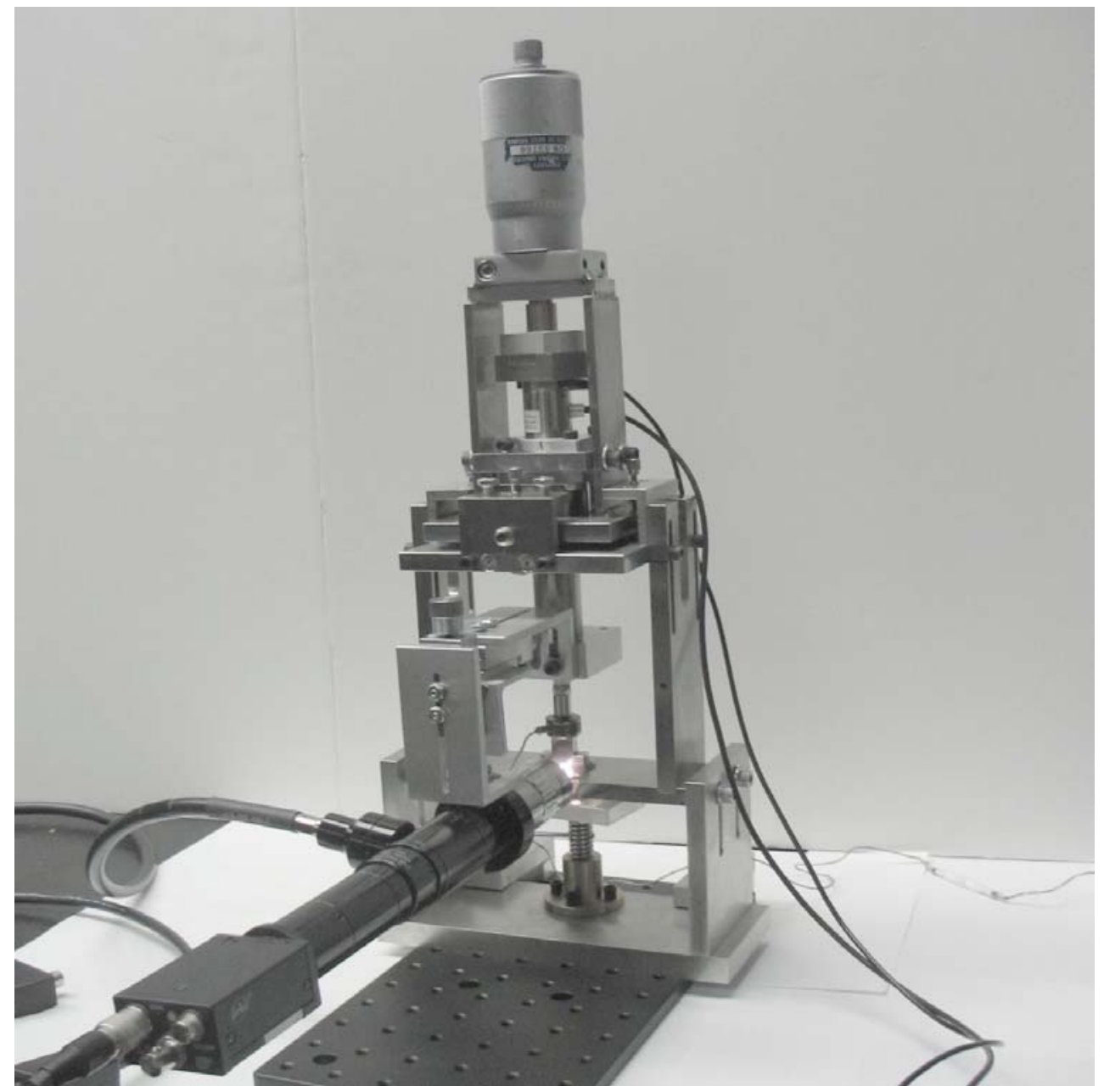

Figure 3.14 The Simplified Transparent Indenter Measurement Prototype. 


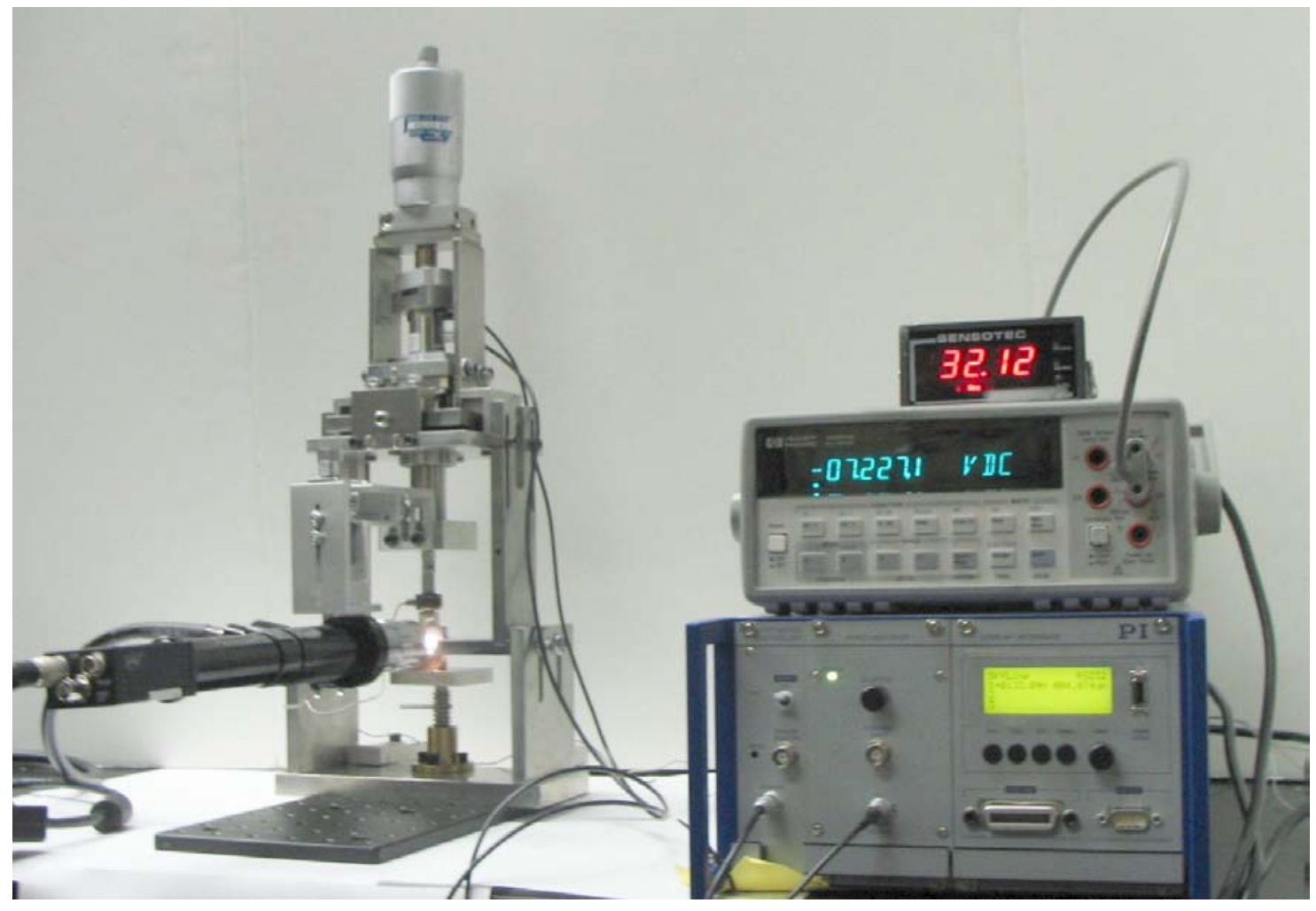

Figure 3.15 The simplified TIM instrument prototype and accompanying electronic components 


\section{Chapter 4 RESULTS}

\subsection{Introduction}

In this chapter the procedure to generate the stress-strain curve using the simplified TIM instrument is outlined. The procedure was developed following models that have been proved to work successfully, such as the models of Hertz, Oliver and Pharr and Tabor [18]. The simplified TIM instrument developed at West Virginia University use the load piezoelectric displacement curve, the applied force and the contact indentation area as experimental data inputs for the indentation models. The mechanical properties of interest are the elastic modulus, the yield strength and the strain-hardening exponent. These three parameters define the three regions of the strain-stress curve: the elastic range, the elastic-plastic region and the fully plastic region.

Figure 4.1 shows the data generated by the simplified TIM instrument for an indentation test. The contact area or residual impression picture is used to calculate the contact radius. The load- piezoelectric displacement curve is processed to obtain the loading system, the contact stiffness and compliance. For example Figure 4.1 shows each set of load-piezoelectric displacements and the indentation images corresponding to: (a) Elastic range of material, applied load $0.182 \mathrm{~N}$, radius of contact 11.6 microns, image magnification, 50X (b) Plastic region, applied load 28.559 N, radius of residual impression 79.2 microns, 10X (c) Fully Plastic region, applied load $70.165 \mathrm{~N}$, radius of residual impression 118.8 microns 10X.

The image of Figure 4.1 (a) is used to derive the elastic modulus of the material. Figures 4.1 (a) and (b) are used to calculate the yield strength and the strain hardening exponent. 

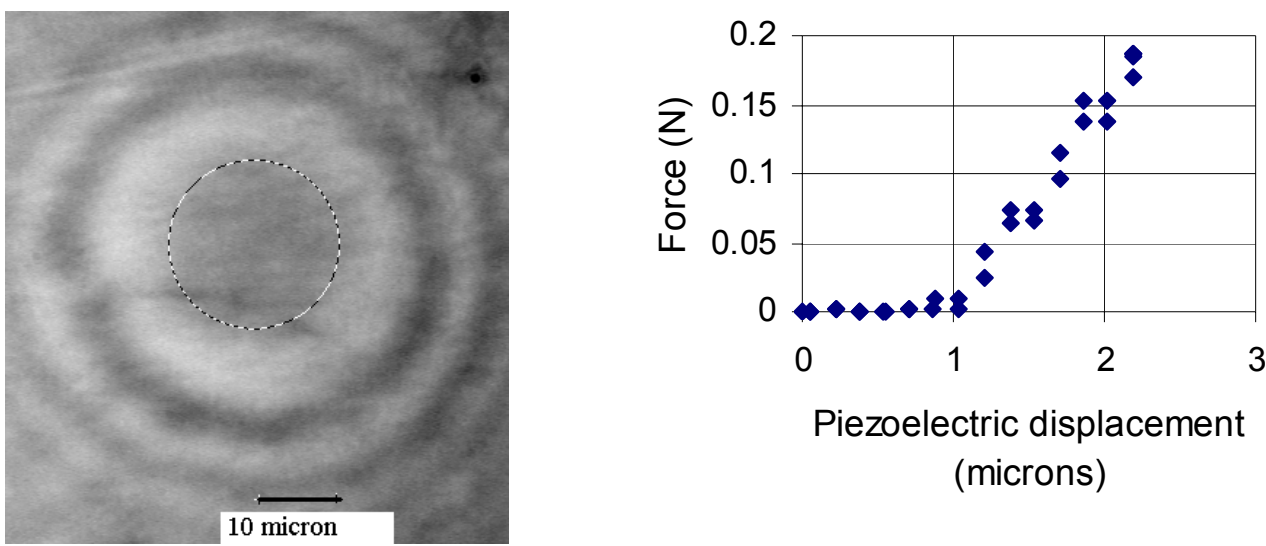

Piezoelectric displacement (microns)

(a)
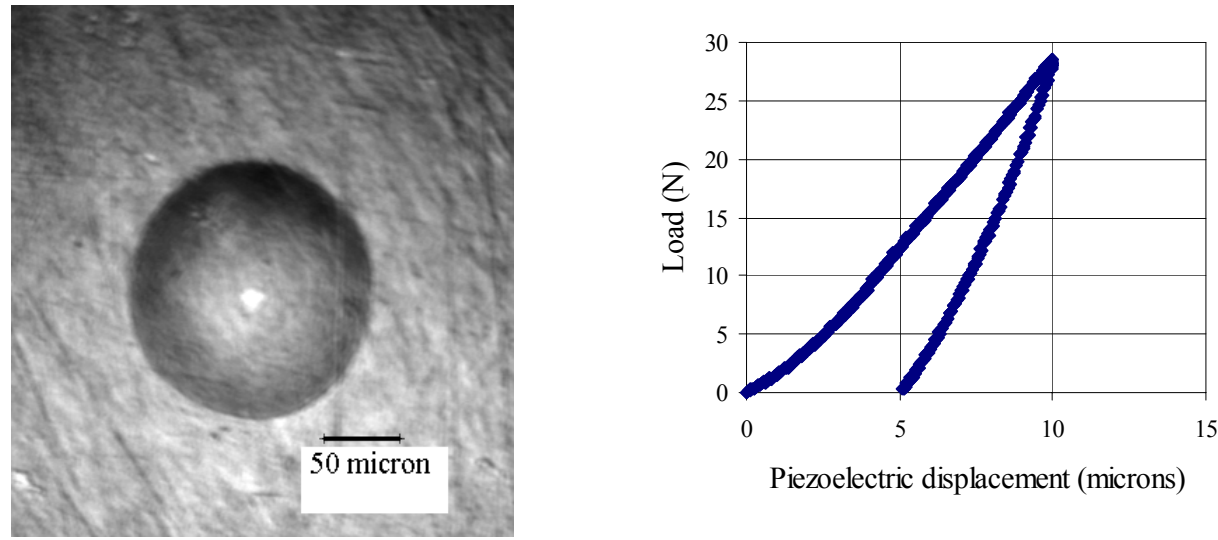

(b)
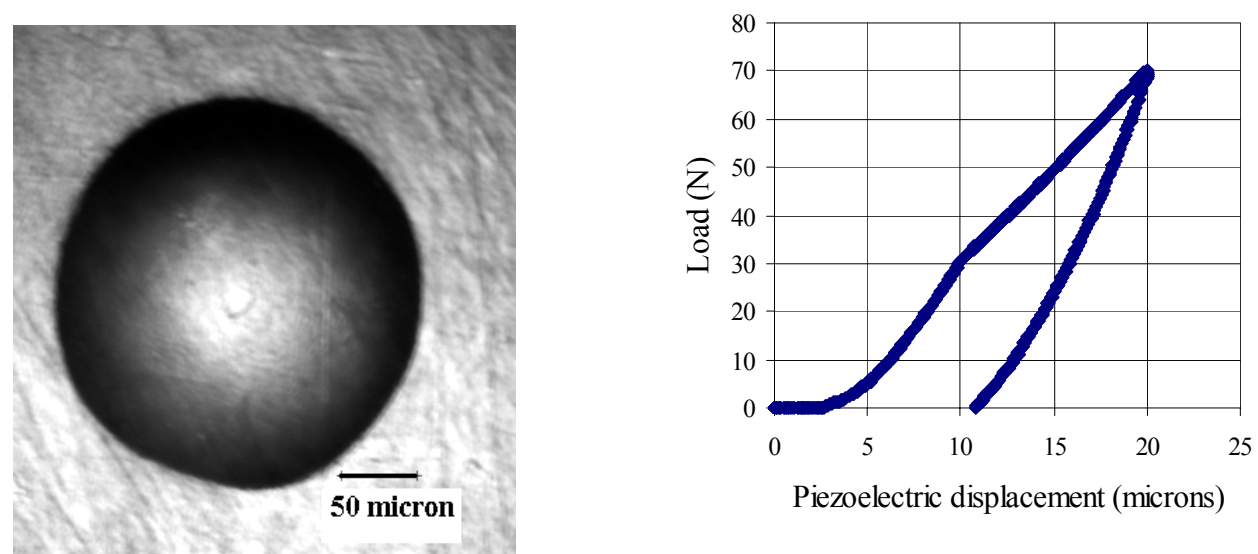

(c)

Figure 4.1 Raw data from the TIM instrument. Aluminum 7075-T6. 


\subsection{Elastic modulus}

The TIM instrument has the capability to determining the elastic modulus by two ways. The first one uses the optical capabilities of the TIM instrument to determine the position of the specimen's surface relative to the indenter position through the Newton's rings and the Hertz' surface model. The second method uses the Oliver and Pharr model which is based on the concept of the instrumented indentation testing.

\subsubsection{Elastic modulus determination based on the Newton's rings}

The images of Newton's rings obtained through the transparent indenter of the simplified TIM instrument are used to calculate the specimen surface position relative to the spherical indenter. The contact radius is calculated by equating the specimen surface position for each Newton's ring radius with a modification of the Hertz's equation that provide the displacement of the specimen surface under a spherical indenter.

The Hertz equation of surface displacement is referenced to the coordinate system through the equation of maximum depth of penetration. The modified equation is only a function of the contact area and does not involve a previous knowledge of the elastic properties of the materials under test. The contact radius used to determine the material's elastic modulus, is the average value of the available number of Newton's rings in the image. For each Newton's ring, a value of contact radius is determined.

The Hertz elastic contact analysis assumptions are applicable to the proposed method following some special considerations:

a) The indenter is spherical and non-deformable. 
b) The relative curvature of the contact area is the same as the curvature of the indenter R' $=$ $\mathrm{R}$, where $\mathrm{R}^{\prime}$ is the relative curvature of the contact area and $\mathrm{R}$ is the radius of the indenter.

c) The coordinate system origin is located at the no load point of contact between the spherical indenter and the flat specimen surface, located at the lower side of the spherical indenter.

The applied force and the Newton's ring radius are the experimental values provided by the TIM instrument, through the load-displacement diagram and the image of the indentation specimen surface as shown in Figure 4.2.

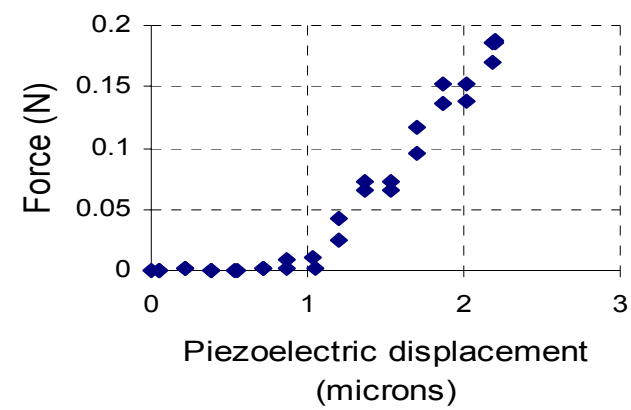

(a)

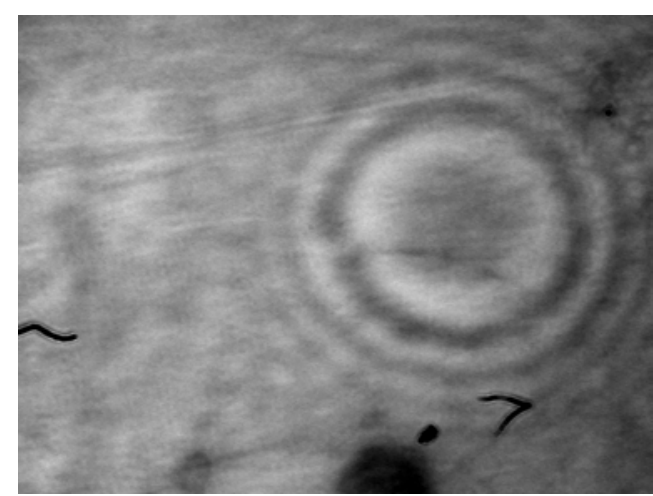

(b)

Figure 4.2 The TIM instrument provides a) The load-piezoelectric tip displacement, and b) the image of the specimen surface with the radius of the Newton's rings.

The proposed method to find the elastic modulus using the Newton's rings is based on geometrical relations established between the indentation surface image and the spherical indenter. The method's equations were derived from Figures 4.3, 4.4 and 4.5.

The coordinate system, spherical indenter position and the original specimen surface are presented in Figure 4.3. The coordinate system provides the position of the specimen surface for 
every Newton's ring radius and does not require a previous knowledge of the maximum indenter penetration depth. The indenter is fixed and the specimen surface is moving up, as the load is increased.

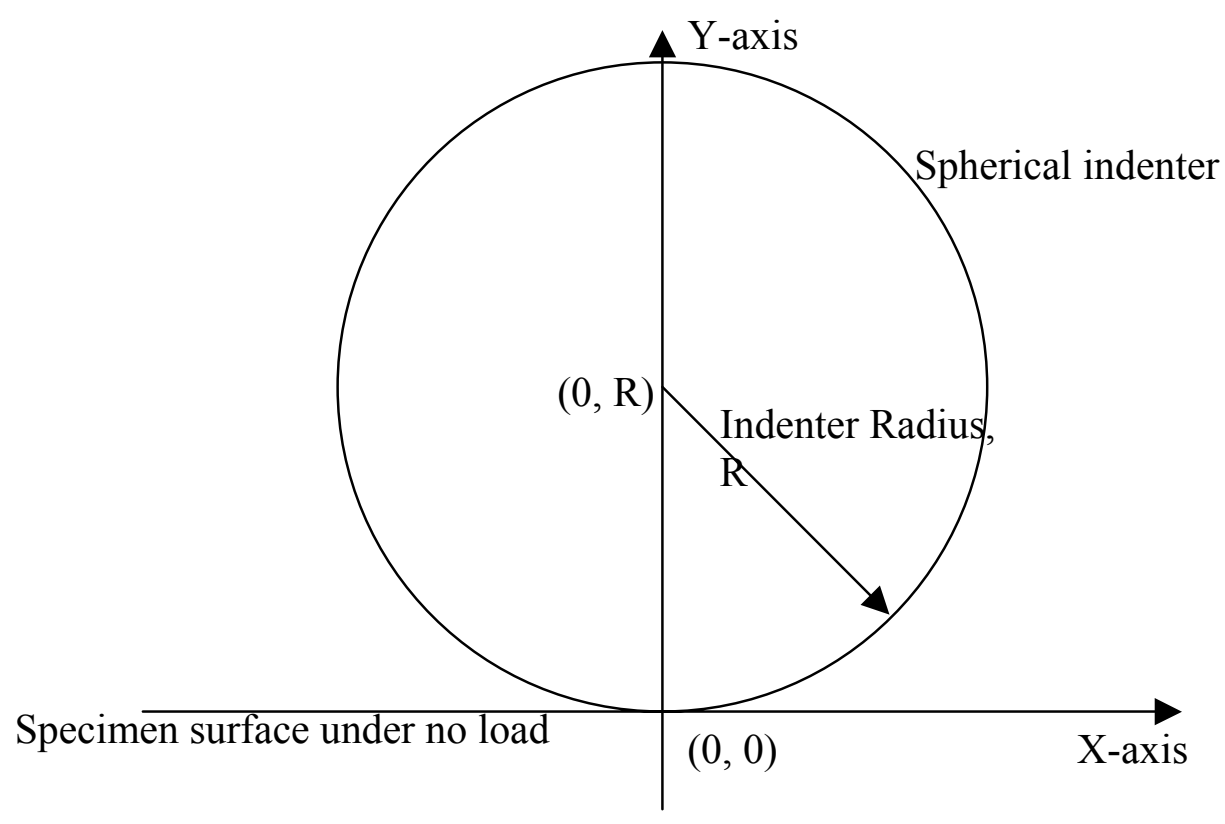

Figure 4.3 Coordinate system and the non-deformable spherical indenter, the specimen surface under no load is the same as the $\mathrm{x}$-axis.

The assumption that the indenter is a spherical rigid body is fundamental during the development of the procedure. Figures 4.4 and 4.5, shows a spherical indenter interacting with the specimen surface, and the Newton' rings radius. These figures are used to derive the mathematical equations that define the position of the specimen surface from the Newton's ring radius. The simplified TIM instrument provides the image in which the Newton's rings radii are measured, and then related to the geometry of the indenter, as shown in Figures 4.4 and 4.5.

The geometrical relation between the reflected white light from the specimen surface and the generated Newton's ring radius are shown in Figure 4.4. 
Sapphire half ball, diameter $1.5 \mathrm{~mm}$

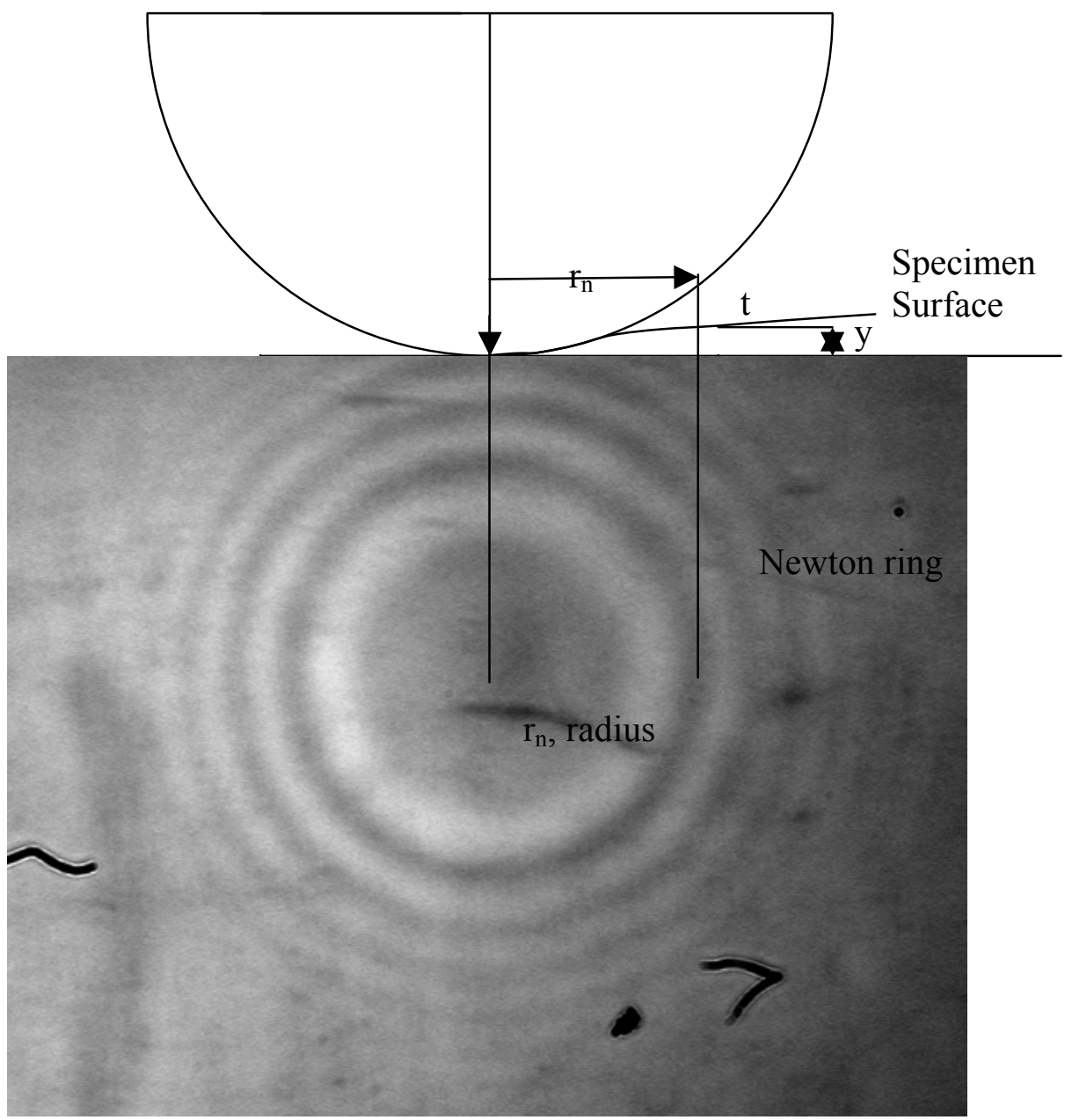

Figure 4. 4 Spherical indenter, specimen surface and Newton ring radius. 
Figure 4.5 shows the geometrical relations that were developed to calculate the position of the specimen's surface. The quantities involved are: the radius of the spherical nondeformable indenter $(\mathrm{R})$, the experimentally measured Newton's ring radius $\left(\mathrm{r}_{\mathrm{n}}\right)$, and the calculated air thickness film (t) (2.56) necessary to the path interference that produces the dark and bright rings.

Half ball sapphire tip indenter

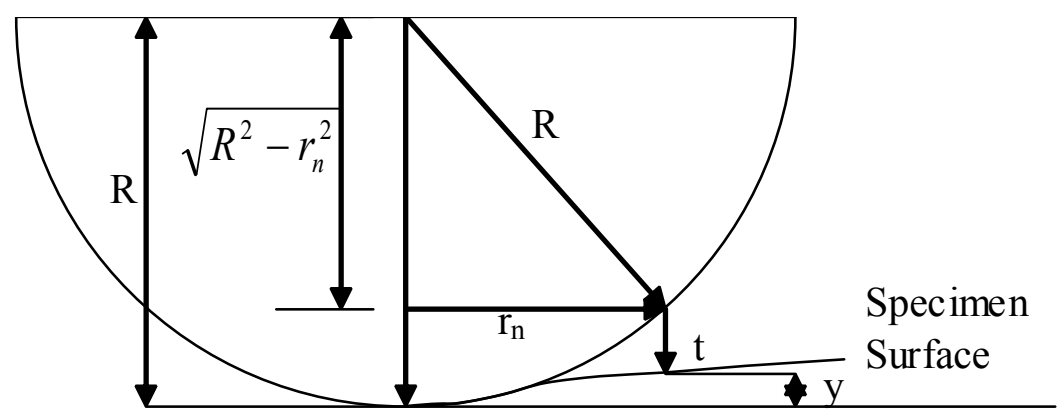

Figure 4. 5 Geometrical relations to determine the specimen surface position from the Newton's rings radius.

An image produced by the TIM instrument and its associated graph is illustrated on Figure 4.6. 


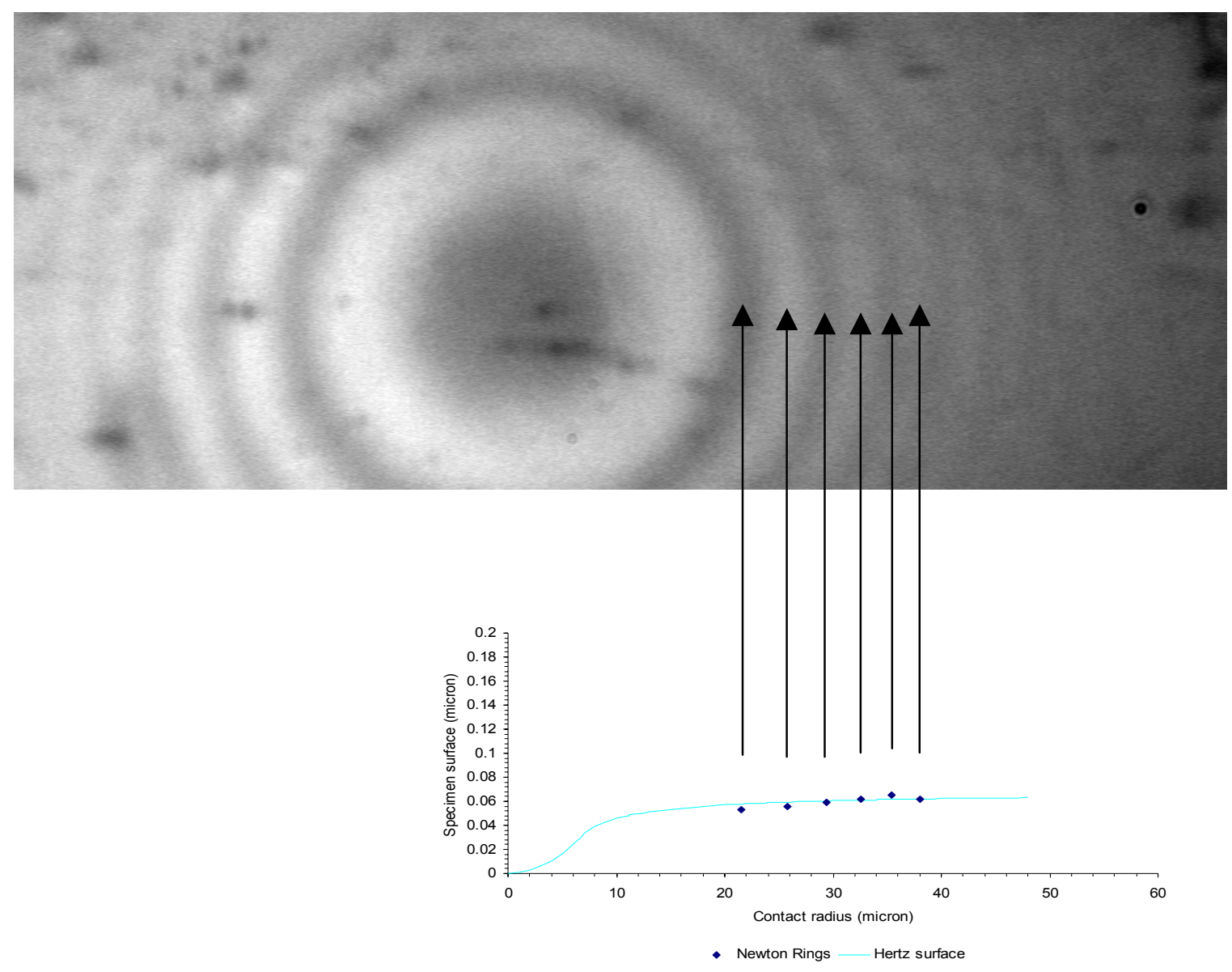

Figure 4.6 Simplified TIM's image and the associated graph after the present procedure were applied.

The following equations defines the point where the light is reflected from the specimen surface to generate the $\left(r_{n}\right)$ Newton ring as derived from the geometrical relations shown in Figure 4.5:

$y=R-\left(t+\sqrt{R^{2}-r_{n}^{2}}\right)$

where

$\mathrm{R}=$ Indenter radius

$\mathrm{t}=$ Air gap

$\mathrm{r}_{\mathrm{n}}=$ Newton's Ring radius. 
Equation (4.1) determines the position of the reflection point over the specimen surface. The point on the specimen surface must be in agreement with the deformed specimen surface predicted by Hertz's equation. Hertz equation for a specimen surface outside the contact area is [7]:

$$
u_{z}=\left(\frac{1-v^{\prime 2}}{E^{\prime}}+\frac{1-v^{2}}{E}\right) \frac{3}{2} p_{m} \frac{1}{2 a}\left[\left(2 a^{2}-r^{2}\right) \sin ^{-1} \frac{a}{r}+r^{2} \frac{a}{r}\left(1-\frac{a^{2}}{r^{2}}\right)^{1 / 2}\right] r \geq a
$$

In the following discussion the (r) distance position will be substituted by the n-Newton rings radius $\left(\mathrm{r}_{\mathrm{n}}\right)$.

The mean contact pressure is defined by:

$$
\mathrm{p}_{\mathrm{m}}=\frac{\mathrm{P}}{\pi \mathrm{a}^{2}}
$$

The contact radius is defined by the following equation [1]

$$
\begin{aligned}
& a^{3}=\frac{4 k P R}{3 E} \\
& \mathrm{k}=\left(\frac{9}{16}\right)\left[\left(1-v^{2}\right)+\left(1-v^{\prime 2}\right) \frac{\mathrm{E}}{\mathrm{E}^{\prime}}\right]
\end{aligned}
$$

If Equations (4.5) and (4.4) are combined, the elastic modulus of the material is a function of the contact radius, load, indenter radius, and the material properties of the indenter. The equation is given by:

$$
E=\frac{1-v^{2}}{\left[\frac{4}{3 R} \frac{a^{3}}{P}-\frac{\left(1-v^{\prime 2}\right)}{E^{\prime}}\right]}
$$

Equations (4.2) and (4.6) can be combined to obtain de surface deformation as a function of the contact radius (a), then, equation (4.7) is obtained. 
$u_{z}=\frac{1}{\pi R}\left[\left(2 a^{2}-r^{2}\right) \sin ^{-1} \frac{a}{r}+r^{2} \frac{a}{r}\left(1-\frac{a^{2}}{r^{2}}\right)^{1 / 2}\right] r \geq a$

where

$\mathrm{r}=$ Distance from the center of indentation

Equation (4.7) is a function of the indenter radius (R), Newton's ring radius $\left(r=r_{n}\right)$, and the unknown contact area (a). Equation (4.7) does not involve the elastic modulus and Poisson's ratio of the material and indenter. That means the proposed method does not require a previous knowledge of the elastic modulus and Poisson's ratio of the material under test.

The origin of the coordinate system for Equation (4.7) is set at the maximum depth of penetration (h) [7].

$$
h=\frac{a^{2}}{R}
$$

and

$$
y=h-u_{z}
$$

Substituting Equations (4.7) and (4.8) into Equation (4.9), the equation to find the contact radius becomes:

$$
y=\frac{a^{2}}{R}-\frac{1}{\pi R}\left[\left(2 a^{2}-r^{2}\right) \sin ^{-1} \frac{a}{r}+r^{2} \frac{a}{r}\left(1-\frac{a^{2}}{r^{2}}\right)^{1 / 2}\right] r \geq a
$$

To find the contact radius, Equation (4.10) is used with the following numerical optimization.

$$
\begin{aligned}
& f(x)=h-u_{z}-y=0 \\
& f(x)=0=\frac{a^{2}}{R}-\frac{1}{\pi R}\left[\left(2 a^{2}-r^{2}\right) \sin ^{-1} \frac{a}{r}+r^{2} \frac{a}{r}\left(1-\frac{a^{2}}{r^{2}}\right)^{1 / 2}\right]-y \quad r \geq a
\end{aligned}
$$


Using the experimental Newton's ring radii, Equation (4.1) and (4.10), the position of the specimen surface is then calculated. Table 4.1 summarizes an example using this procedure.

Table 4.1 Contact radius evaluation

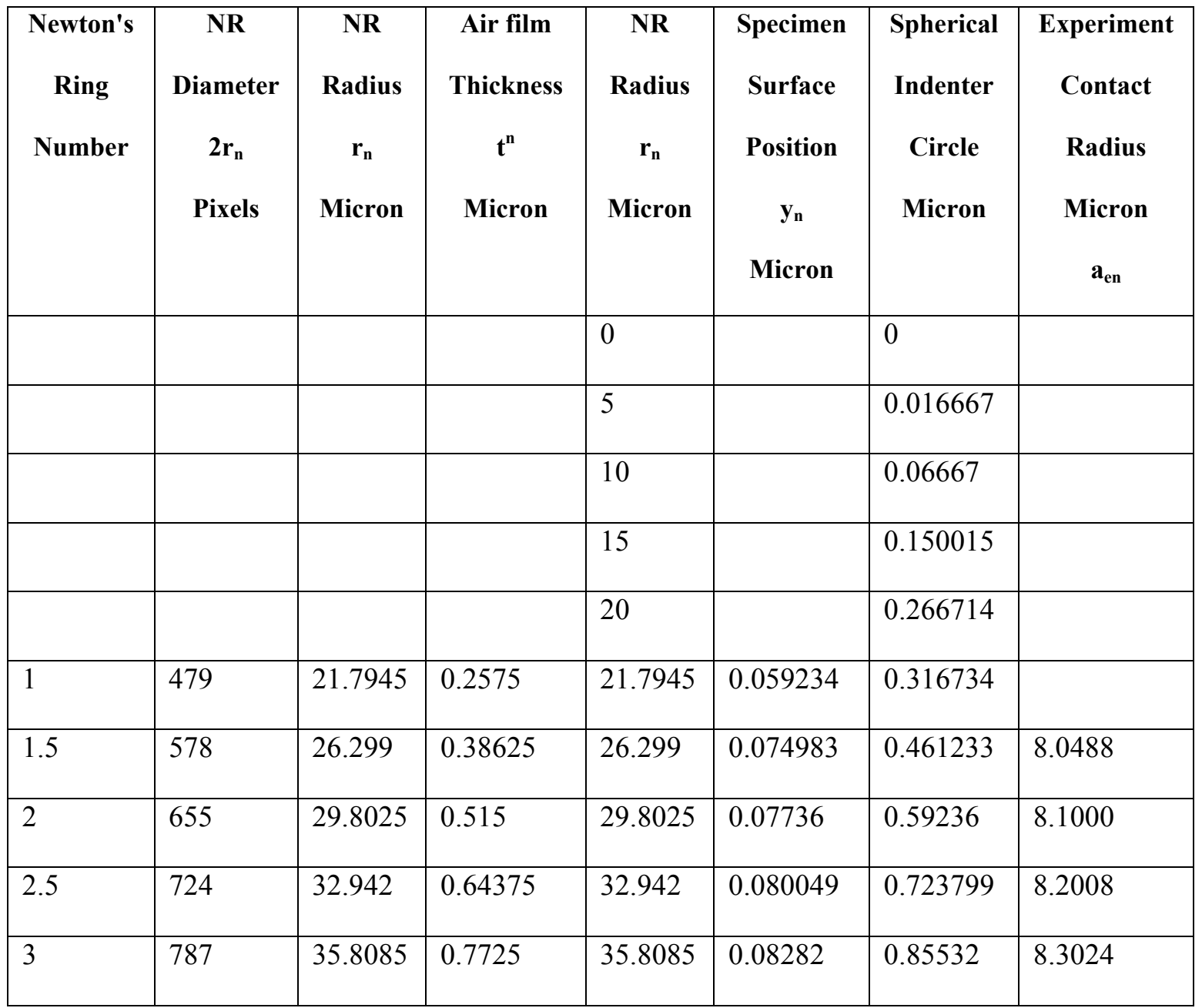

The values of light wave length and factor to conversion pixels to microns on the picture used on this research are the following:

Light wave length $=0.515$ microns

$0.091 * \mathrm{n}($ pixels $)=$ microns

Indenter radius $=750$ microns 
When the reflection points are determined, the points are substituted into Equation (4.12), and the contact radius is obtained. The average value of the available Newton's rings radius is the value used in Equation (4.6) to calculate the elastic modulus of the material under test.

The following table summarizes the mechanical properties of the materials tested and the properties of the indenter:

Table 4. 2 Mechanical properties of the tested materials

\begin{tabular}{|l|c|c|c|c|c|c|c|}
\hline Indenter & Materials & $\begin{array}{c}\text { Elastic } \\
\text { Modulus } \\
\text { Gpa }\end{array}$ & $\begin{array}{c}\text { Yield } \\
\text { Strength } \\
\text { MPa }\end{array}$ & $\begin{array}{c}\text { Poisson's } \\
\text { ratio }\end{array}$ & $\begin{array}{c}\text { Ratio } \\
\text { E/Y }\end{array}$ & $\begin{array}{c}\text { Reference } \\
\text { E }\end{array}$ & $\begin{array}{c}\text { Reference } \\
\text { Y }\end{array}$ \\
\hline Sapphire & 345 & & 0.29 & & {$[33]$} & \\
\hline & $\begin{array}{c}\text { Aluminum } \\
7075-T 6\end{array}$ & 71.7 & 462 & 0.33 & 155.19 & {$[34]$} & {$[34]$} \\
\hline $\begin{array}{c}\text { Inconel alloy } \\
783\end{array}$ & 177.3 & 779 & .31 & 227.599 & {$[35]$} & {$[35]$} \\
\hline & $\begin{array}{c}\text { Molybdenum } \\
\text { Alloy 695 }\end{array}$ & 243 & & & & {$[36]$} & \\
\hline
\end{tabular}

The proposed method has been applied to Aluminum Alloy 7075-T6, Inconel Alloy 783, and Molybdenum Alloy 695.The results of the surface specimen under load position determination are shown in Figures 4.7, 4.10 and 4.13. The $\mathrm{x}$-axis of represents the original specimen surface with no load while the y-axis represents the position of the point on the specimen surface where the reflection of the light occurs. The y-axis also represents the depth of indentation. Each point denotes the position where the reflection occurs for a respective Newton ring radius. The spherical indenter and the Hertz' specimen surface are shown, to reference the reflection points to the physical system.

Figures 4.8, 4.11 and 4.14 show the comparison between the experimentally determined contact radius and the Hertz equation for the studied metals. An interesting observation is that good agreement is observed not only in the elastic range, also in the early elastic-plastic stage. 
The point where the metal reaches the yield is a not well-defined value. This condition has been observed by other researchers $[14,18]$. Tabor $[12]$ proposes that the material yield start at $\mathrm{p}_{\mathrm{m}}=$ $1.1 \mathrm{Y}$, but recently, finite elements models and experimental results showed the yielding starts at $\mathrm{p}_{\mathrm{m}}=1.6 \mathrm{Y}[14,18]$. The experimental results of this dissertation agree with the later.

Figures $4.9,4.12$ and 4.15 show the calculation results for the elastic modulus. These results are in good agreement with the literature values. Figures 4.14 and 4.15 are from Molybdenum alloy 695. A deviation is observed on the behavior of Molybdenum Alloy 695 from the expected theoretical values.

The discrepancy can be attributed to the fact that the indenter and the specimen have similar elastic modulus. If the indenter is a sphere and the specimen a flat surface, the contact radius is twice that of the radius of the indenter [7]. In the present situation, Molybdenum has $324 \mathrm{GPa}$ and sapphire $345 \mathrm{GPa}$, which means that the contact radius is greater than predicted by Hertz' equation, as it was found in the experimental data.

The grain size has influence in the discrepancy between experimental and published values. Molybdenum alloy 695 has grain size grater than 25 microns, while the contact diameter is less than this value.

Tabor's analysis of the deformation of the indenter suggests that the indenter should be at least two and a half times as hard as the metal under evaluation [12]. The term "hard" is related to the Brinell hardness number or yield pressure. 


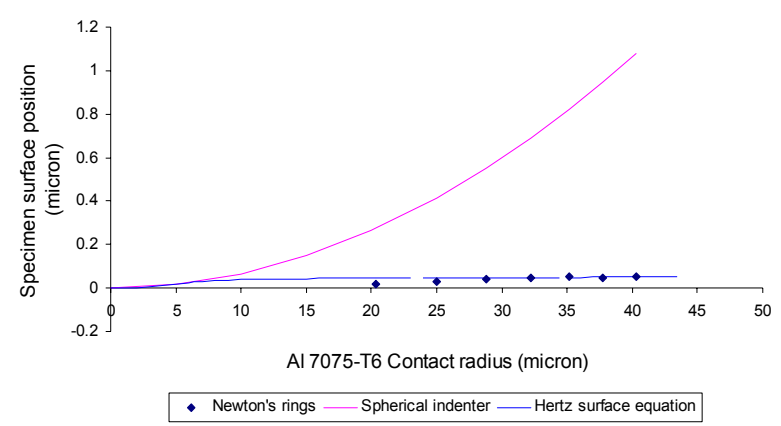

(a) Force $0.03 \mathrm{~N}$

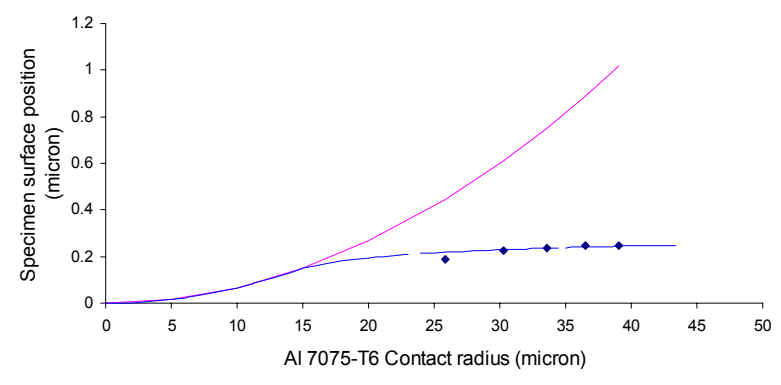

(c) Force $0.381 \mathrm{~N}$

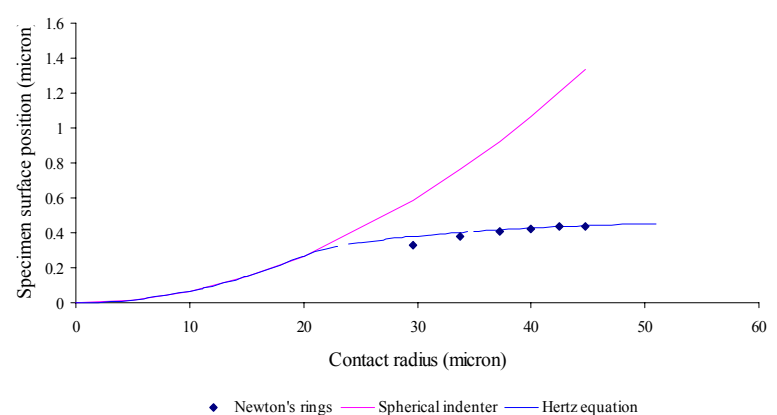

(e) Force $0.98 \mathrm{~N}$

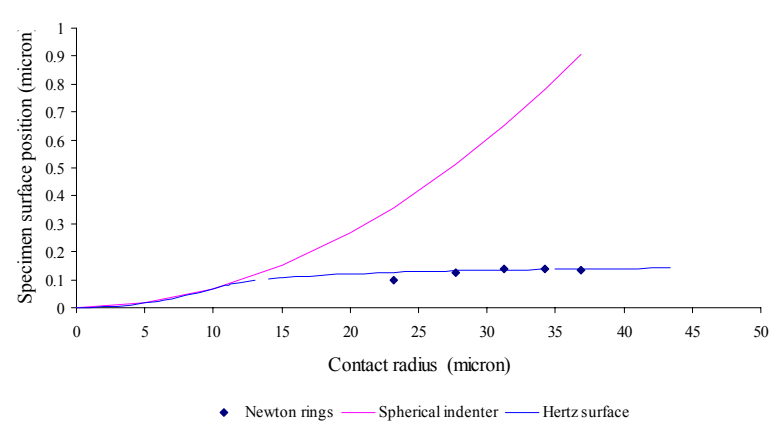

(b) Force $0.153 \mathrm{~N}$

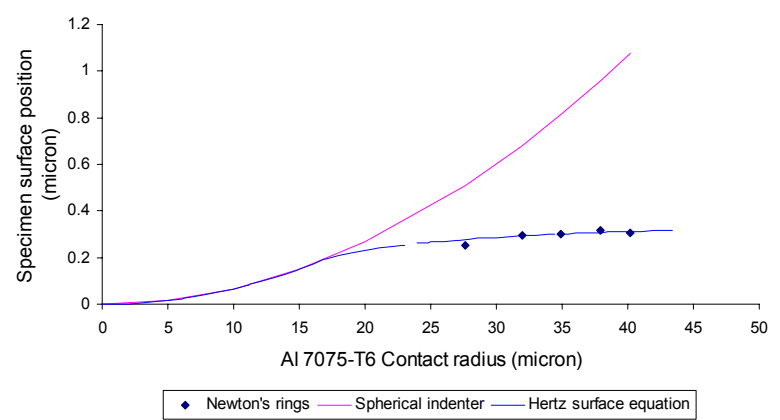

(d) Force $0.546 \mathrm{~N}$

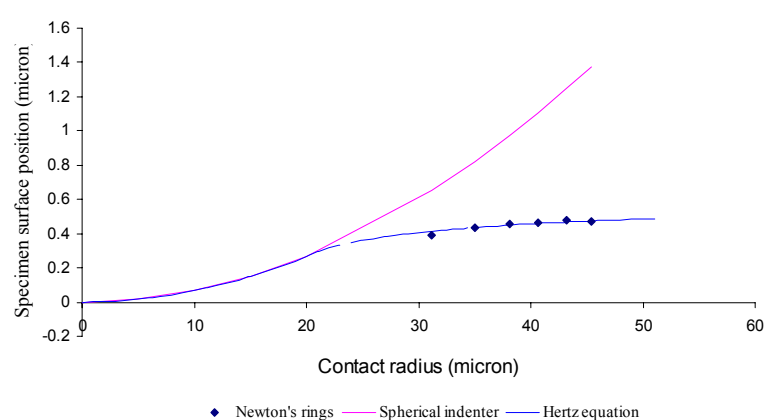

(f) Force $1.105 \mathrm{~N}$

Figure 4.7 Aluminum alloy 7075-T6. Graphs (a) to (f) show the position of the specimen surface obtained by the Newton's ring method under different loads. The Hertz surface is superimposed to compare with the experimental data. 


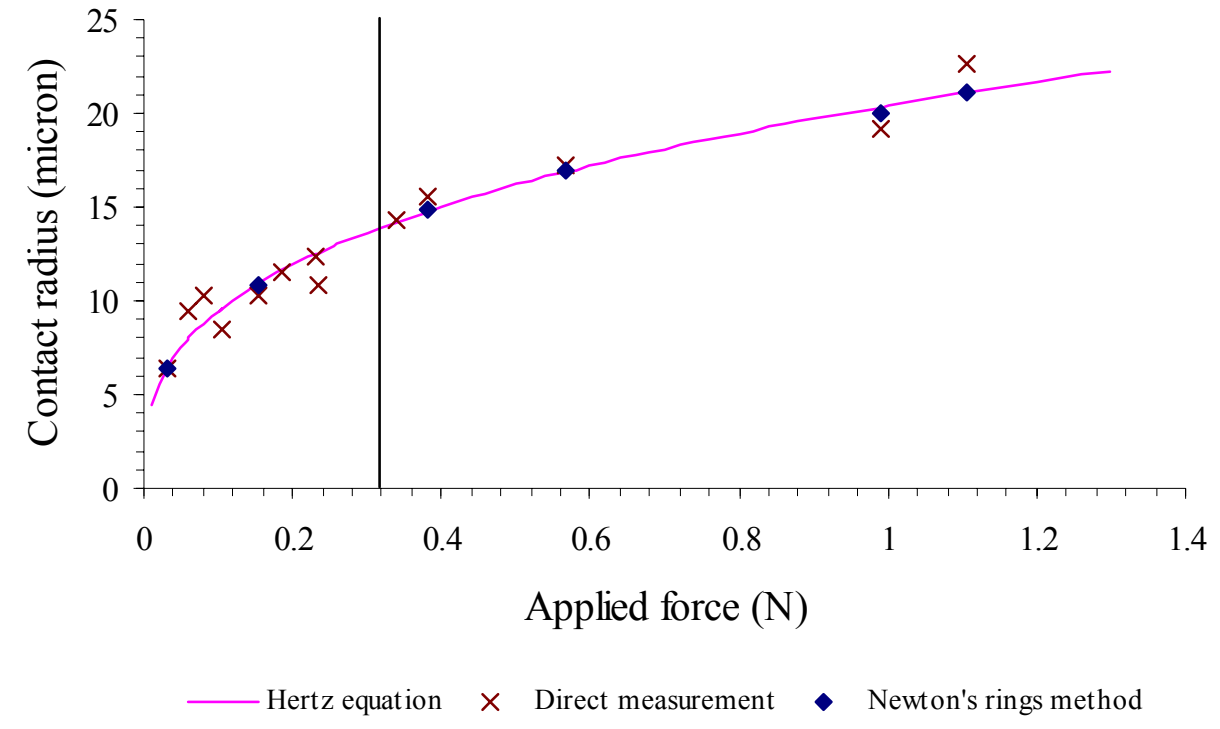

Figure 4.8 Contact radius obtained by the Newton's rings method in agreement with the Hertz equation for Aluminum alloy 7075-T6

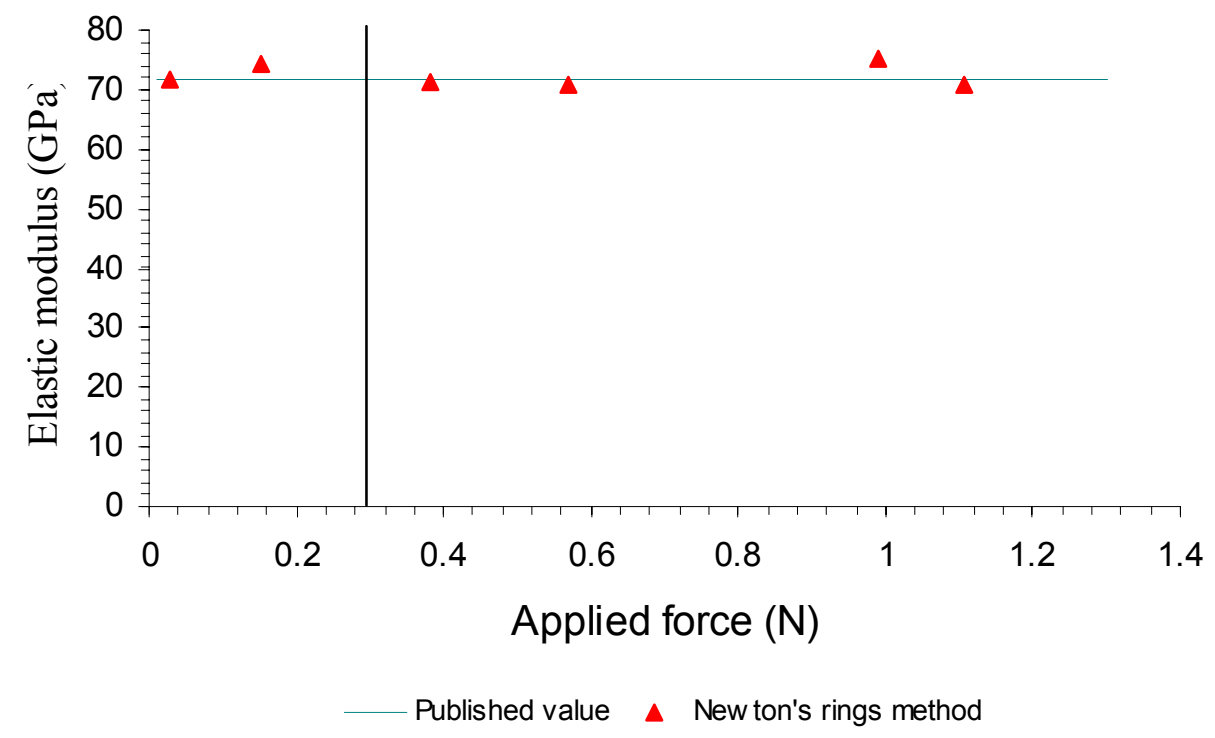

Figure 4. 9 Experimental elastic modulus for Aluminum 7075-T6 obtained by the Newton's ring method. 


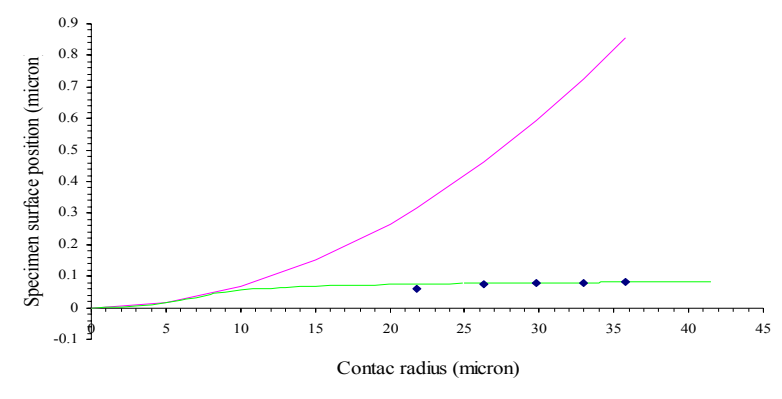

(a) Force $0.127 \mathrm{~N}$

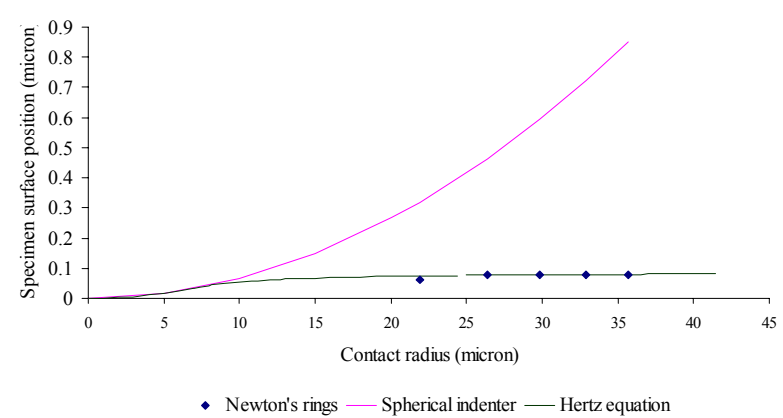

(c) Force $0.125 \mathrm{~N}$

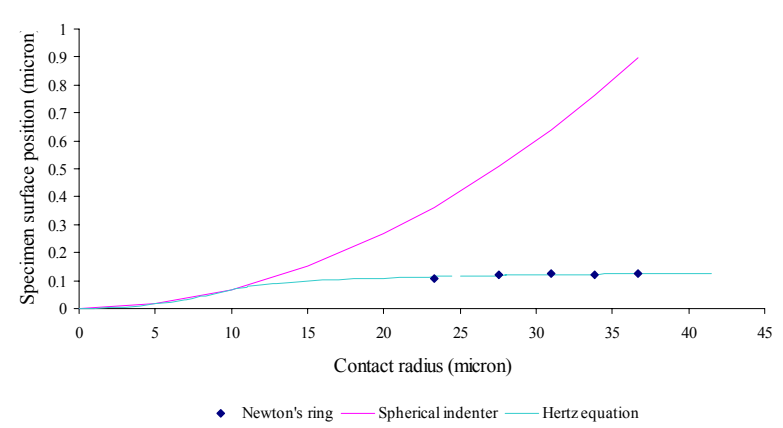

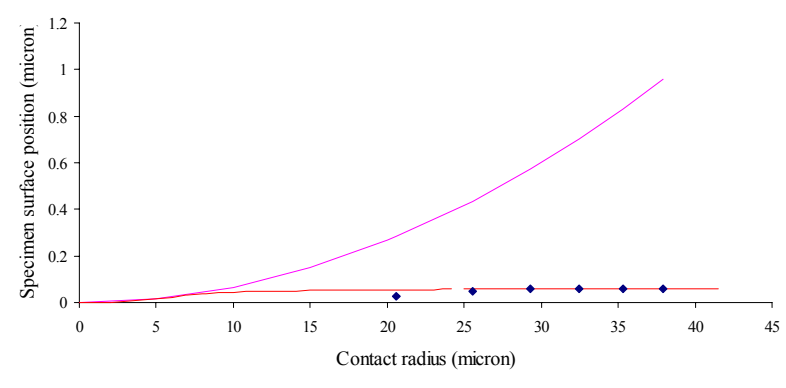

(b) Force $0.077 \mathrm{~N}$

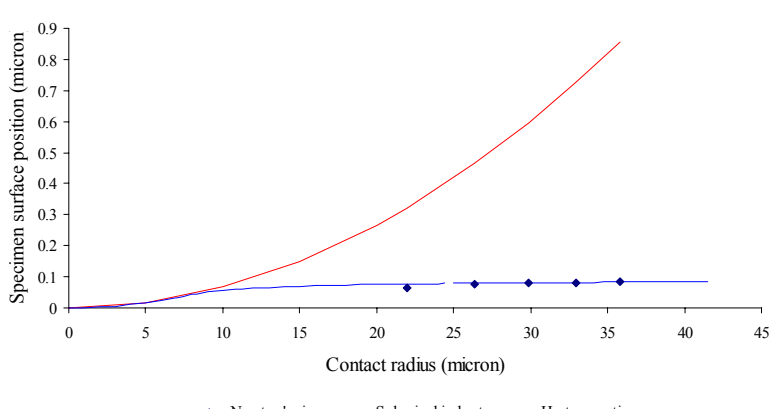

(d) Force $0.131 \mathrm{~N}$

(e) Force $0.249 \mathrm{~N}$

Figure 4.10 Inconel 783 Graphs (a) to (e) show the position of the specimen surface obtained by the Newton's ring method under different loads. The Hertz surface is superimposed for comparison with the experimental data. 


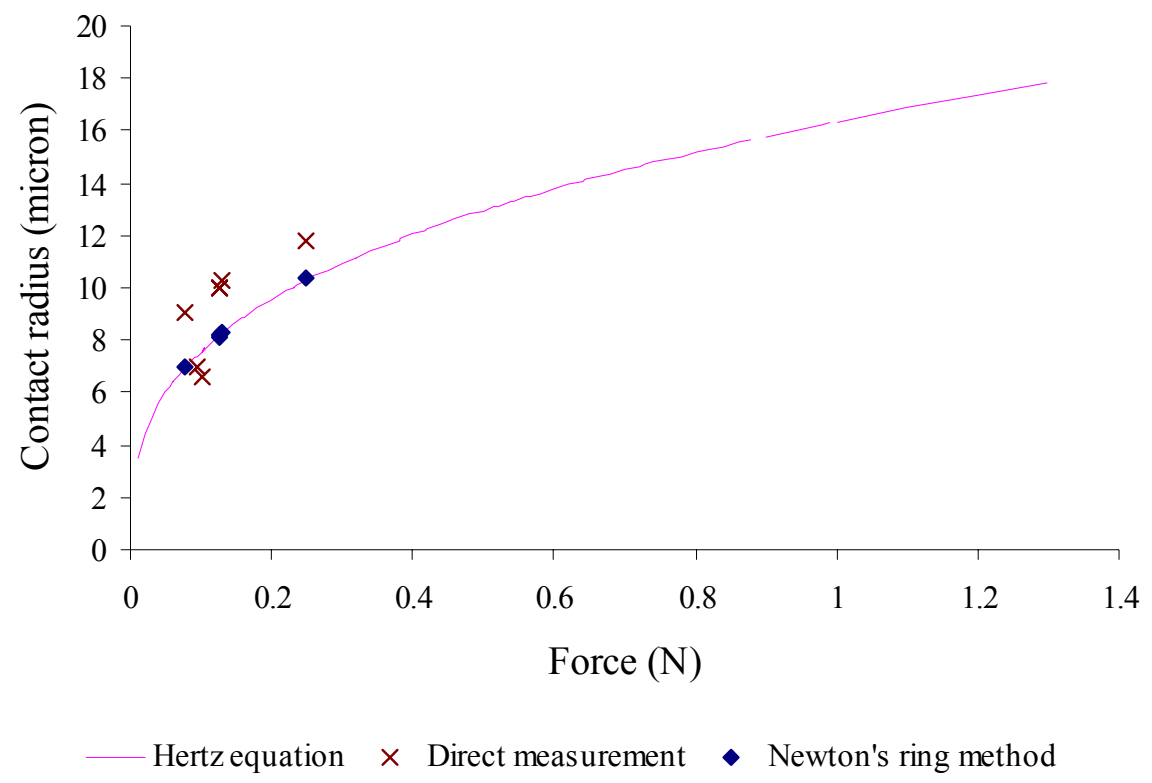

Figure 4.11 Contact radius obtained by the Newton's rings method in agreement with the Hertz equation for Inconel 783.

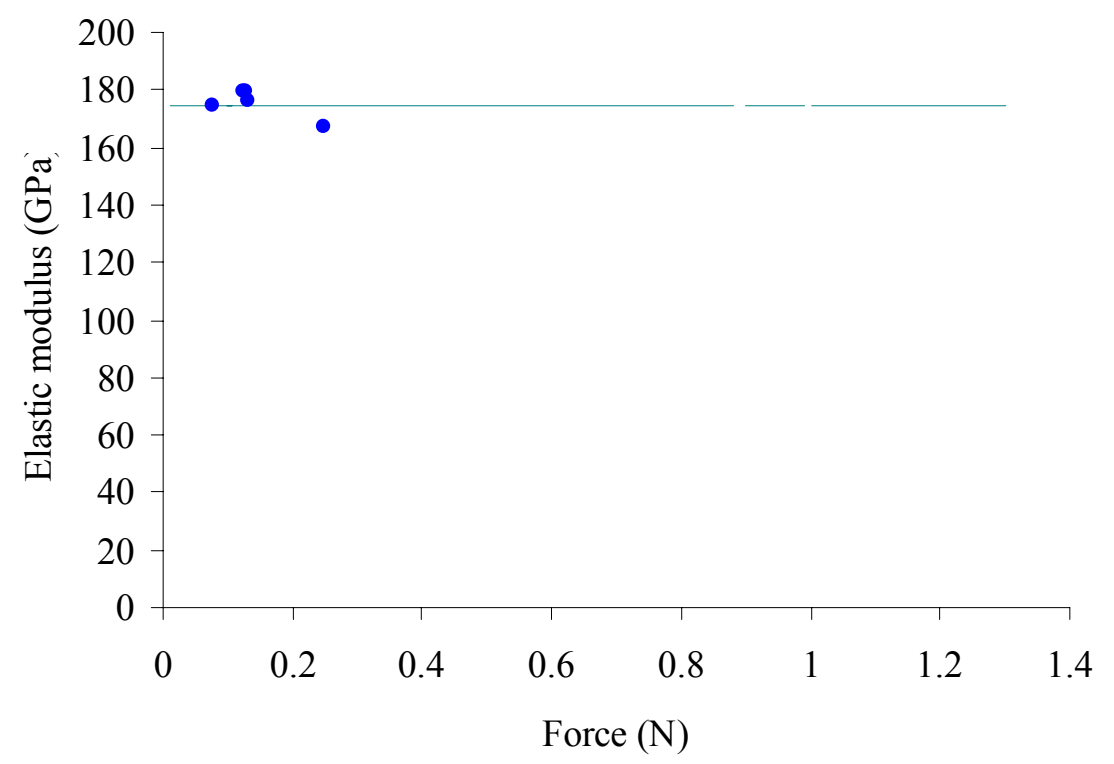

Figure 4.12 Experimental elastic modulus for Inconel 783 obtained by the Newton's rings to determine the specimen surface position. 


\section{MOLYBDENUM ALLOY 695}

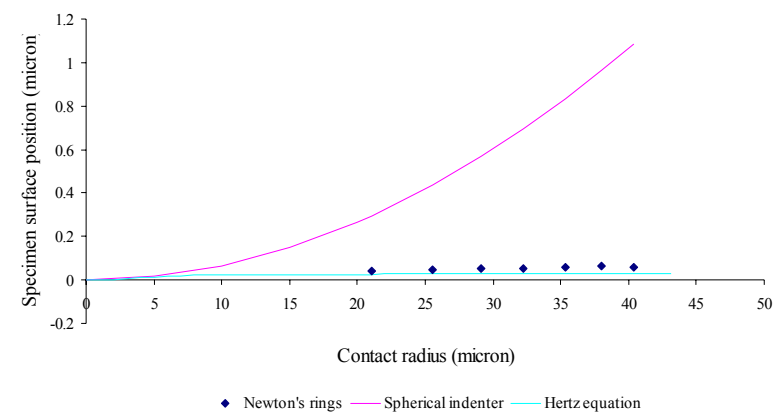

(a) Force $0.03 \mathrm{~N}$

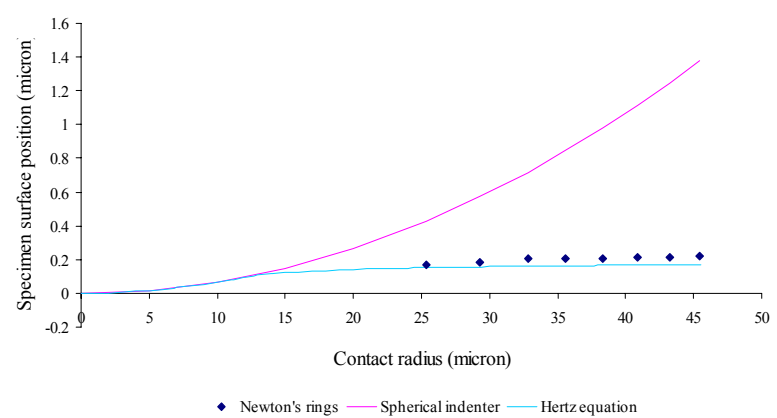

(c) Force $0.479 \mathrm{~N}$

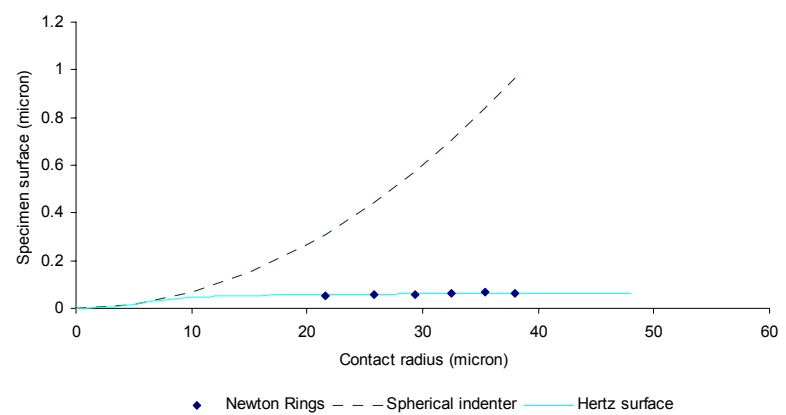

(b) Force $0.1 \mathrm{~N}$

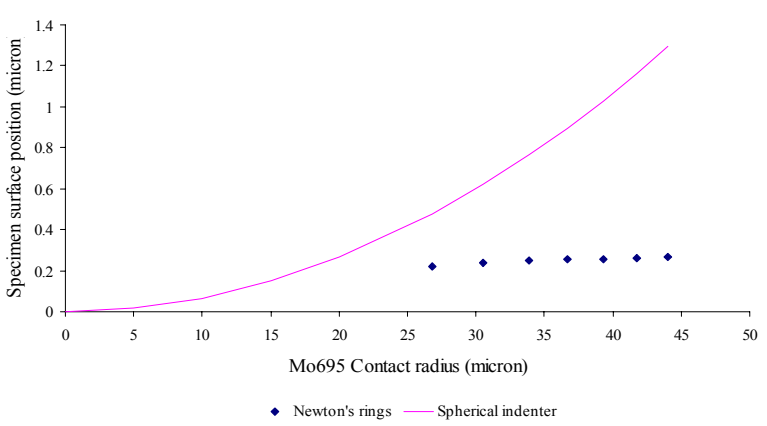

(d) Force $0.649 \mathrm{~N}$

Figure 4. 13 Molybdenum Alloy 695. Graphs (a) to (e) show the position of the specimen surface obtained by the Newton's ring method under different loads. The Hertz surface is superimposed to compare with the experimental method. 


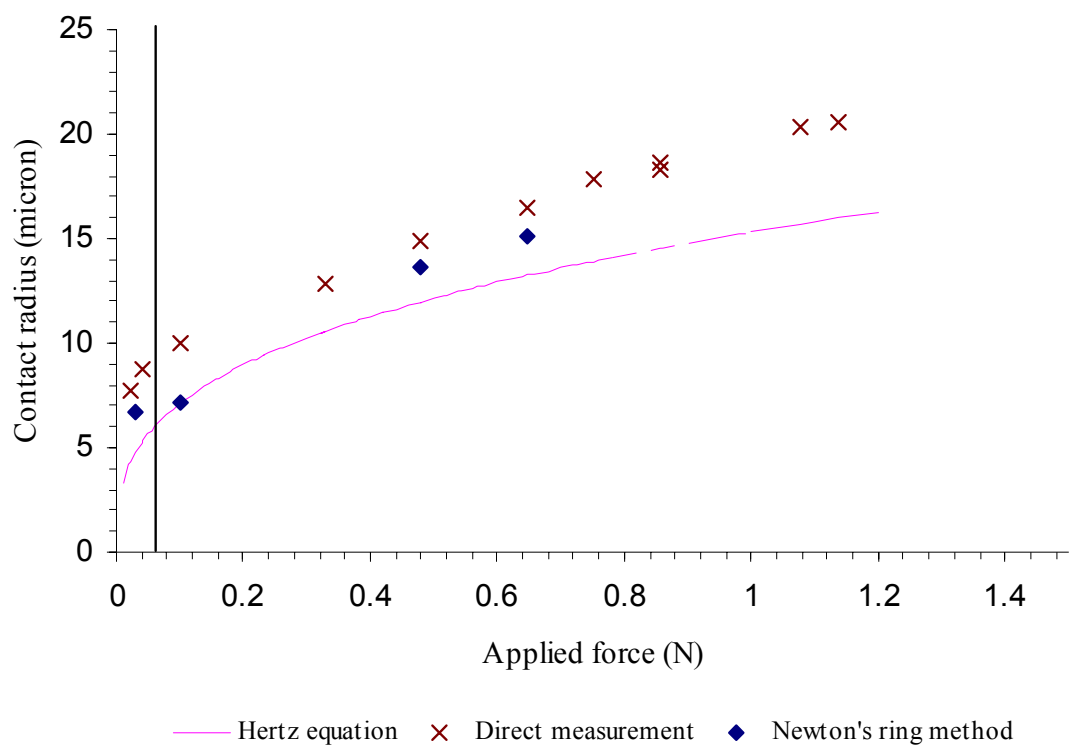

Figure 4.14 Contact radius obtained by the Newton's ring method in agreement with the Hertz equation for Molybdenum alloy 695.

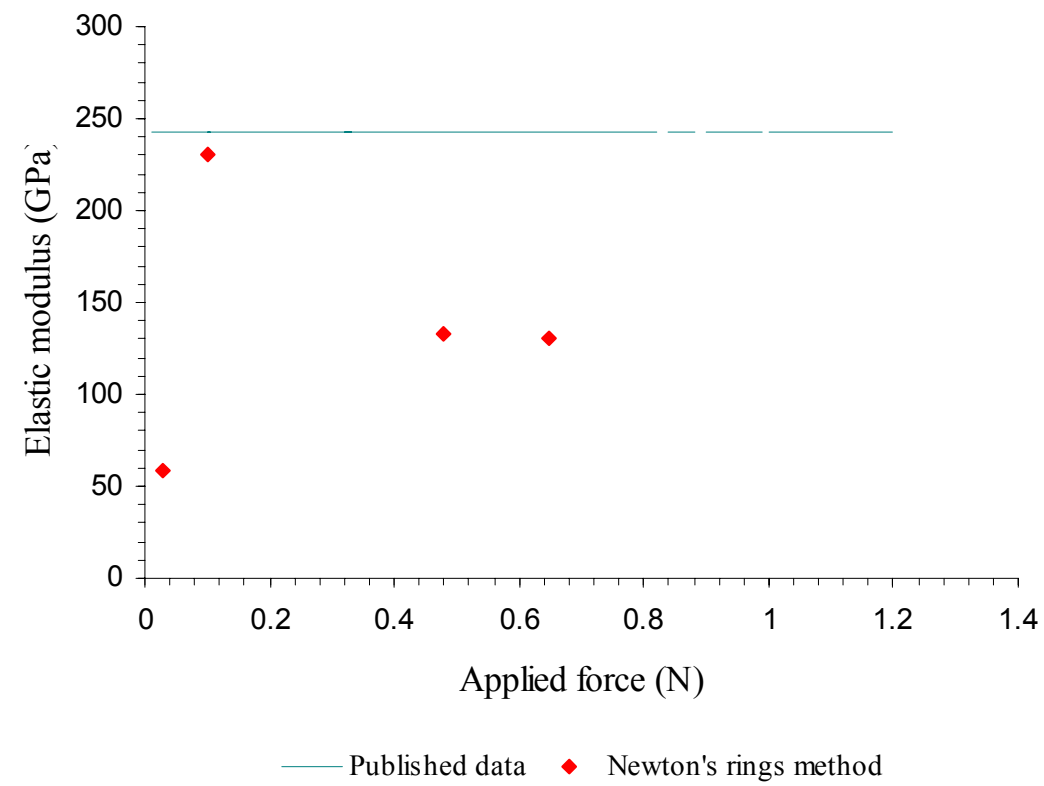

Figure 4.15 Experimental elastic modulus for Molybdenum Alloy 695 obtained by the Newton'srings method. 


\subsubsection{Oliver and Pharr model}

The application of instrumented indentation testing to determine the elastic modulus by nanoindenters is now a standardized process [22]. In this research we follow the operational sequence outlined in the ASTM draft standard for the SSM and ABI test [19], due to some similarities between the simplified TIM instrument and the SSM. The testing process to obtain the elastic modulus by the TIM instrument is described in the following sections.

\subsubsection{Initial Preload:}

When the tip indenter touches the surface, there is an initial penetration by the tip indenter. Independent of how small the magnitude of the load is, it is necessary to calculate the penetration produced by this initial load in order to correct the raw load piezoelectric displacement data. All the existent nano or micro indentation instruments in the market require the determination of a preload initial indentation point. For example, the ABI method utilizes forces of $2 \mathrm{~N}, 5 \mathrm{~N}$, and $10 \mathrm{~N}$ for ball diameters between $0.254 \mathrm{~mm}$ to $0.762 \mathrm{~mm}$ [17]. The simplified TIM instrument uses the Newton ring as a means to detect the contact surface of the specimen. Then, the indenter is instructed to begin the indentation test. The Newton ring can facilitate control the indentation test, to ensure no plastic damage is produced in the specimen. The magnitude of the applied stresses is a function of the indenter radius. A few $\mathrm{mN}$ of force are enough to reach the material yield point using a sphere of $1.5 \mathrm{~mm}$ of diameter. Using the TIM instrument and aided by the Newton rings, we can conduct initial alignment without damage on the material surface or residual impression.

The materials that provide good resolution of indentation in the elastic range are materials with low E/ $\sigma_{\mathrm{y}}$ ratio. For example, $7075-\mathrm{T} 6$ aluminum provides low Young's modulus and high yield strength values, necessary for a low value $\mathrm{E} / \sigma_{\mathrm{y}}$ ratio. 


\subsubsection{Single Cycle of Loading and Unloading}

To begin the indentation process, the indenter is positioned near the specimen surface and a command is sent to the piezoelectric control via the computer software. This starts the actuator motion. The piezoelectric begins the indentation with a periodic pre-programmed loading and unloading. At the final stage of the cycle, unloading sequence, when no load is sensed by the load cell, a command is sent to the piezoelectric control to stop the cycle. That means there is no contact between the indenter and the specimen surface. The image of the contact zone of indentation is taken at maximum load.

Figure 4.16 shows the raw data of piezoelectric tip displacement and the applied force. This raw data are automatically stored by a computer program to a text file and processed using Excel $^{\mathrm{TM}}$ software.

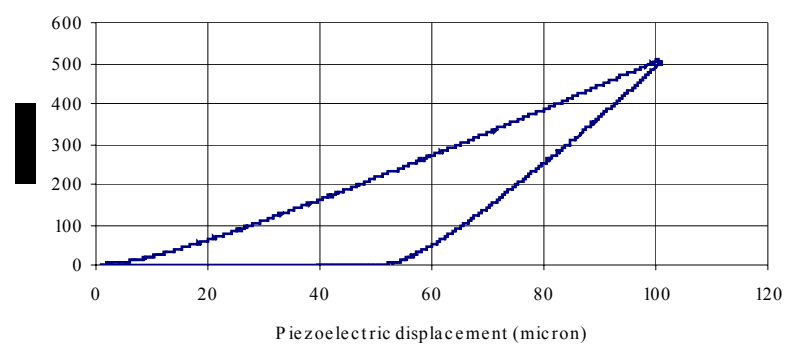

Figure 4. 16 Example of raw data from the simplified TIM instrument.

\subsubsection{Example of Initial Penetration Correction}

In Figure 4.17 the preload is associated to the $1.2 \mathrm{~N}$ of initial contact. The preload is the force applied to the material as the initial penetration occurs. To calculate the penetration distance associated with the preload, the force-piezoelectric displacement data is fitted with a linear regression. The quantity of points is arbitrary chosen, but must be in the elastic range. The 
intersection between the curve fitting and the displacement axis provides the initial displacement, obtained by extrapolation. The calculated initial penetration distance is added to the raw loadpiezoelectric displacement data. Figure 4.17 shows an example of the data taken for the linear interpolation of the initial penetration data.

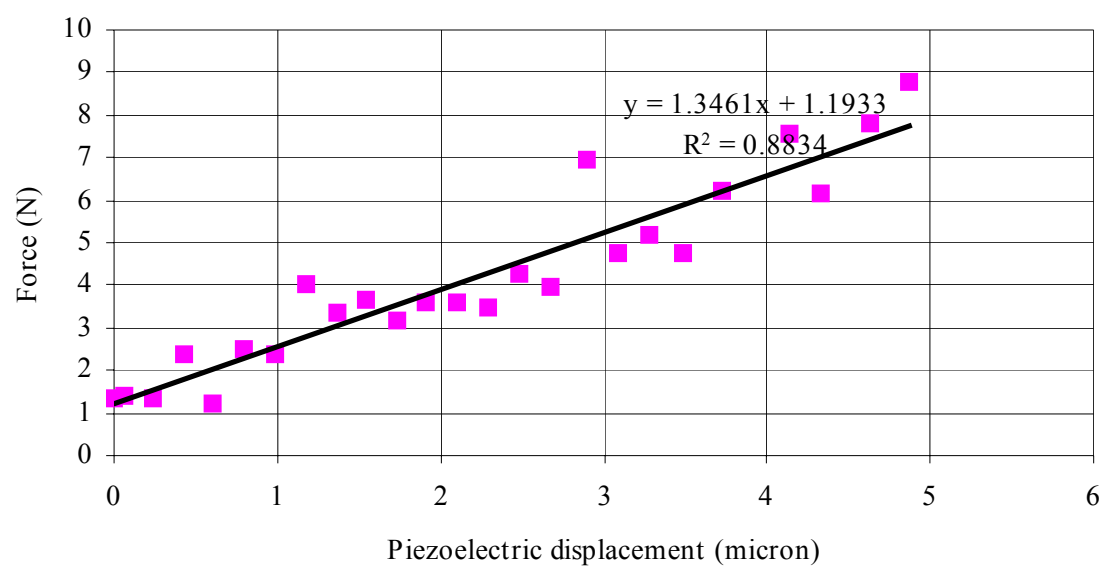

Figure 4.17 An example of the calculation of the initial penetration correction due to the initial contact preload of $1.2 \mathrm{~N}$.

\subsubsection{Correction by instrument compliance}

The raw data obtained from the load-piezoelectric displacement curve, corrected by initial preload, contains the deformation of the specimen and the deformation of all parts of the simplified TIM instrument i.e., frame, piezoelectric actuator, specimen mount, load cell, and the piezoelectric adapters. To find deformations other than that of the specimen, it is assumed that the total deformation is the sum of two springs connected in series [13], given by:

$\mathrm{h}_{\mathrm{t}}=\mathrm{h}_{\mathrm{f}}+\mathrm{h}_{\mathrm{s}}$

where

$\mathrm{h}_{\mathrm{t}}=$ Total deformation 


$$
\begin{aligned}
& h_{f}=\text { Frame deformation } \\
& h_{s}=\text { Specimen deformation }
\end{aligned}
$$

Assuming linear response and solving for the displacement, the force is given by:

$$
\begin{aligned}
& \mathrm{P}=\mathrm{kh} \\
& \mathrm{h}=\mathrm{P} / \mathrm{k} \\
& \text { where } \\
& \mathrm{h}=\text { Displacement } \\
& \mathrm{P}=\text { Force } \\
& \mathrm{k}=\text { Stiffness }
\end{aligned}
$$

For springs connected in series:

$$
\begin{aligned}
& \frac{P}{k_{t}}=\frac{P}{k_{f}}+\frac{P}{k_{s}} \\
& \frac{1}{k_{t}}=\frac{1}{k_{f}}+\frac{1}{k_{s}}
\end{aligned}
$$

Using the inverse of the stiffness, the compliance is:

$$
\mathrm{C}_{\mathrm{t}}=\mathrm{C}_{\mathrm{f}}+\mathrm{C}_{\mathrm{s}}
$$

where

$$
\begin{aligned}
& \mathrm{C}_{\mathrm{t}}=\text { Total compliance } \\
& \mathrm{C}_{\mathrm{f}}=\text { Frame compliance } \\
& \mathrm{C}_{\mathrm{s}}=\text { Sample compliance }
\end{aligned}
$$


Oliver and Pharr [13] demonstrated that for all axis-symmetric indenters there is a constant relationship between the elastic contact stiffness $\mathrm{S}$, the projected area of contact, $\mathrm{A}$, and the reduced or indentation modulus $\mathrm{E}^{*}$ given by [17]:

$\mathrm{S}=\frac{\mathrm{dP}}{\mathrm{dh}}=\frac{2 \mathrm{E} * \sqrt{\mathrm{A}}}{\sqrt{\pi}}$

where

$\mathrm{S}=$ Contact stiffness

$\mathrm{dP} / \mathrm{dh}=$ Contact Stiffness

$\mathrm{E}^{*}=$ Reduced Modulus

$\mathrm{A}=$ Contac Area

The inverse of the contact stiffness, defined in Equation (4.19) is the elastic compliance of the unloading part of the graph, given by:

$\frac{1}{S}=C_{s}=\frac{\sqrt{\pi}}{2 E * \sqrt{A}}$

where

$\mathrm{C}_{\mathrm{s}}=$ System compliance

Plugging Equation (4.20), into Equation (4.18), the following equation is obtained:

$\mathrm{C}_{\mathrm{t}}=\mathrm{C}_{\mathrm{f}}+\frac{\sqrt{\pi}}{2 \mathrm{E} * \sqrt{\mathrm{A}}}$

In this work the area is calculated from the measured radius using the simplified TIM method. Note that the Oliver and Pharr method is based on the measurement of the penetration depth. By using an area function, the contact area is then calculated. The system compliance is calculated by plotting the total compliance versus the inverse of the indentation radius. The 
points on the diagram are fitted by a linear regression. The intercept of the curve fitting with the axis of the compliance is the compliance of the frame. This equation is given by:

$$
\mathrm{C}_{\mathrm{t}}=\left(\frac{1}{2 \mathrm{E}^{*}}\right)\left[\frac{1}{\mathrm{a}}\right]+\mathrm{C}_{\mathrm{f}}
$$

Figure 4.18 represents the curve fitting of the upper unloading part of Figure 4.16. The load-distance data is fitted using linear regression and the value of the slope of the resultant equation is the contact stiffness. This information will be used to calculate the system compliance.

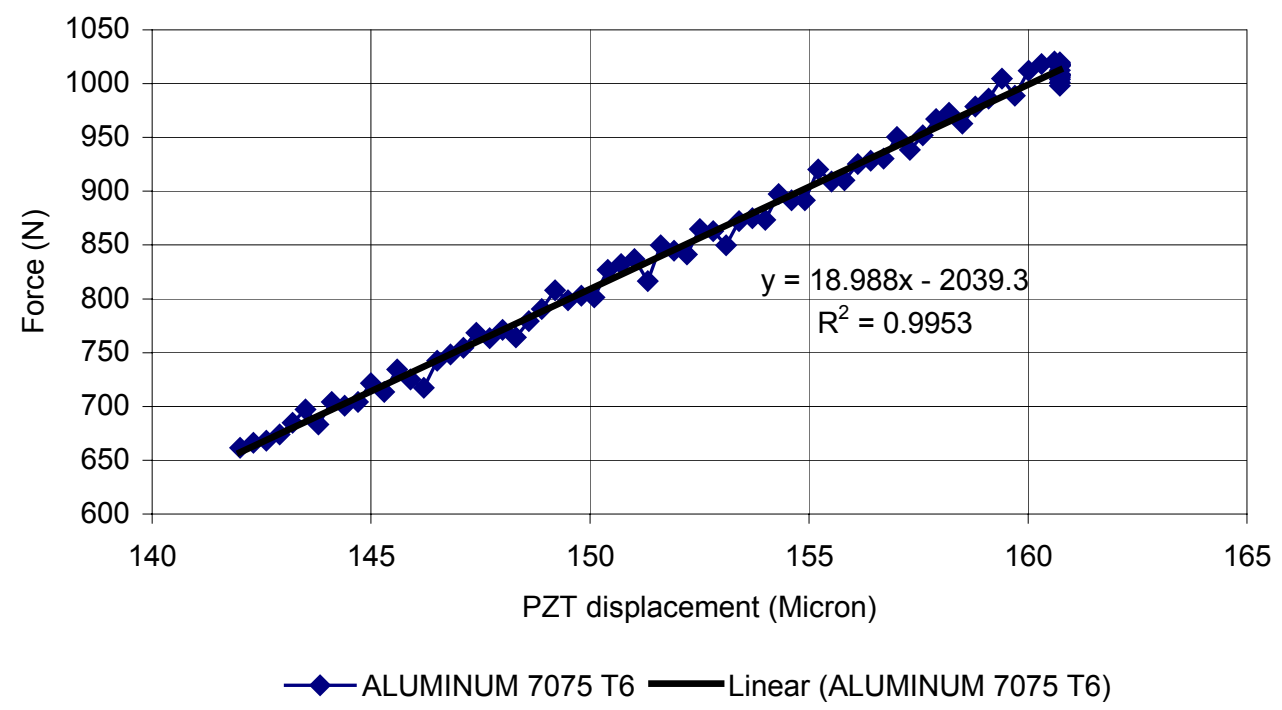

Figure 4.18 Example of the unloaded part of the graph given as PZT displacement versus load. The raw data is corrected by initial penetration.

The system compliance is calculated from Figure 4.19. The contact stiffness is obtained from a series of indentation test runs at different penetration distances. In Figure 4.19, the inverse of the contact stiffness is plotted against the inverse of the residual impression radius. In accordance with Equation (4.22) the plot should be a straight line with a slope proportional to 
$1 / 2 \mathrm{E}^{*}$ [7]. The system compliance is given by the intercept of the plot with the axis of $\mathrm{dh} / \mathrm{dP}$. Commercial software is used to fit the experimental data to a linear relation. By extrapolation, the intercept of the line with the $\mathrm{dh} / \mathrm{dP}$ axis is the frame compliance that is used to modify the raw data.

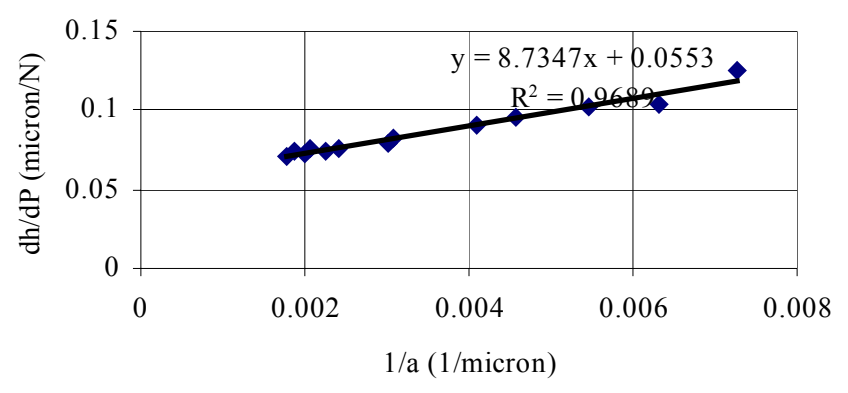

Figure 4.19 The inverse of contact stiffness is plotted against the inverse of the contact radius.

Figure 4.20 shows the load-displacement data corrected by system compliance. This graph represents the applied load and the penetration depth of the indenter into the material.

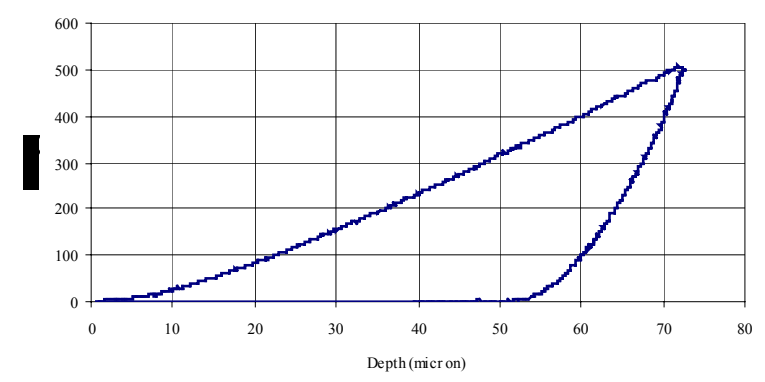

Figure 4.20 Depth-load curve for Aluminum 7075-T6. The raw data was corrected by initial penetration and system compliance. 
Figure 4.20 contains the data corrected by initial penetration and systems compliance. This graph represents the actual penetration of the indenter into the specimen material and this graph is used to calculate the contact stiffness in order to find the elastic modulus of the material.

Figure 4.21 represents the upper third of the unload part of load-unload cycle showed in Figure 4.20. From Figure 4.21 the contact stiffness is calculated and used on Equation 4.23 to calculate the reduced modulus of the material.

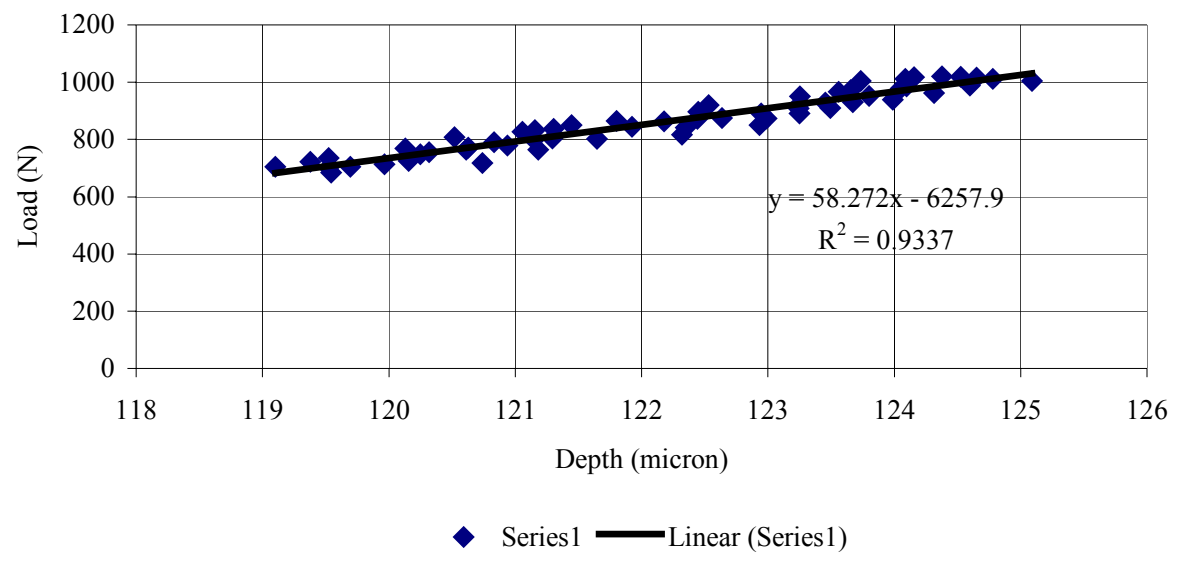

Figure 4.21 A third of the unload curve corrected by the system compliance.

Thus, the value calculated with the use of Figure 4.19 gives the machine compliance $\left(\mathrm{C}_{\mathrm{f}}\right)$. To calculate a correct system compliance value extrapolation of the data to $(1 / \mathrm{a})=0$ is required. The best measures of the system compliance, $\mathrm{C}_{\mathrm{f}}$ is obtained when the first term of the right is small, that is, for large contact areas [20]. In the TIM machine the system compliance is calculated using the highest load and residual impression. The system compliance is the most important parameter in the elastic modulus calculation. 
The literature indicates that an incorrect value of system compliance is when a plot of Young's modulus against depth of indentation shows deviations on the elastic modulus as function of the depth of penetration this value must be constant. The elastic modulus has depth independent properties. Assuming all other variables involved in the indentation test are correct, and if a large value of system compliance is used, the elastic modulus will be corrected at small radii. However the modulus will steadily decrease at larger radius. If the system compliance is smaller than the correct value a steady increase in the elastic modulus magnitude will be observed [20]. In this research the elastic modulus is plotted against strain. The aforementioned observations are shown in the elastic modulus against strain graph, as seen in Figure 4.22.

To calculate the elastic modulus of the material, the following equations were used in this research. The experimental input values are the contact stiffness from Figure 4.21 and the contact radius from indentation image system:

$$
\begin{gathered}
E^{*}=\frac{S}{2 a} \\
\frac{1}{E^{*}}=\frac{\left(1-v^{2}\right)}{E}+\frac{\left(1-v^{\prime 2}\right)}{E^{\prime}}
\end{gathered}
$$

Figure 4.22 shows a comparison between the calculated linear response and literature values of the elastic behavior of the material. The experimental elastic modulus and the published values of elastic modulus the error is less than 10\% for Aluminum $7075 \mathrm{~T} 6$. 


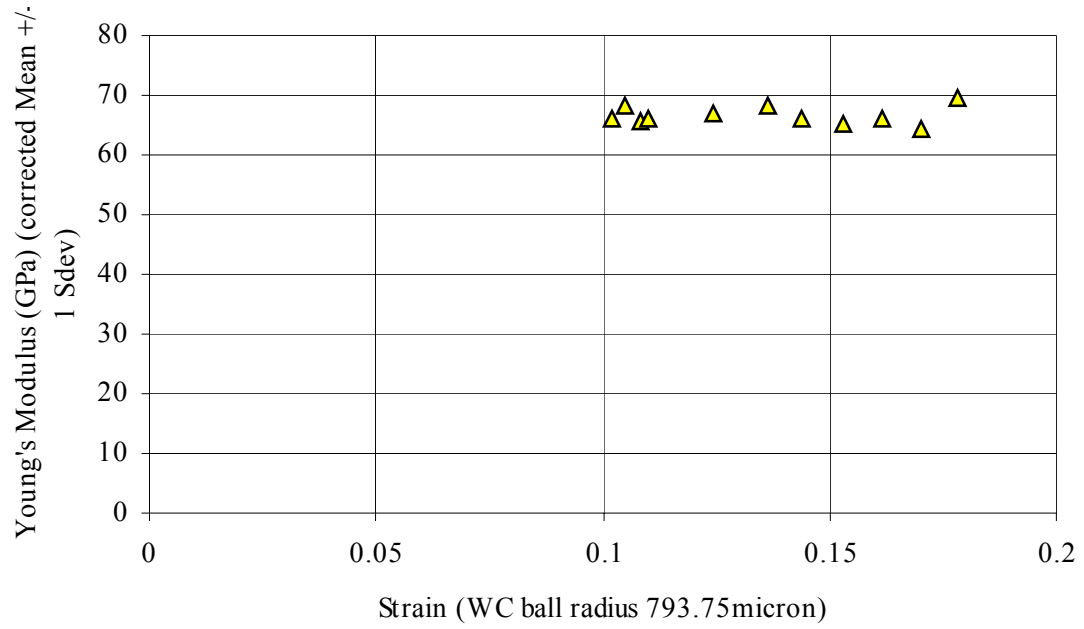

Figure 4.22 Example of elastic modulus by the Depth Sensing Indentation Method

Figure 4.23 is a comparison of the published values and the experimental value for the elastic modulus of Aluminum 7075-T6.

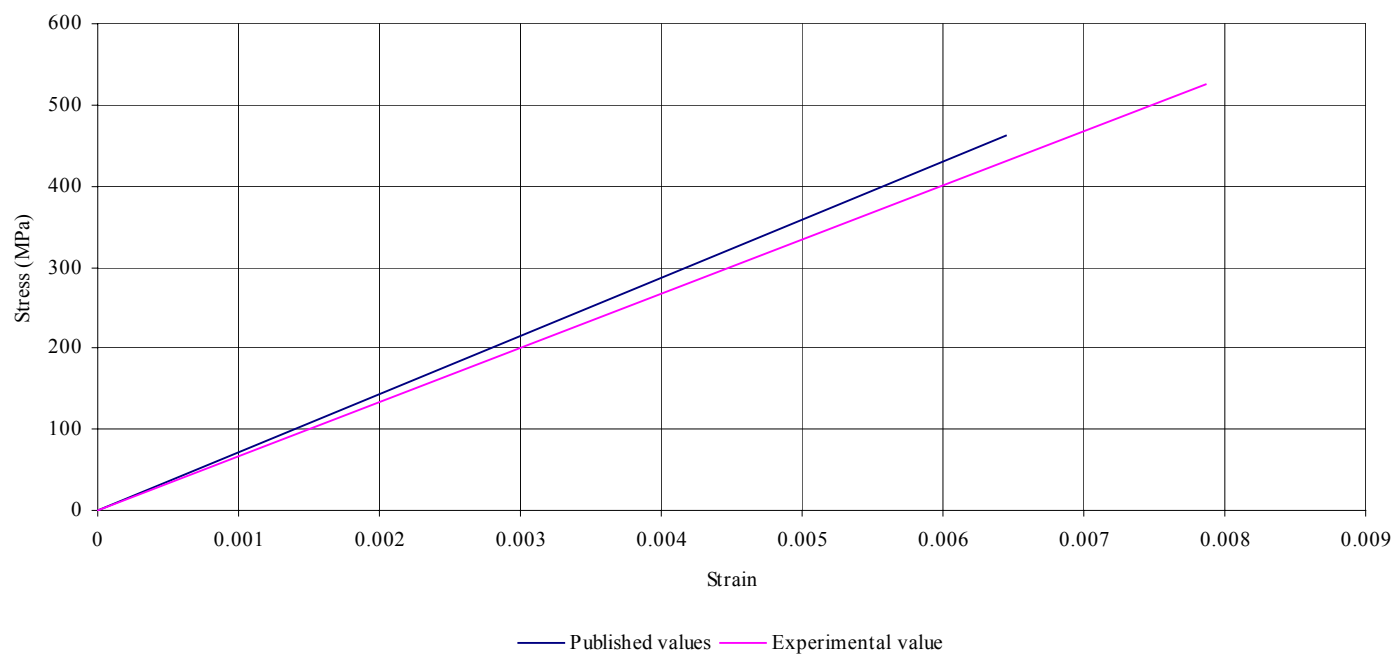

Figure 4.23 The TIM experimental elastic modulus compared with the published value. 


\subsection{Yield strength}

The simple and efficient approach proposed by Konda [21] to determine the yield strength is implemented in this research.

As a first step in Kondas's approach [21] require the calculation of the Meyer's exponent by Equation (4.18). The strain-hardening exponent is derived by Equation (2.29). Meyer's exponent is obtained by plotting the curve of force versus residual impression radius, as shown in Figure 4.24.

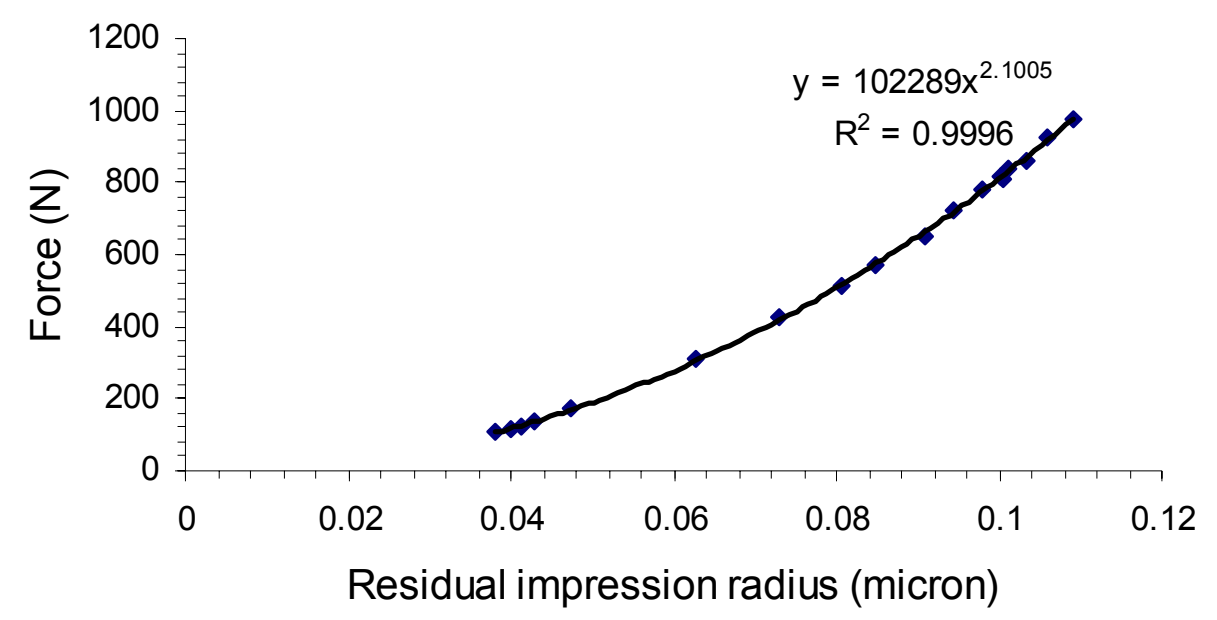

Figure 4.24 Plot of force against residual impression radius used to calculate the Meyer's exponent. Meyer's Law F $=\mathrm{kd}^{\mathrm{m}}$ Material Aluminum alloy 7075-T6

From the residual impression radius and the applied force the indentation stress is calculated with the use of Equation (4.24). To calculate the indentation strain, the residual impression radius is used through Equation (4.19). This is to define the fully plastic region as shown in Figure 4.25. Figure 4.25 shows the results for a relation of residual impression radius/Indenter radius $(\mathrm{a} / \mathrm{R})$ greater than 0.16 as suggested by [18] for the fully plastic region. 
The constraint factor as defined by Tabor [12] is constraint factor defined by Meyer's stress/stress and plays an important role in the yield strength determination by the Konda's approach [21]. A constant value of 2.8 produce an error of about $9 \%$, while a constraint factor dependent on the strain hardening exponent [17], has an error of about $4 \%$ or less in this research.

Figures 4.24 and 4.26 are the representation of the Meyer's equation for Aluminum 7075T6 and Inconel Alloy 783, respectively. These figures are used to determine the Meyer's exponent and then the strain-hardening exponent note the good correlation coefficient for the two graphs.

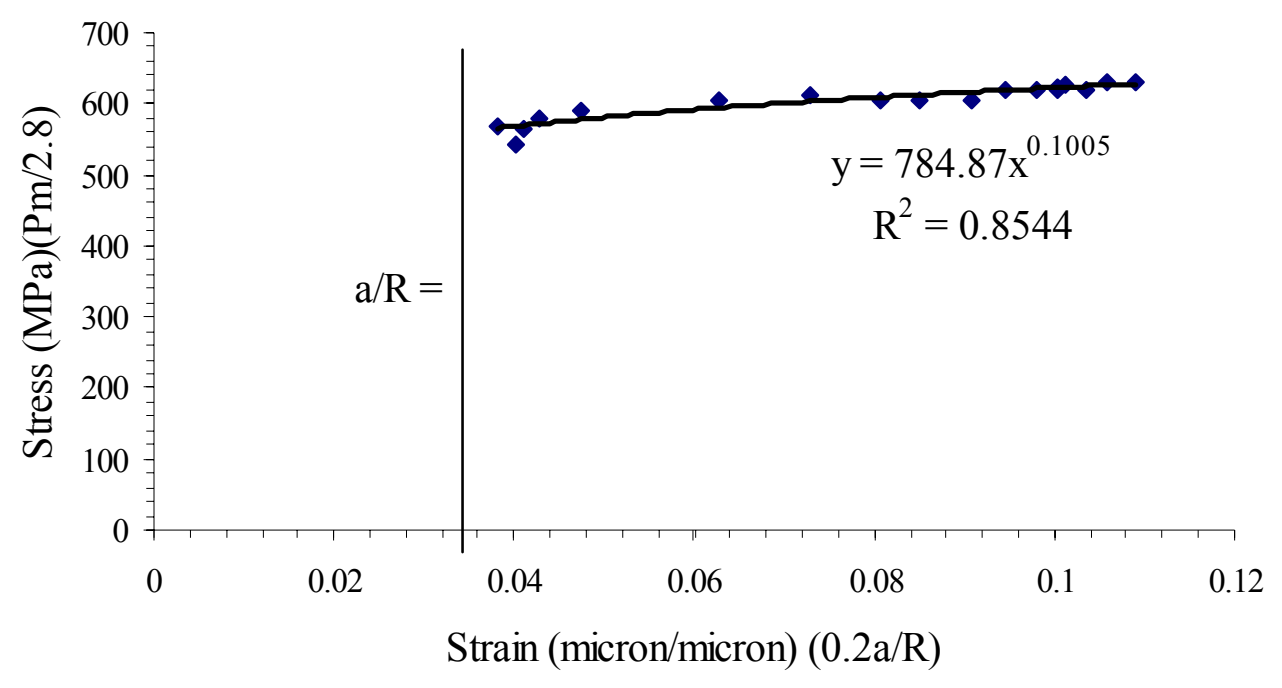

Figure 4.25 Experimental indentation stress - strain for fully plastic region. Material Aluminum 7075-T6. TIM instrument with a sapphire ball indenter of $1.5 \mathrm{~mm}$ diameter.

Figures 4.25 and 4.27 show the results for the fully plastic region. The indentation stress and indentation strain as calculated by the Tabor's equations [12] of stress and strain. For Tabor 
[12] the fully plastic regions start at a value of $\mathrm{a} / \mathrm{R}$ ratio greater than 0.1 . This criterion is used for the Inconel 783 data.

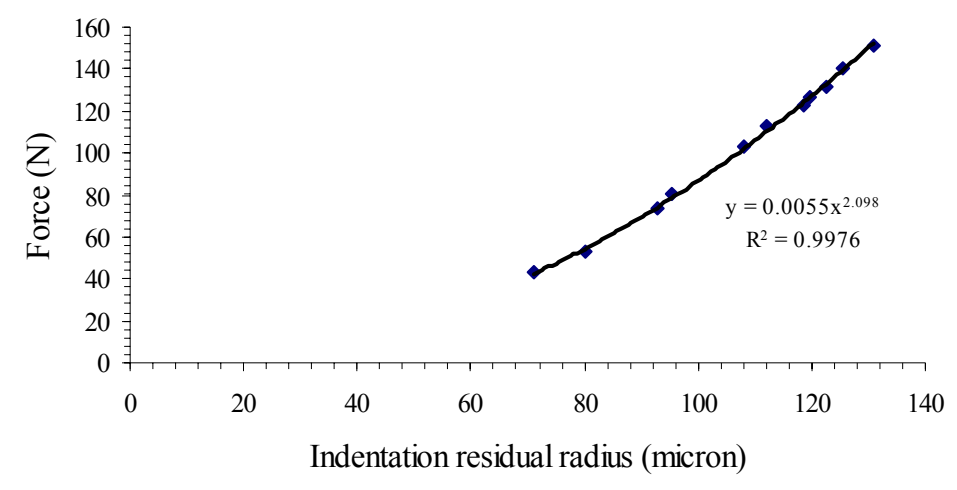

Figure 4. 26 Plot of force against residual impression radius used to calculate the Meyer's exponent. Meyer's Law F = kd $\mathrm{m}^{\mathrm{m}}$ Material Inconel Alloy 783.

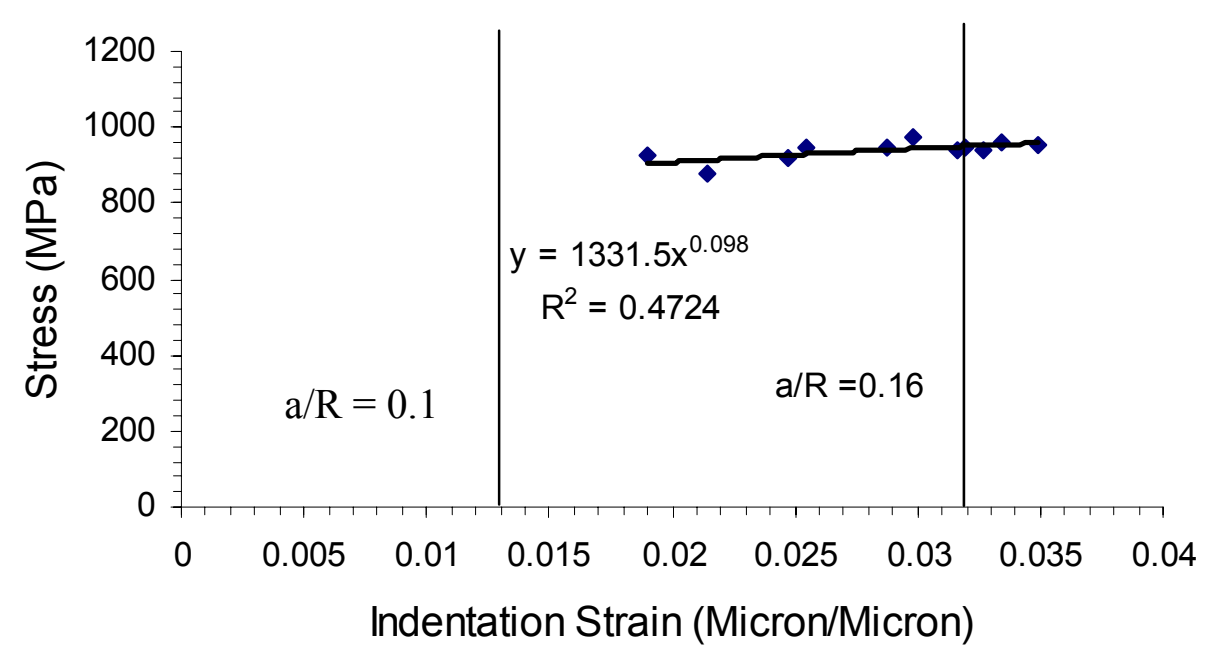

Figure 4.27 Experimental indentation stress - strain. Material Inconel Alloy 783. TIM instrument with a sapphire ball indenter of $1.5 \mathrm{~mm}$ diameter. 


\subsection{The strain-stress curve from the TIM test}

The mechanical parameters of the elastic modulus and the strain-hardening exponent have been experimentally derived for Aluminum Alloy 7075-T6 and Inconel Alloy 783. The Konda's approach [21] is used to calculate the yield strength and it has been implemented with satisfactory results. The mechanical parameter of failure stress cannot be experimentally found in order to define the complete true stress- true strain curve. However with the known values of elastic modulus, yield strength and strain hardening exponent the true stress-true strain curve for Aluminum Alloy 7075-T6 and Inconel Alloy 783 can be generated with accuracy. The indentation experimental curve shows agreement with the literature stress-strain curve. Figure 4.28 represents the true-stress true-strain for Aluminum 7075-T6. Figure 4.29 represents the truestress true-strain curve for Inconel Alloy 783.

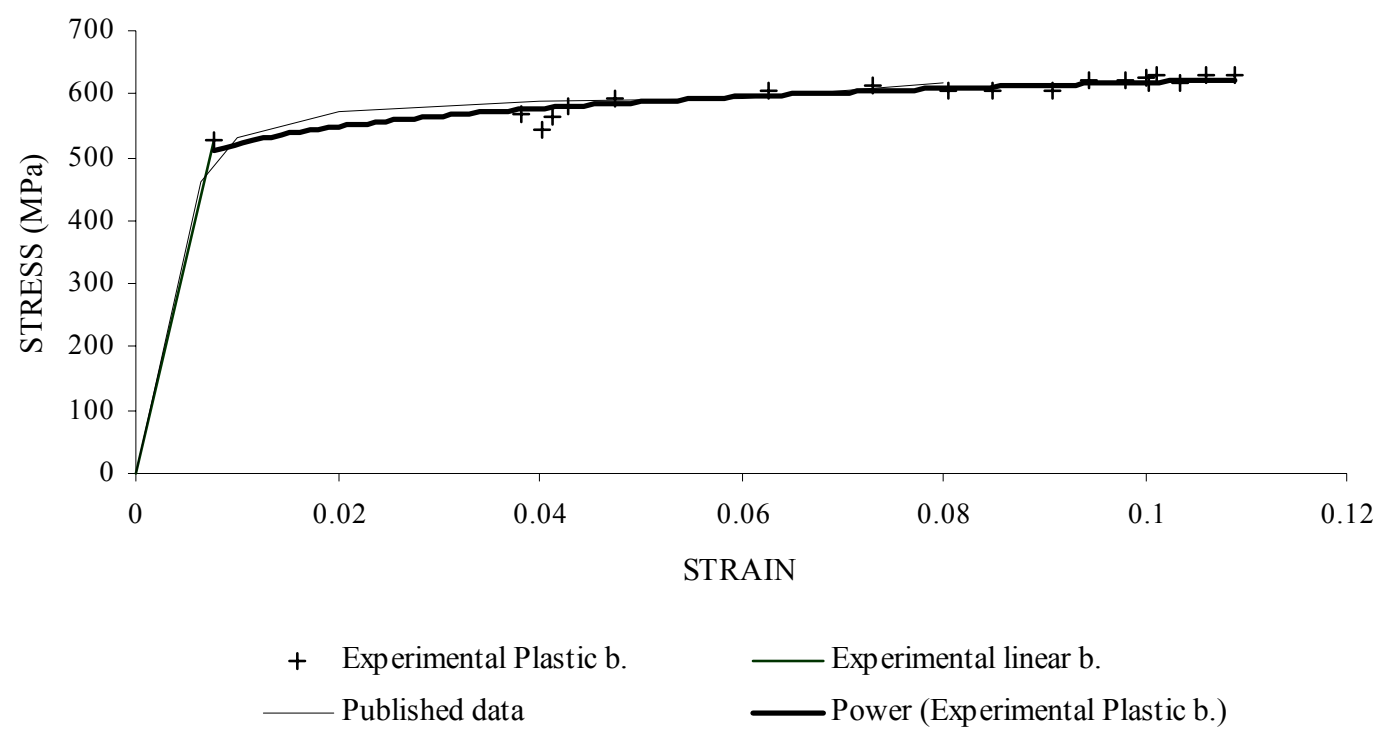

Figure 4.28 Experimental TIM instrument true-stress true strain compared with literature uniaxial true strain true stress curve. Material Aluminum 7075-T6

The yield strength of the material is the intersection of the elastic curve and plastic curve fitting. The error on the Yield strength is less than 10\%. [21] 


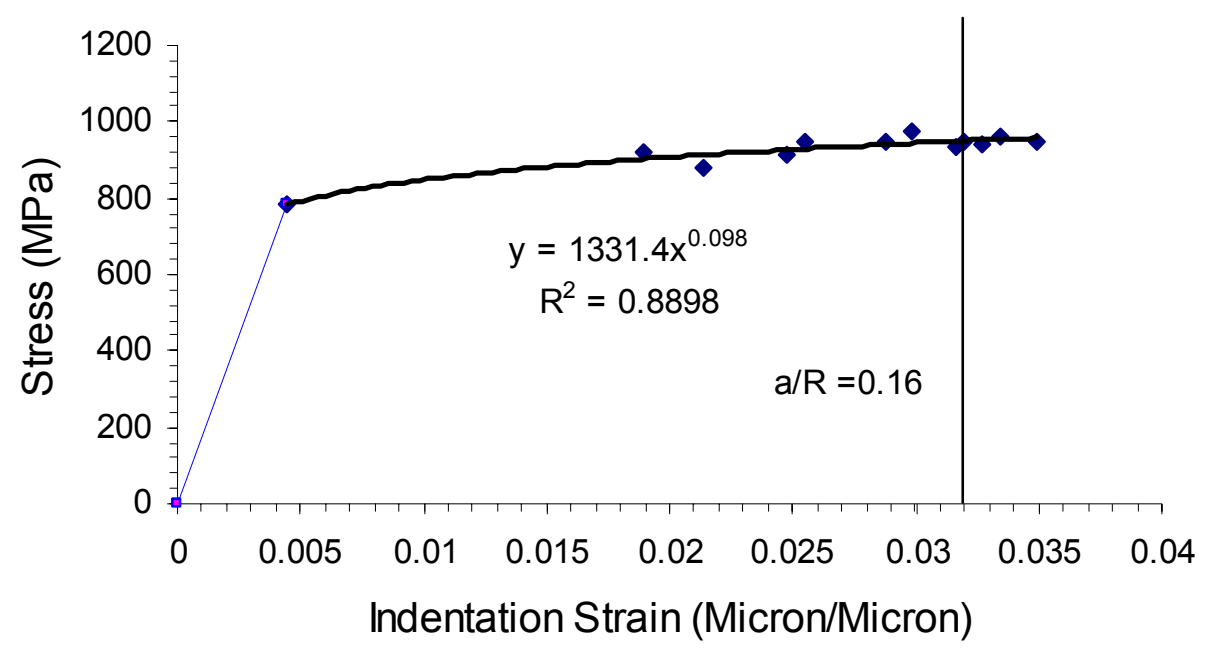

Figure 4.29 Experimental TIM instrument true-stress true strain curve for Inconel Alloy 783

The following table summarizes the characteristics of the values of the mechanical properties obtained by the TIM instrument and the optical Newton's ring method.

Table 4. 3 Mechanical Properties by the optical method-Newton rings.

\begin{tabular}{|c|c|c|c|c|}
\hline Material & $\begin{array}{c}\text { Mechanical } \\
\text { Properties }\end{array}$ & Average & $\begin{array}{c}\text { Standard } \\
\text { Deviation }\end{array}$ & $\begin{array}{c}\text { Error } \\
\text { Percent } \\
\text { Maximum } \\
\text { \% }\end{array}$ \\
\hline $\begin{array}{c}\text { Aluminum } \\
\text { Alloy 7075-t6 }\end{array}$ & $\begin{array}{c}\text { Elastic modulus } \\
\text { GPa }\end{array}$ & 72.192 & 1.72 & 4 \\
\cline { 2 - 5 } & $\begin{array}{c}\text { Yield strength } \\
\text { Mpa }\end{array}$ & 474.64 & & 2.15 \\
\hline $\begin{array}{c}\text { Inconel } \\
\text { Alloy 783 }\end{array}$ & $\begin{array}{c}\text { Elastic modulus } \\
\text { GPa }\end{array}$ & 178.054 & 5.253 & 4.56 \\
\cline { 2 - 5 } & $\begin{array}{c}\text { Yield strength } \\
\text { MPa }\end{array}$ & 782.90 & & 0.38 \\
\hline \multirow{2}{*}{$\begin{array}{c}\text { Molybdenum } \\
\text { Alloy 695 }\end{array}$} & $\begin{array}{c}\text { Elastic Modulus } \\
\text { GPa }\end{array}$ & 138.66 & & 43.20 \\
\cline { 2 - 5 } & $\begin{array}{c}\text { Fracture Force } \\
\text { Newton }\end{array}$ & 15 & & \\
\hline
\end{tabular}




\section{Chapter 5 SUMMARY AND FUTURE WORK}

\subsection{Summary}

This dissertation involves two main tasks. First is the development of a TIM experimental to obtain material stress/strain curve or mechanical properties. Second involve the conceptual design, manufacture, and assembly of a TIM prototype and software.

A new method to calculate the elastic modulus with the use of simplified TIM instrument was developed. The method uses the Newton's rings generated during the indentation at low loads to obtain the material elastic modulus. Good agreement between literature values of Aluminum 7075-T6 and Inconel 783 show the validity of the TIM-Newton ring method for material elastic modulus determination.

The simplified TIM instrument has the capability to calculate the elastic modulus by Oliver and Pharr's method, as an instrumented indentation test.

The TIM instrument provides the force and the residual impression radius that allows analysis in the fully plastic region. The indentation stress is deduced from the Meyer's stress and the strain is calculated by the Tabor's equations [12]. The contact radius and force are also used to obtain the strain-hardening factor using the Meyer's equation. With the strain hardness factor and the elastic modulus previously calculated, the yield strength could be found by the use of Konda's approach [21].

Finally with the elastic modulus, yield strength and strain-hardening exponent known, the true-stress strain curve can be generated. 


\subsection{Recommendations and future perspectives}

Future studies are needed to improve the simplified portable TIM instrument. It is necessary to conduct more tests to evaluate the elastic modulus of other materials. Conduct more tests with the specific goal of determine the system compliance for each configuration of load cell and indenter when the Oliver and Pharr method is used. In this manner the robustness of the simplified TIM instrument can be verified with other materials like ceramics.

The measurements of the Newton rings' radii were done manually. A more sophisticated and automatic image analysis is recommended in order to optimize the data acquisition process and analysis.

Sapphire is the indenter material used in the present simplified TIM configuration. Sapphire has the advantage of having perfect spherical shapes that can be manufactured. For hard metals and ceramics however, the use of a diamond indenter is recommended.

The actual simplified TIM instrument operates under displacement control. Function under load control will improve the applications of the TIM instrument, e.g. fatigue test and fracture toughness determination in ceramics.

The mechanism of the simplified TIM instrument must be improved with the experience obtained from the use of the equipment. For example, the stiffness of the positioning control mechanism can be increased by provided more area of support. The microscope positioning mechanism can be software-controlled for letter image focusing. 


\section{REFERENCES}

1. ASTM. Standard Test Methods for Tension Testing of Metallic material. Designation E804. Annual book of ASTM Standards Volume 03.01. 1993.

2. ASM International, Edited by Howard E. Boyer. Hardness Testing. ASM INTERNATIONAL, Metals Park, OH 44073.

3. ASTM. Standard Test Methods for Brinell Hardness of Metallic Materials has the designation E-10-xx. Volume 03.01 Metals - Mechanical testing; Elevated and LowTemperature tests; Metallography. ASTM. 1993

4. ASTM. Test Method for Microhardness of Materials, E 384-xx. Volume 03.01 Metals Mechanical testing; Elevated and Low-Temperature tests; Metallography. ASTM. 1993

5. ASTM. Test methods for Young's Modulus, Tangent Modulus and chord Modulus. Designation E 111-xx Volume 03.01 Metals - Mechanical testing; Elevated and LowTemperature tests; Metallography. ASTM. 1993

6. ASTM. Terminology Related to Methods of Mechanical Testing. Designation E-7-xx. Volume 03.01 Metals - Mechanical testing; Elevated and Low-Temperature tests; Metallography. ASTM. 1993.

7. Fisher-Cripps, Anthony C. Introduction to Contact Mechanics. Springer. 2000.

8. Lawn, Brian R. Indentation of Ceramics with Spheres: A Century after hertz. J. Am. Ceramics. Soc. 81, pp 1977-1993. 1988.

9. McCann, Martha Mary. Nanoindentation of Gold Single Crystals. PhD. Dissertation. Blacksburg, Virginia. 2004.

10. http://www.loc.gov/catdir/samples/wiley032/2002155484.pdf.

11. http://metalrg.iisc.ernet.in/ workshop/Talk2-Sundararajan.pdf

12. Tabor, David, The hardness of Metals. Oxford. 1951.

13. Oliver, W.C., Pharr, G.M. An improved technique for determining hardness and elastic modulus using load and displacement sensing indentation experiments. J. Mater. Res., Vol. 7, No 6, pp. 1564-1583. 1992.

14. Mesarovic, S. and Fleck N. Spherical indentation of elastic-plastic solid. Proc. Royal Soc. Vol. 455, pp 2707-2728. 1999.

15. Beghini, M. Bertini, L. Fontanari, V. Evaluation of the flow curve of metallic materials by means of spherical indentation, in Computational Methods in Contact Mechanics, Vol. 1, pp. 241-252. 2001.

16. Taljat, B., Zacharia, T. and Haggag, F.M. Analysis of ball-indentation load depth data: Part I, Determining elastic Modulus. J. Mater. Res. Vol. 12, No.4, pp 965-974. 1997.

17. Taljat, B. Zacharia, T. Kosel, F. New analytical procedure to determine stress-strain curve from spherical indentation data. Int. J. Solids Structures Vol 35 pp 4411-4426, 1998.

18. Herbert, E.G., Pharr, G.M., Oliver, W.C., Lucas, B.N. and Hay, J.L. On the measurement of stress-strain curves by spherical indentation. Thin Solid Films, pp 398-399. 2001.

19. Standard Test Method for Automated Ball Indentation testing of Metallic Samples and structures to determine Stress-strain Curves and Ductility at Various Test Temperatures. Draft 12. www.atc-ssm.com. 
20. Hay, J.L. Pharr, G.M. Instrumented Indentation Testing. ASM International, Vol. 3, Issue 6, 2003.

21. Konda, Bharath. Methodology to Determine Post Yield Material Mechanical Properties from Spherical Indentation. Master Thesis. Department of Mechanical and Aerospace Engineering. WV, 2005.

22. Metallic materials - Instrumented indentation test for hardness and materials parameters. Part 1. Test method. ISO 14577-1:2002.

23. Stanbury, G.C., Davis, F.A. UK's Provision of Primary Hardness Standards. National Physical Laboratory, Teddington, Middlessex. UK. Proc. XVI IMEKO World Congress, Vienna, Austria, 2000.

24. NIST Develops New National Hardness Standards to Help Increase U.S. Trade. www.nist.gov/director/states/ma/fy03 ma 17.htm.

25. Hardness Testing Information Guide. Part 3. www.hardnesstesters.com.

26. Common Problems in Microhardness Testing. www.hardnesstesters.com.

27. Hay, J.L., Pharr, G.M. Instrumented Indentation Tests. ASM Handbook volume 08.

28. http://www.nanoindentation.cornell.edu/Machine/Nanoindentation-Machine.htm

29. Feng, Chuanyu. Evaluation of Material Mechanical Properties Using Depth Sensing Indentation and Combined Twyman-Green Interferometry and Moire Interferometry. Ph.D. Dissertation. Department of Mechanical and Aerospace Engineering. West Virginia University. 2005.

30. www.citycollegiate.com/newtons rings.htm

31. Johnson, K. L., Contact Mechanics. Cambridge University Press. 1985.

32. Wolf, B., Inference of Mechanical Properties from Instrumented Depth Sensing Indentation at Tiny Loads and Indentation Depths. Cryst. Res. Technol. Vol. 35, pp 377-399. 2000.

33. www.internationalcrystal.net

34. www.matweb.com

35. Inconel alloy 783. Special metals. www.specialmetals.com

36. Sun, DongXiang. Thermomechanical Properties of Molybdenum Alloys with Dispersing $\mathrm{MgAl}_{2} \mathrm{O}_{4}$ (Spinel) and MgO Particle. Master Thesis, WVU. 2003.

37. Yao, Ziheng. Development of an Indentation Method for Material Surface Mechanical Properties Measurements, PhD Dissertation, West Virginia University. 2005. 


\section{APPENDIX A YIELD STRENGHT}

The simplified TIM instrument produces the load-depth curve that represents a cycle of indentation, load and unload. The loading slope is calculated and compared with the unloading slope in a ratio. A plot of loading slope/unloading slope ratio against the applied load can be used to determine the yield strength of a material. Figure A.1 shows the loading slope /unloading slope ratio against the applied force, and clearly shows the three regions of elastic, elastic-plastic and fully plastic regions. The results are for Aluminum 7075-T6. The data used in Figure A.1 is not corrected for system compliance. If the data were corrected for system compliance will shows more abrupt changes. Additional test are need to produce data in which the system compliance can be determined and apply this correction to the data to obtain results without any complain. The corrected data will show more accurate trends to determine the yield strength by this method.

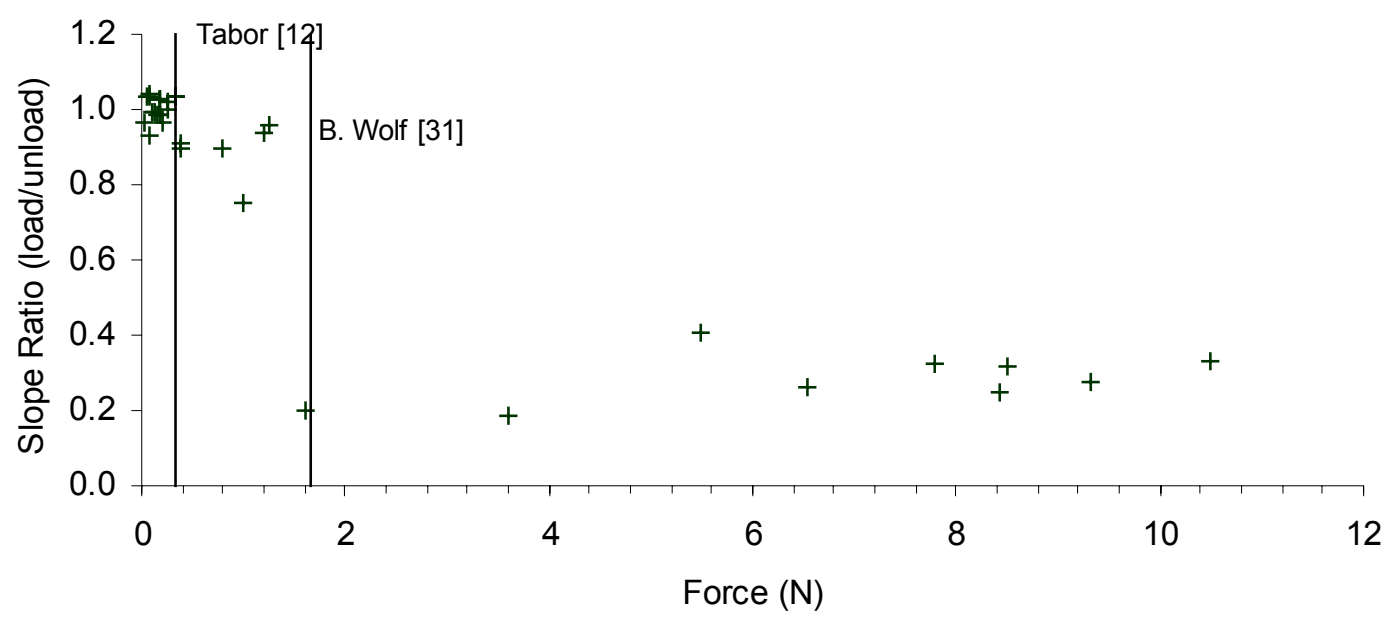

Figure A. 1 Loading Slope / Unloading slope against force. Aluminum 7075-T6

Figure A.2 and A.3 show a graph loading slope stage of the cycle against applied load. Interesting preliminary results are observed. At a load of less than $0.32 \mathrm{~N}$, i.e. the limit load for 
the Hertz predicted elastic range; the data has a mostly constant slope. If the load increases a change in slope is observed, the region where the plastic response start to be developed. After a load of 1.6 N, an abrupt change in slope is seen. This corresponds to the elastic-plastic region. After this region, the slope relates to the fully plastic region. Figure 5.3, shows the data for a load of up to $2 \mathrm{~N}$., the behavior discussed above is thus observed.

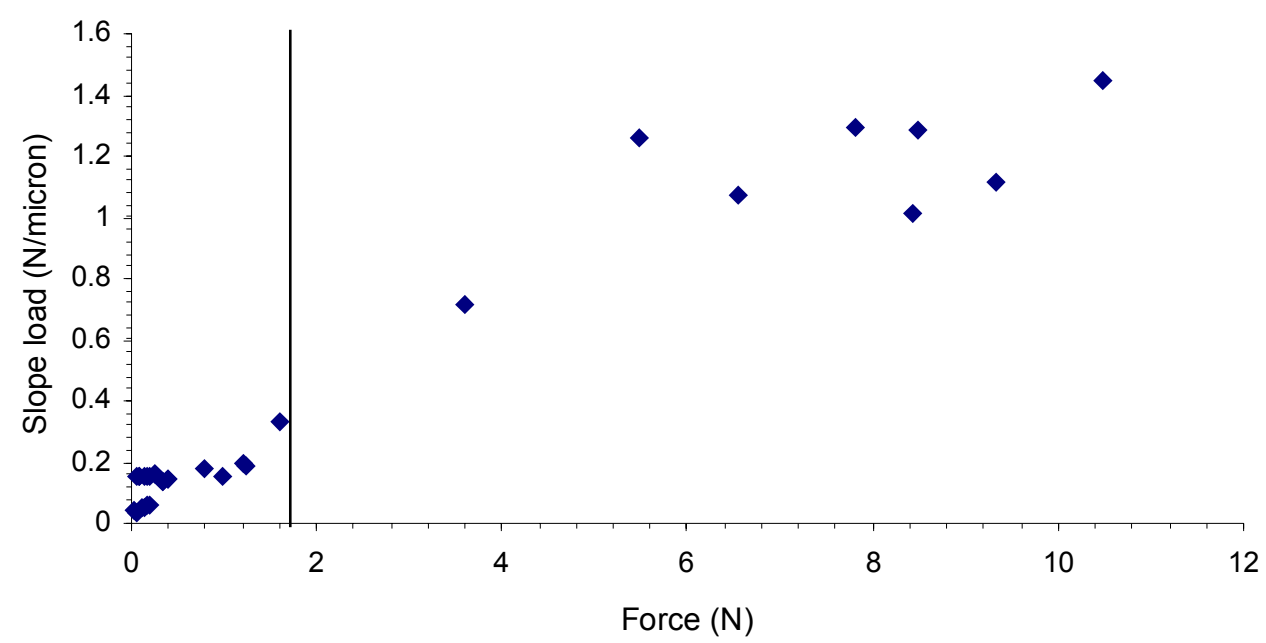

Figure A. 2 Loading slope portion of the load-piezoelectric displacement curve against force. Aluminum 7075-T6 
Aluminum 7075-T6

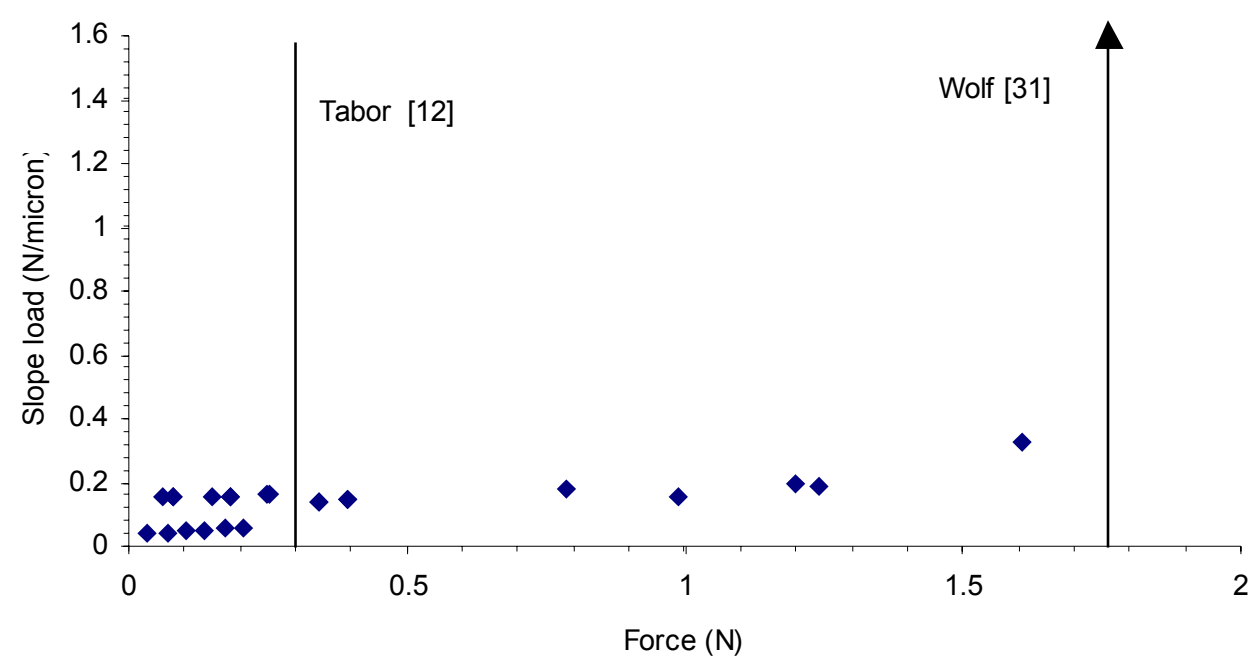

Figure A. 3 Loading slope portion of the load-piezoelectric displacement curve against load. The load is shown until $2.0 \mathrm{~N}$. Aluminum 7075-T6 


\section{APPENDIX B APPLICATION TO BRITTLE MATERIALS}

The simplified TIM instrument has a promissory future in the area of brittle materials. To illustrate the TIM capacity to determine the load at which the material fails by cracks Figure B.1 and pictures in Figure B.2 are presented .The data was obtained by the sapphire indenter, but this data is not conclusive due to similar elastic modulus with the pure molybdenum and sapphire indenter, which induces errors in the measurement.

For Molybdenum Alloy 695 Figures B.2, B.3 and B.4 are presented. The same comments apply for these graphs that for the behavior in Aluminum alloy 7075-T6 in Appendix A.

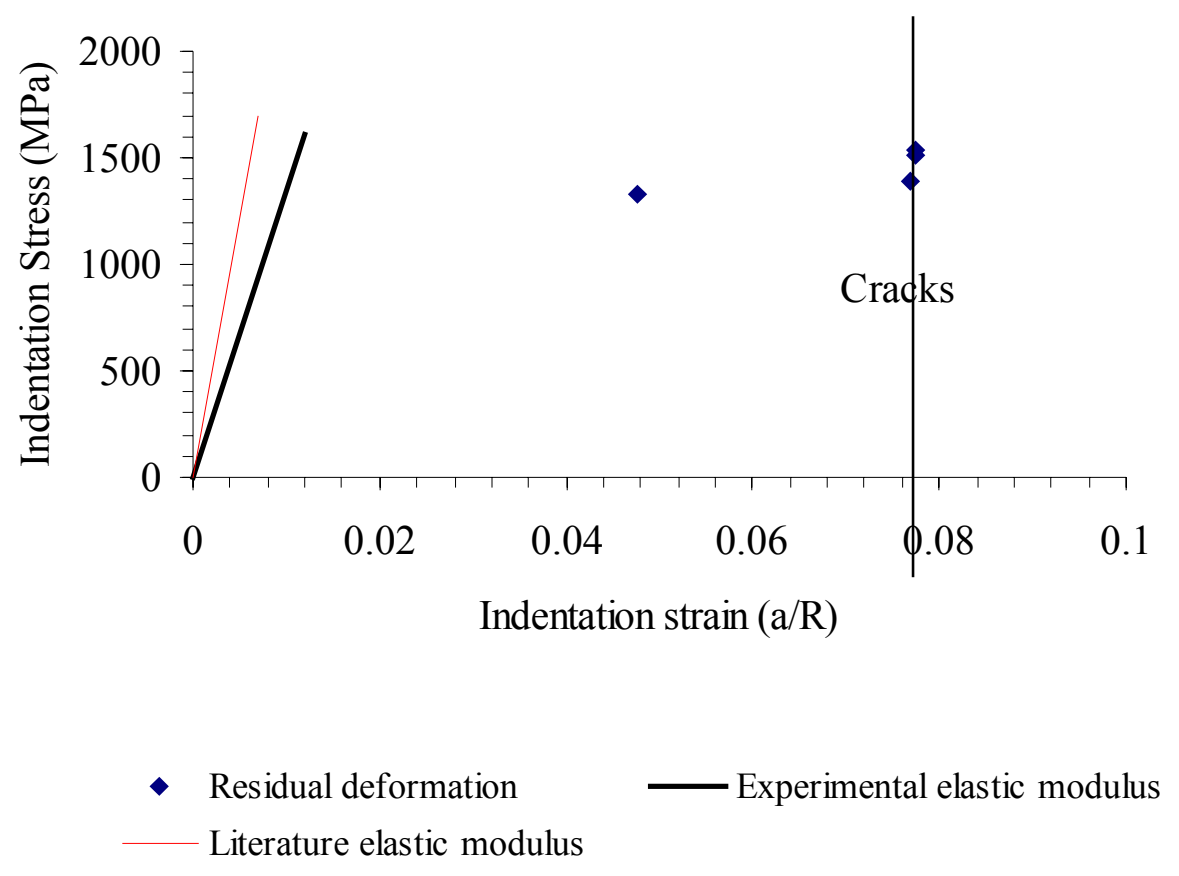

Figure B. 1 Simplified TIM results for Molybdenum alloy 695. 


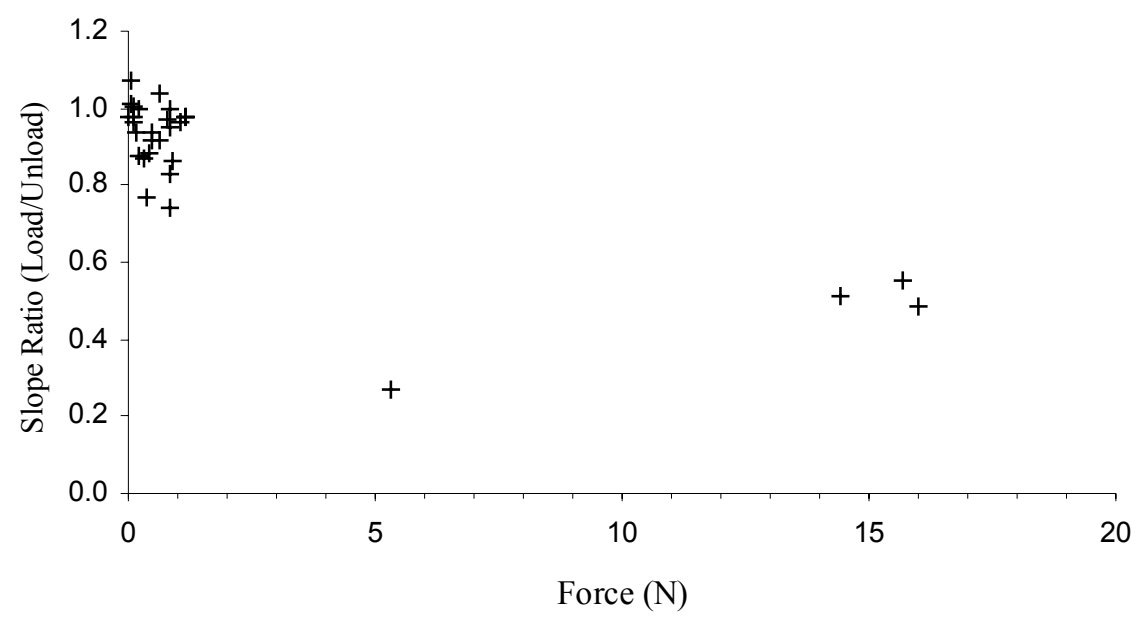

Figure B. 2 Load-piezoelectric displacement curve unloading slope/loading slope versus Force for Molybdenum Alloy 695.

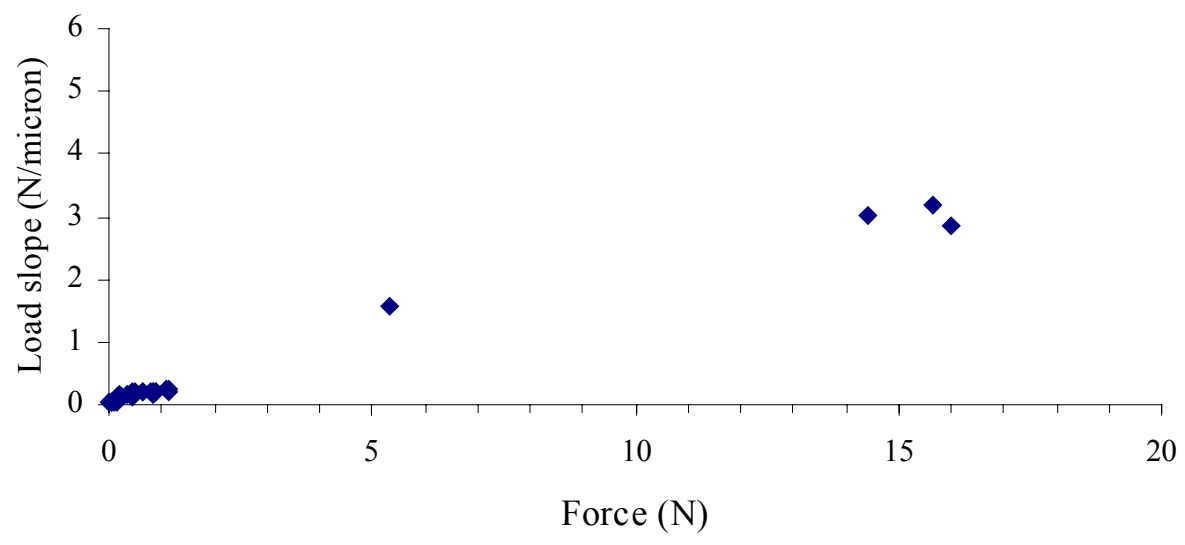

Figure B. 3 Loading slope of the load-piezoelectric displacement curve against force. Molybdenum Alloy 695. 


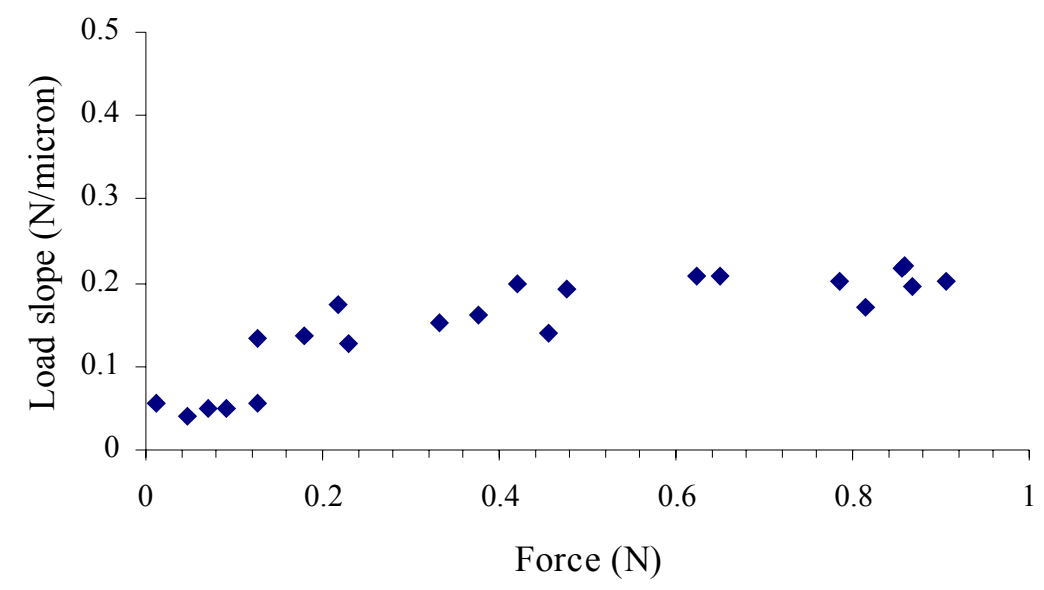

Figure B. 4 Loading slope portion of the load-piezoelectric displacement curve. The load is shown until $2.0 \mathrm{~N}$. Aluminum 7075-T6

Figure B.5 show the pictures for Molybdenum Alloy 695. Image a) show clearly a residual indentation, without crack, pictures a), b) and c) shows the crack at a force near $15 \mathrm{~N}$. 


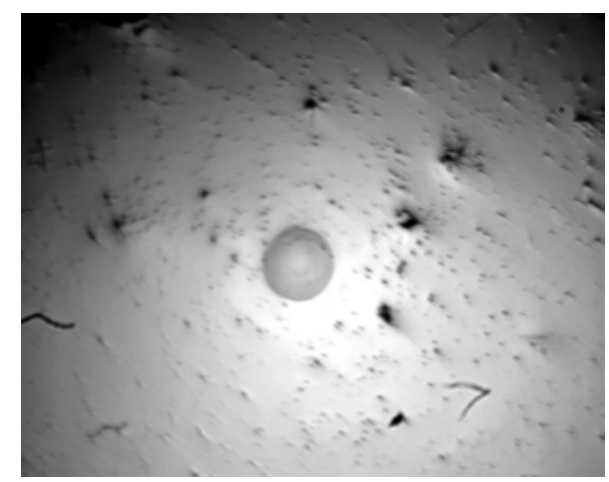

(a)

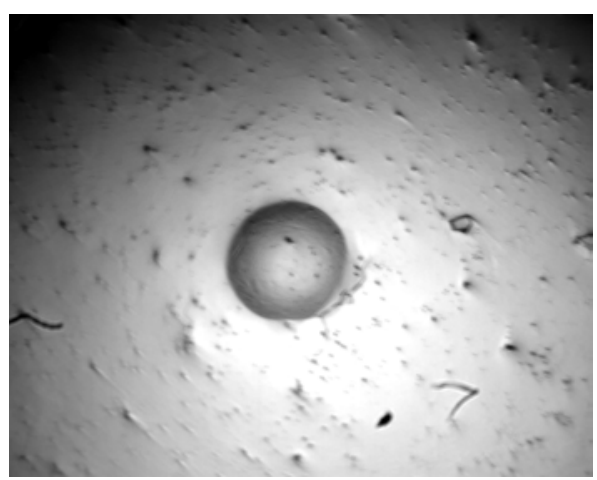

(c)

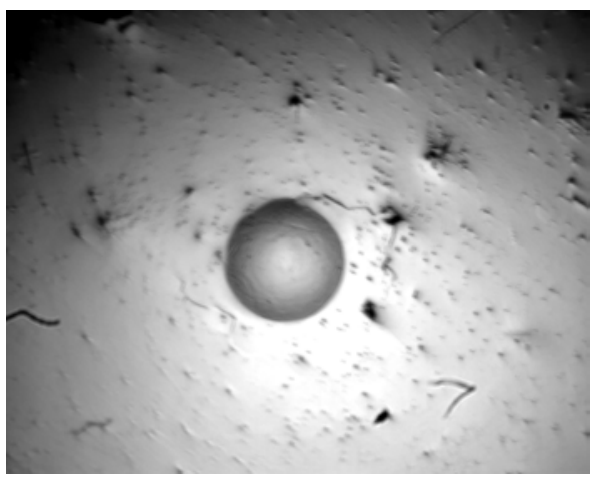

(b)

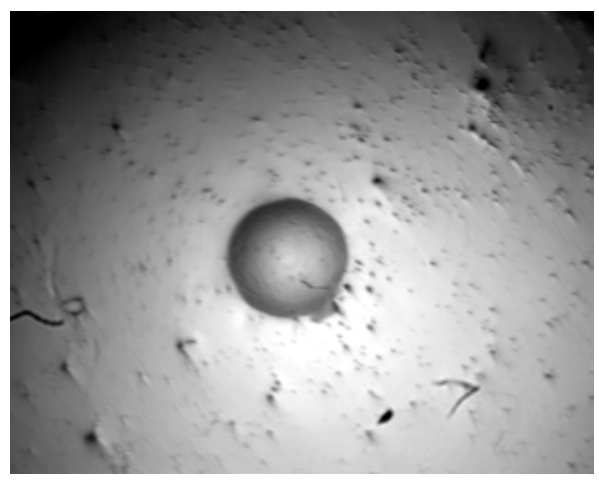

(d)

Figure B. 5 Simplified TIM instrument pictures for Molybdenum Alloy 695.

The simplified TIM instrument can record the image and force at the time when materials fail. The image provides the radius of indentation, crack length, crack location. The data provided by the TIM instrument can be used to calculate the elastic modulus of the materials and the fracture toughness of the brittle materials. 